\title{
Investigation of the segregated visual system
}

\author{
Ph. D. Thesis
}

Gergő Csete M.D

Szeged

2016 


\title{
Investigation of the segregated visual system
}

\author{
$\mathrm{PhD}$ Thesis \\ Gergő Csete, M.D.
}

Clinical and Experimental Neuroscience Program,

Doctoral School of Clinical Medicine, Faculty of Medicine, University of Szeged

Supervisor: Zsigmond Tamás Kincses, M.D., Ph.D., Habil. Associate Professor Department of Neurology, Albert Szent-Györgyi Clinical Center, University of Szeged 


\section{Table of contents}

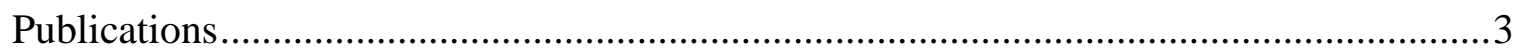

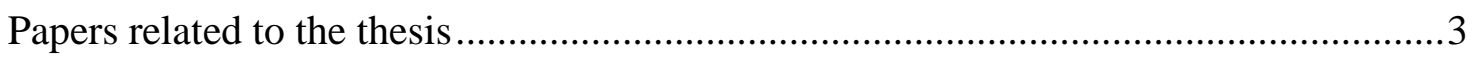

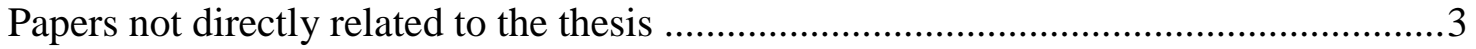

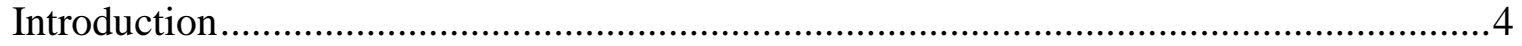

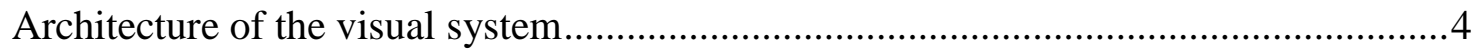

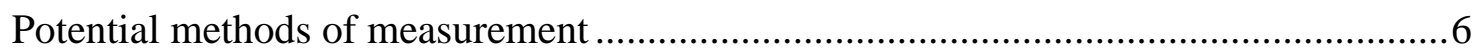

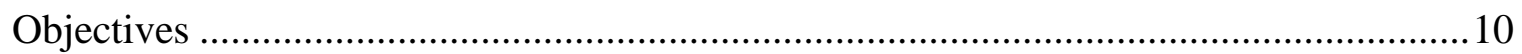

Aging alters visual processing of objects and shapes in inferotemporal cortex in monkeys

Methods .12

Results

An investigation of the white matter microstructure in motion detection using diffusion MRI

Methods .19

Results .22

Audio-visual integration through the parallel visual pathways .24

Methods .25

Results .28

Discussion .31

Aging effects on object vision in monkeys . .31

Attention modulation of motion perception... .34

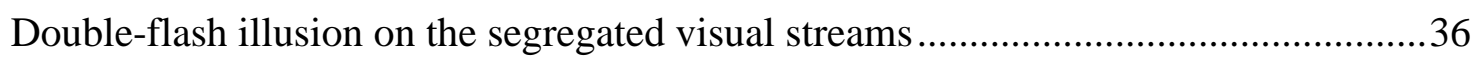

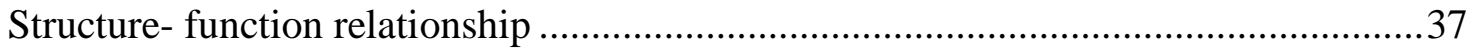

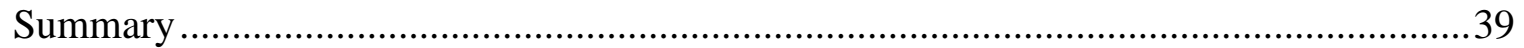

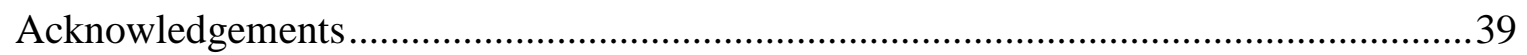

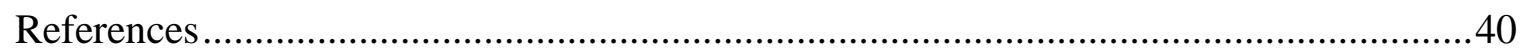

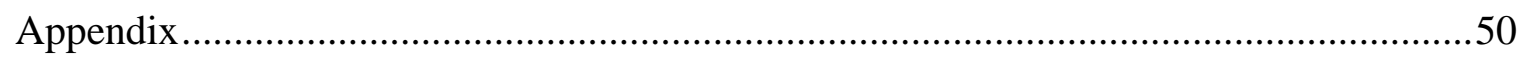

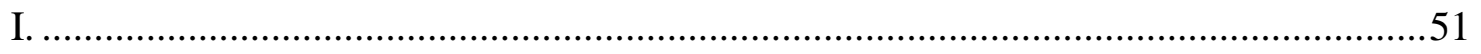

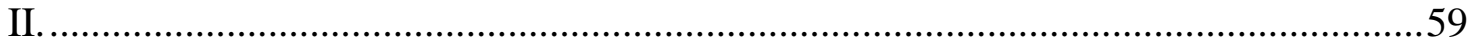

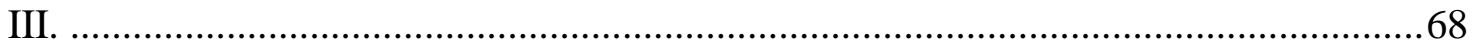




\section{$\underline{\text { Publications }}$}

\section{Papers related to the thesis}

I. Kaposvari P , Csete G, Bognar A , Csibri P, Toth E, Szabo N , Vecsei L, Sary G, Tamas Kincses Z

Audio-visual integration through the parallel visual pathways.

BRAIN RESEARCH 1624: pp. 71-77. (2015), IF: 2.561

II. Csete G, Bognar A, Csibri P, Kaposvari P, Sary G

Aging alters visual processing of objects and shapes in inferotemporal cortex in monkeys.

BRAIN RESEARCH BULLETIN 110C: pp. 76-83. (2015), IF: 2.572

III. Csete G, Szabo N , Rokszin A, Toth E, Braunitzer G, Benedek G, Vecsei L, Tamas Kincses $\mathrm{Z}$

An investigation of the white matter microstructure in motion detection using diffusion MRI.

BRAIN RESEARCH 1570: pp. 35-42. (2014), IF: 2.843

\section{Papers not directly related to the thesis}

I. Kiraly A, Szabo N, Toth E, Csete G, Farago P, Kocsis K, Must A, Vecsei L, Kincses ZT.

Male brain ages faster: the age and gender dependence of subcortical volumes

BRAIN IMAGING BEHAV. 2016 Sep;10(3):901-10, IF: 3.667

II. Kincses ZT, Toth E, Banko N, Vereb D , Szabo N , Csete G , Farago P , Kiraly A, Bencsik K, Vecsei L

Grey matter atrophy in patients suffering from multiple sclerosis

IDEGGYÓGYÁSZATI SZEMLE / CLINICAL NEUROSCIENCE 67:(9-10) pp. 293-300. (2014), IF: 0.386

III. Kincses ZT , Horinek D , Szabo N , Toth E, Csete G, Stepan-Buksakowska I, Hort J , Vecsei L

The pattern of diffusion parameter changes in Alzheimer's disease, identified by means of linked independent component analysis.

JOURNAL OF ALZHEIMERS DISEASE 36:(1) pp. 119-128. (2013), IF: 3.612

IV. Szabo N , Kincses ZT , Pardutz A, Toth E, Szok D , Csete G, Vecsei L

White matter disintegration in cluster headache.

JOURNAL OF HEADACHE AND PAIN 14:(1) Paper 64. 6 p. (2013), IF: 3.281

V. Kaposvári P, Csibri P, Csete G, Tompa T, Sáry G

Auditory modulation of the inferior temporal cortex neurons in rhesus monkey

PHYSIOLOGICAL RESEARCH 60:(Suppl. 1) pp. S93-S99. (2011), IF: 1.555 


\section{$\underline{\text { Introduction }}$}

Most of our information from the outer world is gained by vision. An intact visual system is crucial for life and for the optimal recognition. For correct explanation and interpretation of discrepancies caused by certain diseases, the thorough knowledge of the visual system is substantial. It also helps to understand the human development better and helps to identify improved biomarkers for diagnosis and treatment. For the facilitation of these opportunities, it is important to investigate the whole system from the neural level to the global, the network level.

\section{Architecture of the visual system}

The light after reflection from the objects passes through the lens and cornea to the retina where the fototransduction takes place. After the primary processing, the information of the ganglion cells is sent to the lateral geniculate body (LGB). Three functionally distinct subtypes of ganglion cells, such as the magnocellular (M), the parvocellular (P) (and koniocellular $(\mathrm{K})^{1}$ ) neurons are located here. M-cells respond to moving stimuli, speed and location in space and show great sensitivity to low contrast. On the other hand, Pcells prefer colours, shape and higher spatial frequencies (Skottun, 2015). The fibres project to the calcarine sulcus via the optic radiation. In the occipital pole, the information from the retina is mapped point by point. Fibres from the retina keep their relative positions to each other, serving retinotopic organization. The axon bundles from the parvocellular and magnocellular system remain separated at this level and reach different layers of the primary visual cortex. The occipital cortex has a large amount of internal granular cells with myelinated axons that cause a distinctive stripe in the cerebral cortex that are called lines of Gennari (Gennari, 1782). V1 is built by orientation columns, "blob"-s and ocular dominance columns. All together it is a virtual neuron-cube, called hypercolumn (Hubel and Wiesel, 1968), that dissociates the detected image on the retina into small building elements, or features (e.g. lines, colours, orientation).

From the primary visual cortex the visual information is passing through two major pathways, to the parietal lobe and to the temporal lobe: the so-called dorsal and ventral pathways, respectively, traditionally regarded as the 'where' and the 'what' stream

\footnotetext{
${ }^{1}$ The koniocellular system is not the main target of our investigations; hence no further details are given.
} 
(Haxby et al., 1991; Ungerleider and Haxby, 1994). Two relevant processing stages should be mentioned as presumably final unimodal stages of the segregated streams as the middle-temporal (MT) and the inferior-temporal cortex (IT).

The region MT is sensitive to movement, depth and activated during visuo-spatial tasks. Higher levels of perception, such as global motion recognition seems to be located in the middle temporal area (MT), medial superior temporal cortex (MST), and the fundus of the superior temporal cortex (FST), that share similar features in monkeys and humans (Morrone et al., 2000), and was suggested to make up a complex: V5/MT+ (Boussaoud et al., 1990; Morrone et al., 2000). This area receives direct input from the primary visual cortex (V1) (Felleman and Van Essen, 1991; Maunsell and van Essen, 1983), LGN (Sincich et al., 2004), and also extrastriate regions, such as V2 (Lewis and Van Essen, 2000). These neurons with a broadly binocular representation and a relatively large receptive field (( $15-20^{\circ}$, (Angelucci et al., 2002), have a principal role in motion and directional sensitivity (Chawla et al., 1998).

The IT is the last unimodal area in the ventral visual stream (Ungerleider, 1982) which receives the visual input mostly from areas V4 and the posterior part of the IT (TEO,(Baleydier and Morel, 1992; Merigan, 1996; Morel and Bullier, 1990). Receptive field of these cells span about $50-60^{\circ}$ and contains binocular representation of the sighted world. Cells in the IT respond best to complex, colourful stimuli (Desimone et al., 1984). They might be selective to shapes, i.e., they respond to, or rather respond more strongly to some shapes than to others (Desimone et al., 1984; Gross et al., 1972; Tanaka et al., 1991) and show strong invariance against certain modifications (Sáry et al, 1993). The IT is closely associated to perception. Micro-stimulation of the area influences the face nonface categorization (Afraz et al., 2006), and human perception models show similar cell firing patterns as IT neurons (Allred et al., 2005). Furthermore, the use of the binocular rivalry paradigm (Leopold and Logothetis, 1996; Sheinberg and Logothetis, 1997) proves that visual perception and IT activity are closely related. 


\section{Potential methods of measurement}

The brain is an integrating different functions, such as perception, attention, learning or execution, etc. Various regions show activity during different tasks, but these together make the basis of our perceptions, thinking or simply, existence. One of the first physiological evidence of brain machinery originates from more than a century ago, when an Italian researcher discovered that frontal brain pulsation is elevated during mental arithmetic task (Mosso, 1881). Currently, many different tools are available to study the central nervous system from the molecular scale to the whole brain networks. All of them have many advantages and also disadvantages. The main differences are spatial and temporal resolution, the origin of the signal and the sensitivity and specificity for the inspected signal. In order to find the best approach which fits the question, one needs substantial knowledge about them. In the next section, some of these procedures will be introduced. The presented figure shows the main guidelines.

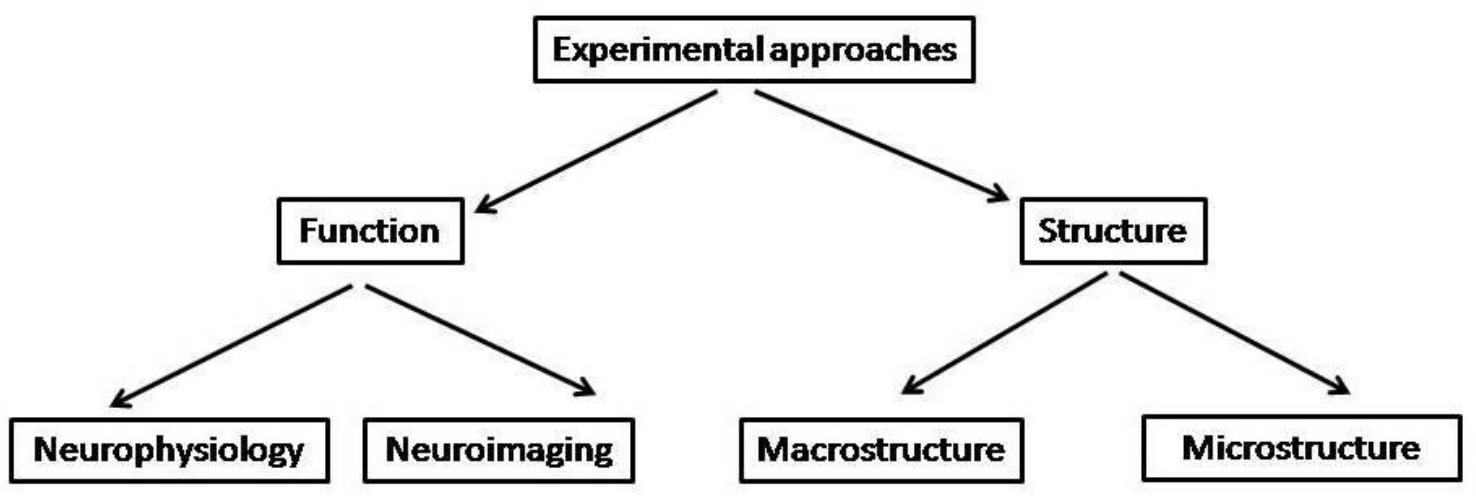

\section{Neurophysiology}

Neurophysiology is one of the first options in neuroscience. Electrophysiological recordings, extracellular single-unit/multi-unit registration (also local field potential studies (LFP)) and more or less psychophysics are included. Centuries of observations led to deductions, e.g., Broca's studies on brain functions and language led him to to discover the lateralization of the brain (Broca, 1861a; Broca, 1861b) or as Berger's invention of the electroencephalograph (EEG)(Berger, 1929), which became a widely used instrument of brain research. 
Psychophysics is the simplest way to study perception. Physical stimuli and the sensation are tested by behavioural performance (Fechner, 1860). In an imaginary experiment, subjects react with a button press if they see a circle on the display. If the experiment is repeated frequently enough, after data processing (for example, use of signal detection theory) personal variability is detectable. By this method, the individual sensitivity of threshold detection or discrimination is defined. Currently, one of the most often used techniques of exploring the brain function is the usage of evoked responses. In EEG, surface electrodes on the head measure the voltage fluctuations of the ionic currents at gyri near the scalp. Averaging several hundreds of time-locked EEG responses generate event-related potentials (ERP) that is a unique tool of neuroscience and also diverse practice in medicine (Luck and Kappenman, 2012). One of the earliest studies of ERP in cognitive neuroscience has presented a new component that was associated to recognition as Contingent Negative Variation (CNV) (Walter et al., 1964) and showed the relevance of EEG waveform (P3) variations about $300 \mathrm{~ms}$ (Sutton et al., 1965) that can be related to thinking.

At the same way, the functional nature of evoked responses from the brain is exhibited by magnetoencephalography (MEG), which records the local current generated magnetic fields that means of superconducting devices. It is used in basic neuroscience (Cohen, 1968) and in neurology (Montez et al., 2009). Both techniques have high temporal resolutions.

Another widely used neurophysiological approach is single-cell/unit recordings. Microelectrodes lowered to the cortex or to the subcortical grey matter close enough to the individual cells or cell-clusters to measure action potentials or local field potentials . This kind of intracerebral electrophysiology provides rich knowledge about cell interactions and the cortical organization (Hubel and Wiesel, 1959; Hubel and Wiesel, 1962; Kuffler, 1953; Wiesel and Hubel, 1963), but has disadvantage as well: negliging the whole brain as a network. In single unit recordings, the activation of a single firing neuron is observed and the spikes are recorded with thin glass or metal (platinum, tungsten), high-impedance microelectrodes (Boulton et al., 1990). Action potential through the neurones causes electric currents at the excitable membrane regions which change the electric charge distribution in and outside of the cells that can be detected with intra- or extracellular electrodes. After proper data processing the separation of units and deeper analysis of the signal is feasible (Thompson and Patterson, 2013). 


\section{Functional neuroimaging}

Similar noninvasive opportunities for gaining information of the working brain are the positron emission tomography (PET) and the functional magnetic resonance imaging (fMRI). During fMRI measurement an indirect signal of neuronal activation is measured. Cell activity is detectable by the changes in the proportion of oxi- and deoxihemoglobin, which cause alteration in the local magnetic property (Ogawa et al., 1990). The relationship between the neuronal activation and blood-oxygen-level dependent (BOLD) signal depends on the neurovascular coupling. Intracerebral electrophysiology in monkeys confirmed that population spikes and local field potentials correlate with the BOLD signal (Logothetis et al., 2001). The evoked fMRI setup is based on different kind of stimulation protocols (visual, auditory, tactile). During the analysis, the voxel-by-voxel measured time-varying BOLD signal and the model predicted response are compared. A significant activation is assumed in those voxels where the signal matches the predicted model (Jenkinson et al., 2012).

Another resource for event related activation is PET which uses radioactive tracers that emit positrons, which interact with an electron annihilating and emitting gamma radiation, which in turn is detected by scintillators (Turkington, 2011). The detection of the gamma photons leaving in opposite direction offers a precise spatial localisation of the tracer. It is widely used in clinical oncology (Beadsmoore et al., 2015) and neuroimaging (Lu and Yuan, 2015).

Summarizing the potentials and weaknesses of the above described methods it is clear that EEG and MEG have an excellent temporal resolution but less effective spatial localization ability. Intracerebral electrophysiology (ICE) owns the best temporal resolution, but the spatial localization is limited by the position of the electrodes. fMRI have much better spatial resolution (in millimetre range), but in most applications the temporal resolution is poor as compared to electrophysiology.

\section{Macrostructure}

In the early era, lesions (Bucy and Kluver, 1955) and brain deformities (Harlow, 1848) provided evidence of functions related to brain areas. Then, direct cortical stimulation studies (Penfield, 1950a; Penfield, 1950b; Penfield, 1957; Penfield, 1958; Rasmussen and Penfield, 1947; Rasmussen and Penfield, 1948) provided evidence of cortical representations. This information is eminent but rough to make subtle deduction of the interactions. Recently, non-invasive neurostimulation protocols are widely used, such as 
transcranial magnetic stimulation (TMS,(Barker et al., 1985), to locally and reversibly interfere with brain functions.

Neuroimaging approaches provide a possibility to deduct the effect of lesions in certain localization in a large population of patients. Voxel-based lesion-symptom mapping (Bates et al., 2003; Kincses et al., 2011) correlates with the clinical features in patients with certain lesion locations. Voxel based comparison of tissue type probabilities can reveal regionally specific atrophy in certain patient populations (Kincses et al., 2014; Stepan-Buksakowska et al., 2014) and is able to link cognitive function to normal variation of brain structure (Draganski et al., 2004a).

\section{Microstructure}

Although, functional imaging experiments offer a unique opportunity for the examination of functional activation in various behavioural conditions, it was recently suggested that behavioural performance is also strongly related to the underlying brain structure. It was shown that the correlation between the individual structural variability and the behavioural performance can identify the involved neuroanatomical structures (Maguire et al., 2000). Moreover, if white matter structure-related parameters are investigated, this approach provides a unique opportunity for the identification of the white matter tracts associated with the certain functions (Floel et al., 2009). Diffusion is the three dimensional Brownian motion of the molecules such as water (Johansen-Berg and Behrens, 2009). In diffusion MRI experiments the diffusion is measured in a short period of time that allows microscopic movements of the water. In spaces bordered by membranes or cell-compartments the diffusion of the water is hindered. Hence, in the white matter the preferred orientation of the diffusion is parallel to the axis of the pathways (Staempfli et al., 2008). Measurement of diffusion in multiple directions allows the characterisation of diffusion in space that can be modelled by a diffusion tensor. Various parameters of this diffusion tensor model can be estimated such as the diffusion along or perpendicular to the main fibre direction, the mean diffusivity or the fractional anisotropy. Such parameters can address the integrity of the white matter microstructure. The relationship between the locally measured diffusion parameters and the behavioural data is capable of revealing the coupling of structure and function (Johansen-Berg, 2010). Human studies have implied that short-term visuo-motor learning enhances the fractional anisotropy (FA) in the cortico-spinal tract (Landi et al., 2011). Steeper learning curves in a sequence-learning task were found to correlate with the local FA (Tomassini et al., 
2011). Recent combined imaging and histological studies demonstrated that plasticity related diffusion parameter changes are related to locally enhanced myelination (Blumenfeld-Katzir et al., 2011; Sampaio-Baptista et al., 2013).

\section{Objectives}

The aim of our studies was to examine the visual system on different levels with alternative methodologies. The following specific questions were addressed:

- How does the neural coding of visual stimuli changes with ageing?

- What is the white matter structural background of the motion detection?

- Could the segregated visual pathways be identified in a double flash illusion experiment? 


\section{Aging alters visual processing of objects and shapes in inferotemporal cortex in monkeys}

Cognitive functions, including visual perception, decline with age. This deterioration of the perceptual processes can be attributed not only to optical defects such as cataract, glaucoma or presbyopia, but also to neurological aging and dysfunction in visual areas. This may have a major impact on the occurrence of home accidents, automobile driving and the quality of life in general (Carter et al., 1997; Desapriya et al., 2014; KallstrandEriksson et al., 2013). Data from psychophysical studies indicate that older people tend to have decreased visual acuity, contrast sensitivity and contour integration (Hutman and Sekuler, 1980; Roudaia et al., 2008; Sekuler et al., 1980). They often demonstrate impairments in visual motion sensitivity, including the perception of apparent motion, and have poorer orientation-judging capabilities (Bennett et al., 2007; Betts et al., 2007; Roudaia et al., 2010)(for a review, see (Andersen, 2012). It has been reported that aging also has an impact on form perception and shape discrimination (Habak et al., 2009; McKendrick et al., 2010; Weymouth and McKendrick, 2012) and figure-background separation (Chee et al., 2006), but it is not clear what changes accompany these impairments.

A number of papers have addressed the connection between aging and the decline of visual functions (Liang et al., 2010; Schmolesky et al., 2000; Spear, 1993; Wang et al., 2006; Wang, 2001; Yang et al., 2008; Yang et al., 2009a; Yu et al., 2006), but we are not aware of any electrophysiological report concerning the effects of aging on the neuronal activity underlying object and shape vision in a high-level visual area such as the monkey inferotemporal cortex (IT).

The macaque monkey has been a useful animal model to study human visual perception. Several papers have compared the visual systems of monkeys and humans (Denys et al., 2004; Orban et al., 2004; Tanaka, 1997; Tompa and Sary, 2010). If we accept that a possible criterion for homologies in different species is the similarity in function and in retinotopy in the particular visual areas, then it is clear that the ventral cortical pathway that serves object vision is conserved across primates into humans (DeYoe et al., 1996; Fize et al., 2003; Kourtzi et al., 2003). A homology for IT in humans is probably the posterior part of the inferotemporal cortex or the lateral occipital complex (for reviews, see: (Orban et al., 2004). 
The aging of monkeys is similar in many respects to human aging. A 30-35-year-old monkey is similar to a human aged 90-95 years (Tigges et al., 1988). On this basis, three human years may be assumed to correspond to one monkey year. Nonhuman primates are ideal for examining central nervous system aging since they share the same neuromorphological pattern as in humans during normal ageing (Gallagher and Rapp, 1997; Lowenstine, 2003). Interestingly, the refractory power of the eye tends to decrease, which is appropriately compensated in monkeys (Fernandes et al., 2003; Ooj and Grosvenor, 1995). This supports the assumption that ocular pathology is not critically relevant in the changes in visual function. We hypothesized that changes in the neuronal coding of objects in IT may partially explain the perceptual deficit of aging monkeys and humans.

\section{Methods}

We compared data obtained from single-cell recordings from monkeys in two age groups: 7 years and 27-33 years (corresponding to 21 and 81-99 years, respectively, in humans). All the participating animals (Macaca mulatta) were engaged in earlier studies in the visual laboratory. The animals performed a simple fixation task and were exposed to the same set of images at a certain stage during their training (for an example of the stimulus set, see (Sary et al., 2006). To compensate for implicit learning, we took our sample from a later phase of the experiments, when the animals could be regarded as experienced or even overtrained. Our behavioural threshold was set to $87 \%$, but at the time of the recording all the animals performed at $>90 \%$ correct level.

Monkeys were examined when they were anaesthetised during the headpost implantation procedure by an ophthalmologist and the same investigation was performed as reported in other papers (e.g., (Yang et al., 2009a)). No major ophthalmoscopic deviations or refractive errors were detected in the lens, vitreous body, vasculature or macula. In addition, monkeys performed at $>90 \%$ correctness in the fixation task that used a few pixel sized fixation point and fixation window of $<1^{\circ}$, which is hardly possible with blurred vision, thus the presence of refractory errors and their corrections were not considered (Fernandes et al., 2003; Ooi and Grosvenor, 1995). It is important to note at this point, that the old animals were purchased and operated on at an old age, thus, it is unlikely that their vision deteriorated rapidly between the surgery and the start of the recordings ( 2 months). 
The four monkeys that participated in the study weighted between 6 and $9 \mathrm{~kg}$ at the time of the experiments. In the young group, the two monkeys were males and the old animals were 2 females. Prior to their training, the animals underwent two session of aseptic surgery. All surgical procedures were carried out under full anesthesia, induced with an i.m. injection of ketamine (Calypsol; $15 \mathrm{mg} / \mathrm{kg}$ ) and atropine $(0.05 \mathrm{mg} / \mathrm{kg}$ ). An endotracheal tube was inserted into the trachea and anesthesia was maintained with Halothane (1-1.2\%), given in a mixture of $\mathrm{N}_{2} \mathrm{O}$ and $\mathrm{O}_{2}$ in a ratio of $2: 1$, or $0.5 \mathrm{mg} / \mathrm{kg}$ i.v. midazolam (Dormicum). An i.v. line was inserted for continuous access and physiological saline was given to compensate for fluid loss. Before the surgical procedure, a preventive dose $(250 \mathrm{mg})$ of the antibiotic ceftriaxone (Rocephin) was given. The incision was infiltrated with local anaesthetic (Procaine). Nalbuphin and nonsteroid anti-inflammatory drugs were administered to the animals postoperatively. The arterial oxygen saturation, expired $\mathrm{CO}_{2}$ level, heart rate and alveolar concentration of the inhalation narcotic were monitored continuously throughout the surgery. A stainless steel headpost was fixed to the head to keep the animal's head stable, and a search coil was implanted under the conjunctiva (Judge et al., 1980) to enable recording of the eye position.

Before the implantation of the registration chamber, all animals underwent T1 and T2 weighted MR acquisitions. Stereotaxic coordinates and individual MR images were used during the surgical procedure to optimise the position of the registration chamber (ant.: 23; lat.: 17). After the surgery, X-ray images were made with the lowered electrode to test the proper location of the electrodes.

During recording, the alternation of white and gray matter during advancing of the electrode, the selectivity for complex colour stimuli, the typical response latency values (Sary et al., 2006; Schmolesky et al., 1998) are a hint for being in the right location. Finally, after the examination, the brains were removed and frozen sections were made to locate the electrode tracts, which pointed to area TE, the lower bank of STS and the lateral part of TE.

The animals performed a fixation task. First, a red fixation point was presented on the monitor (distance: $57 \mathrm{~cm}$ ) for $500 \mathrm{~ms}$, followed by a gray background $(500 \mathrm{~ms})$ and then by the stimulus (500 ms). For each cell, 20 stimuli were used, showing colour reproductions of real world 3D objects or geometric shapes (Sáry et al., 2004). For each of the stimuli at least 10 successful trials (and recordings) were collected in a semirandom fashion. Trials were considered successful if the animal did not break fixation during the trial (fixation window $\sim 0.5^{\circ}$ ). Trials were machine paced; the inter-trial 
interval was set to $1 \mathrm{~s}$. This way, collecting all the required responses lasted for about 7-8 minutes per cell, depending on the number of unfinished trials. Only correct trials were included in the off-line analysis. Once a responsive cell had been isolated, a set of effective and non-effective stimuli were chosen and the neuronal responses were recorded using these stimuli. This allowed us to construct stimulus-preference tuning curves; stimulus preference is a typical characteristic of IT cells. The neuronal activity from the IT was recorded by using standard electrophysiological methods.

Cellular activity was analyzed off-line. All procedures used during surgery and training of the animals conformed fully to the NIH standards and had been approved in advance by the Ethical Council of the University of Szeged.

To characterize the recorded neuronal responses, the following parameters were used:

- Baseline: the mean activity in a time window of $400 \mathrm{~ms}$ preceding stimulus onset.

- The response latency time: the time from stimulus onset to the start of the neuronal activity. We used the Poisson spike train analysis method, which was previously proved to be reliable in different experimental paradigms (Hanes et al., 1995; Sary et al., 2006; Thompson et al., 1996).

- The net firing rate: the mean baseline activity subtracted from the mean overall activity in a 400-ms time window starting $100 \mathrm{~ms}$ after stimulus onset. Baseline activity does not change immediately after stimulus presentation, thus, if net responses are considered, like in this study, a certain time after stimulus onset might be omitted. The mean response latency time in IT being $100 \mathrm{~ms}$, it is a general convention to leave out the first $100 \mathrm{~ms}$ or so starting at stimulus onset (e.g., (Missal et al., 1999; Op de Beeck et al., 2001; Sary et al., 1993; Tamura and Tanaka, 2001). This procedure does not have influence on the results, in contrary, removes some noise from the data.

- The evoked-to-spontaneous ratio: a value calculated by dividing the net firing rate by the baseline activity. This shows how much the stimulus-related activity differs from the background activity.

- Fano factor: the standard deviation of probability distribution of the spike counts. This is one of the most widely used statistical parameters to describe the variability of the spike trains (Churchland et al., 2010). 
The following factors are considered special to neurons which play roles in the sensory processing and discrimination of, for example, the speed and direction of movement or shapes.

- The selectivity index (SI): this is used to describe the stimulus preference of cells. $\mathrm{SI}=\left(R_{\max }-R_{\min }\right) /\left(R_{\max }+R_{\min }\right)$

where $R_{\max }$ is the maximal and $R_{\min }$ is the minimum response, respectively, to a particular stimulus set. The closer the value is to 1 , the better the cell discriminates between a preferred and a non-preferred stimulus.

- The depth of selectivity index (DSI). Long-term recordings have indicated that the cellular responses can best be characterized by a variable derived from the median of the firing rate (Bondar et al., 2009). In the optimal case, i.e. if the cell shows a stimulus preference, the value is 1 or close to it. It is a normalized variable with the greatest response taken as 1 and the median of the responses being subtracted from it:

$$
\mathrm{DSI}=\left[n-\left(\sum_{i=1, n} R_{i} / R_{\max }\right)\right] /[n-1],
$$

where $R_{i}$ is the $i$ th response to the $n$ stimuli, while $R_{\max }$ is the maximum response. The value ranges from 0 to 1 , where the highest value indicates that the examined cell reacts to only one stimulus.

- The sparseness index (SP): the ratio between the used and effective stimuli, i.e. the "tail" of the distribution of the cellular responses to the stimulus set (Rolls and Tovee, 1995).

$\mathrm{SP}=\left[\Sigma_{i=1, n}\left(R_{i} / n\right)\right]^{2} /\left[\sum_{i=1, n}\left(R_{i}^{2} / n\right)\right]$,

where $R_{i}$ is the $i$ th response from the responses to a stimulus set containing $n$ stimuli. The value can range between 0 and 1 . A small SP value means that there are only a few stimuli in the set which can evoke large responses (i.e. there is a stimulus preference), while a large SP values means that most of the stimuli can trigger cellular responses which do not differ too much.

\section{Statistics}

Nonparametric tests were used throughout the analysis. To verify the differences between groups, we performed a Mann-Whitney U test. Results (U) were compared with the corresponding value of the $\chi^{2}$-table at the same degree of freedom (df). Results were taken as significant if the type I error was less than $5 \%(p<0.05)$. All values are presented with the median and the interquartile range in the text. Correction for multiple 
comparisons was applied according to the Bonferroni method. In some cases, however, we present both corrected $\left(p_{\text {corr }}\right)$ and uncorrected values, as we consider that the Bonferroni correction is too conservative and could mask relevant results. For this reason a nonparametric permutation test was implemented (number of permutations $=5000$, (Winkler et al., 2014). Cluster analysis (Ward method) was used to reveal the relationship between possible groups among the variables.

\section{Results}

We recorded a total of 288 neurons from 4 Macaca mulatta monkeys to investigate the functional consequences of neuronal aging. Two groups of data were formed for comparison: a young group ("Young", 2 monkeys, aged 7 years, number of cells: 221) and an old group (“Old”, 2 monkeys aged 27 and 33 years, respectively, number of cells: $67)$.

First to have an initial analysis of our dataset we ran a cluster analysis to determine whether our data form groups according to the age or not. Baseline activity, as the raw data with no modulation was chosen to explore the internal pattern of the groups. Second, latency data were also used, as our hypothesis was that this is a primary measure of cell aging (Kuba et al., 2012; Wang et al., 2005). The cluster analysis revealed two main clusters, one comprising the data on the 2 Old animals and the other the data on the Young ones.

In the next step, we compared the data on all the registered cells by means of the MannWhitney $U$ test. The baseline of firing rate did not significantly differ in the 2 groups: $6.00(2.81-9.69)$ spikes/s in the Young, and $5.23(2.50-10.27)$ spikes/s in the Old animals, respectively.

The evoked-to-spontaneous ratio was 3.63 (2.19 - 6.10) in the Young and 3.26 (2.34 6.75 ) in the Old group. Once again, there was no significant difference between the groups.

The Fano-factor did not indicate differences between the groups either: $1.26(0.76-1.85)$ and $1.31(0.84-2.00)$ for the Young and Old groups, respectively.

The net firing rates were likewise not different: 15.67 (8.33 - 25.33) spikes/s vs. 14.33 (8.33 - 24.66) spikes/s, respectively, in the Young-Old comparison.

On the other hand, the latency revealed a difference between the Young and Old groups (Table 1): 128.73 (96.88 - 161.86) ms and 147.20 (124.54 - 179.83) ms, respectively; $\mathrm{U}=$ 4101.00; $p_{\text {corr }}<0.05$. 
SI too demonstrated a difference $\left(\mathrm{U}=4810.50 ; p_{\text {corr }}<0.001\right)$ in the Young vs. Old comparison : $0.89(0.69-0.97)$ and $0.76(0.47-0.90)$, respectively.

The SP values for the Young-Old groups (Table 1) were $(0.47$ (0.28 - 0.73) and 0.75 (0.56 - 0.90), respectively), this difference was significant, according to the Mann-Whitney test $\left(\mathrm{U}=4245.50 ; p_{\text {corr }}<0.001\right)$.

DSI similarly pointed to a group difference in the Young-Old comparison: (0.60 (0.44 $0.79)$ vs. $0.46(0.31-0.68)$, respectively; $\left.\mathrm{U}=5197.00 ; p_{\text {corr }}<0.01\right)$.
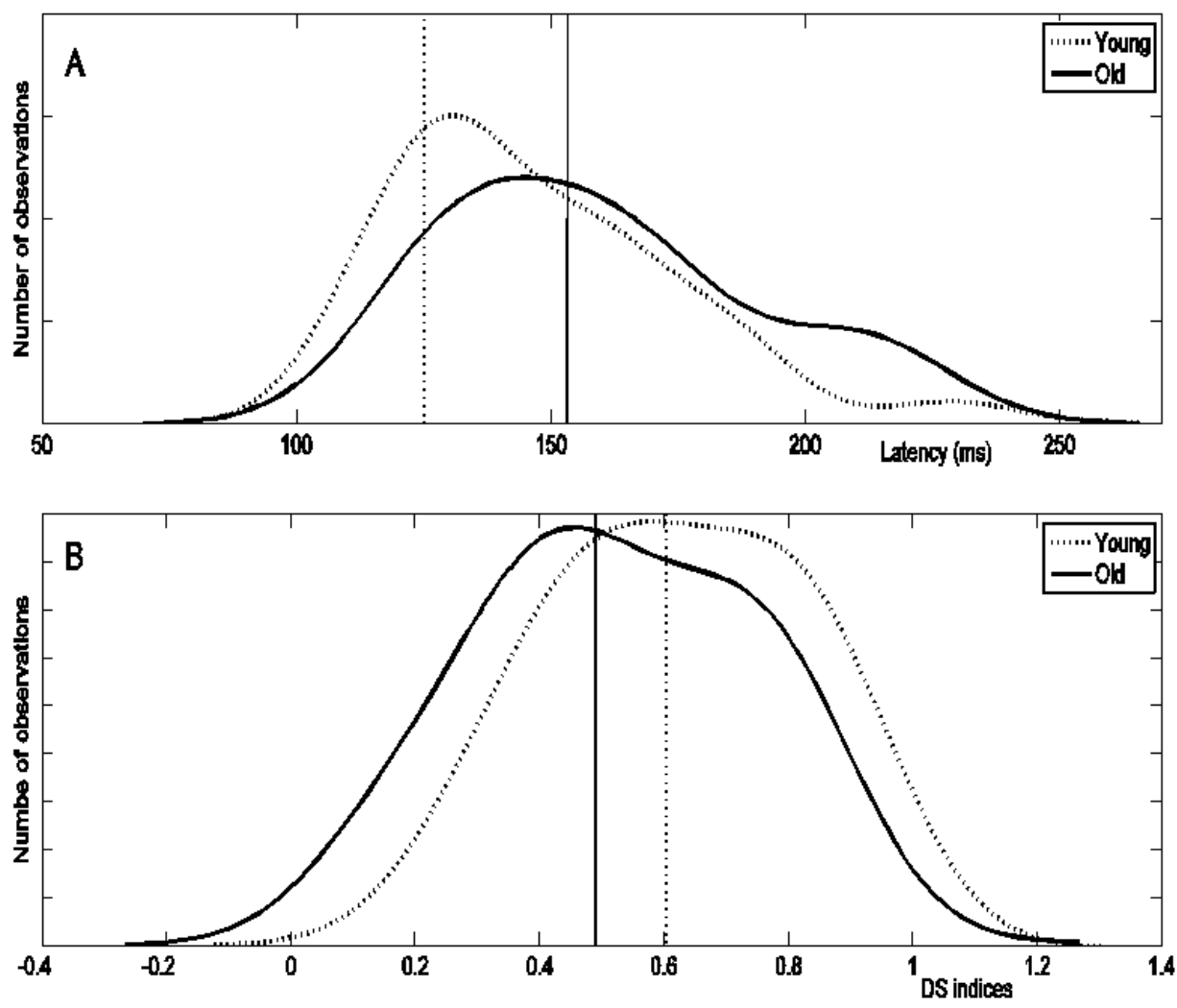

1. Fig. Graphs showing the distribution of latency data (panel A) and the DSI values (panel B). Dotted lines on both graphs show data and median values of the Young, continuous line the data and median values of the Old group, respectively.

To reduce the biasing effects of unequal samples, we repeated the analysis on 22 randomly chosen cells from each animal, i.e., 44 cells from each age group. Cells were selected from that stage of the experiments when the animals were considered overtrained. During this phase, the animals worked on a daily basis, achieving a $>90 \%$ performance rate in the task, and the effect of attentional fluctuation could therefore be minimized.

As in the analysis involving all the cells, the response latency values of the Young and Old data were statistically different: 124.85 (97.00 - 161.74) ms and 154.51 (129.33 - 
178.64) $\mathrm{ms}$, respectively $\left(\mathrm{U}=567.00 ; p_{\text {corr }}<0.01\right)$. Also, the $\mathrm{SI}$ values pointed to a significant difference between the groups $(\mathrm{U}=713.50 ; p<0.034)$ before the Bonferroni correction, which disappeared after it $\left(p_{c o r r}<0.27\right)$. Since in our opinion, this difference is essential for understanding neuronal aging and the decrease in perceptual performance, we rerun this comparison with the Monte - Carlo method in addition to the Bonferroni correction ( $\mathrm{p}_{\text {permutation }}<0.027$ ). The neuronal selectivity values in the two groups were: $0.90(0.69-0.97)$ vs. $0.81(0.51-0.92)$. As for the DSI values, the same procedure masked the result $\left(U=670.00 ; p<0.013, p_{\text {corr }}<0.10\right)$. However, after randomization, the differences were significant $(<0.02)$.

The SP parameters, were significantly different $\left(\mathrm{U}=587.00 ; p_{\text {corr }}<0.05\right): 0.46(0.29$ $0.73)$ vs. 0.75 (0.51 - 0.88), in the Young and Old groups, respectively. 


\section{An investigation of the white matter microstructure in motion detection using diffusion MRI}

The neural substrates of motion sensitivity are located at different levels of the visual system. Electrophysiological investigations have revealed that in the retina subgroups of ganglion cells react differently to moving lights, some responding with activation and others with decreased firing (Sivyer et al., 2010). Similarly, the lateral geniculate nucleus (LGN) (Stanley et al., 2012), the striate cortex (Beckett et al., 2012) and some peri- and parastriate areas (Larsson et al., 2010; Sary et al., 1995) respond to a dot moving across the receptive fields of the neurons. Higher stages are presented in the area of V5/hMT+ (Boussaoud et al., 1990; Morrone et al., 2000), which participates in motion and depth perception, eye-movements, detection of speed, computing pattern motion, integrating direction of motion (Born and Bradley, 2005) in spatial properties processing (Lui and Rosa, 2015) and extracting structure from motion (Siegel and Andersen, 1988).

Motion transparency (e.g. two motions superimposed on each other) applied in the form of a random-dot kinematogram is frequently used to identify motion-sensitive cortical regions in functional imaging (Friston et al., 1997) and electrophysiological experiments (Antal et al., 2005; Braunitzer et al., 2012). It offers an opportunity for the analysis of integrated action vision in close-to-real situations. Moreover, through modulation of the attention to the various components of the task the mechanism, by which attentional networks interacts with motion detection areas can be revealed (Buchel et al., 1998; Kellermann et al., 2012).

Most of the available information on theV5/MT+ originates from monkey experiments, human fMRI and positron emission tomography (PET) studies. Such knowledge is mostly functional in nature, but the structural background of motion detection is not sufficiently well known, especially that regarding the white matter pathways.

\section{Methods}

\section{Subjects}

Sixteen healthy volunteer subjects (average age was 26.5 (range: 21-40 years); 9 female, 11 right-handed, mean body mass index $=22.05 \pm 4.22$ ) participated in the study. None of 
them suffered from any neurological or psychiatric diseases. All subjects had normal or corrected-to-normal (5/5) visual acuity.

\section{Ethics}

All study participants provided their written informed consent in accordance with the Declaration of Helsinki; the study was approved by the ethics committee at the University of Szeged (Ref. no.: 87/2009).

\section{Psychophysical test}

The motion detection threshold was measured with a random-dot kinematogram. Stimuli were generated with Psychophysics Toolbox Version 3 (http://psychtoolbox.org/), under Matlab (The MathWorks, Inc.) on a PC, and presented on a 24-inch LCD monitor at a resolution of 1920 by 1200 pixels and at a $60 \mathrm{~Hz}$ refresh rate. The stimuli were 100 moving black dots in random positions with variable coherence rates. Stimuli were presented on a neutral grey background in a rectangular stimulation field occupying $60 \%$ of the whole screen. Subjects were seated at $0.5 \mathrm{~m}$ from the screen and the stimulation field subtended an area of 35.74 by 22.34 visual angles. The diameter of each dot was 10 pixels $(\sim 3 \mathrm{~mm})$. In each trial, a given percentage of the dots moved coherently to the right or to the left, while the remainder moved in random directions. After each trial, movement-starting points were generated de novo. One trial lasted approximately for $0.8 \mathrm{~s}$ (50 consecutive frames), during which each dot travelled a distance of $38.4 \mathrm{~mm}$. (For more details see: (Braunitzer et al., 2012)). The task of the subjects was to indicate whether the coherently moving dots moved to the left or to the right by pressing the appropriate cursor button on the keyboard. The absolute coherence threshold was determined by the QUEST adaptive threshold-seeking algorithm (Watson and Pelli, 1983).

\section{Data acquisition:}

Neuroimaging data acquisitions were carried out on a 1.5 T GE Signa Excite HDxt MR Scanner (GE Healthcare, Chalfont St. Giles, UK). Three-dimensional spoiled gradient echo images (FSPGR: echo time [TE]: $4.1 \mathrm{~ms}$; repetition time [TR]: $10.276 \mathrm{~ms}$; matrix: $256 * 256$; field of view [FOV]: $25 \mathrm{~cm} * 25 \mathrm{~cm}$; flip angle: 15 degrees; in-plane resolution: $1 \mathrm{~mm} * 1 \mathrm{~mm}$; slice thickness: $1 \mathrm{~mm}$ ) and 60-direction diffusion-weighted images with 6 non-diffusion-weighted reference volumes (TE: $93.8 \mathrm{~ms}$; TR: $16.000 \mathrm{~ms}$; matrix: $96 * 96$; FOV: $23 \mathrm{~cm} * 23 \mathrm{~cm}$; flip angle: 90 degrees; in-plane resolution: $2.4 \mathrm{~mm}$ 
* $2.4 \mathrm{~mm}$; slice thickness: $2.4 \mathrm{~mm}$; b: $1000 \mathrm{~s} / \mathrm{mm}^{2}$; number of excitations [NEX]: 2; array spatial sensitivity encoding technique [ASSET]) were acquired for all subjects.

\section{Data analysis:}

\section{Correlation of diffusion parameters with behavioural measures}

Diffusion data were corrected for eddy currents and movement artefacts by 12 degree of freedom affine linear registration to the first non-diffusion-weighted reference image. An algorithm included in the FMRIB Diffusion Toolbox (FDT) of FSL (v.4.0) fitted diffusion tensors at each voxel (Smith et al., 2004). The FA was computed for the whole brain. In order to reduce the possible errors arising from misalignment of the images, we used the Tract Based Spatial Statistics (TBSS) method (Smith et al., 2007). The FA images for all subjects were aligned into a common space with the non-linear registration tool FNIRT, which uses a b-spline representation of the registration warp field. A mean FA image was created and the threshold was set at $\mathrm{FA}=0.2$, yielding a mean FA skeleton at the centres of all tracts common to the group. The aligned FA data for each subject were then projected onto this skeleton and the resulting data were fed into voxel-wise cross-subject statistics. Modelling and inference by using the standard general linear model (GLM) design set-up was accomplished by using permutation-based cluster analysis $(\mathrm{n}=5000)$ as implemented in the FSL software package (Nichols and Holmes, 2002). The design was encoded for the motion detection threshold value. Statistical thresholding was carried out with a novel method of Threshold Free Cluster Enhancing (Smith and Nichols, 2009). Since a correlation was expected only in well-circumscribed regions of the visual and attention networks, it would have been over-conservative to correct for multiple correlations based on the total number of voxels in the skeleton. Hence, we used the non-corrected statistics thresholded at the $1 \%$ significance level. Only clusters larger than 4 voxels were considered for further analysis and discussion.

\section{Structural connectivity}

Connectivity of the regions showing a significant correlation with the motion detection threshold was defined by probabilistic tractography (FDT, part of FSL: www.fmrib.ox.ac.uk/fsl/fdt). A multifibre diffusion model was fitted that estimated the probability distribution in the direction of 1 or more fibre populations at each voxel (Behrens et al., 2007). Probabilistic tractography was then performed from any brain voxel by tracing streamline samples through these probabilistic distributions in the fibre 
direction. For tractography, we generated 5000 streamline samples from each seed voxel to build up a connectivity distribution. The number of these samples passing through each brain voxel was interpreted as proportional to the probability of connection to the seed voxel. By fitting a multifibre model to our diffusion data, we were able to trace pathways through regions of fibre crossing (Behrens et al., 2007). The seed masks were the binary masks of the suprathreshold clusters of the TBSS analysis. The result of the tractography was registered to standard space, binarised and summed over subjects for visualisation.

\section{Results}

\section{Correlation of the white matter integrity and the motion detection threshold}

The mean motion detection threshold was $18.87 \pm 5.79 \%$ coherent motions. A significant positive correlation was found between the motion detection threshold and the local FA in the right frontal cortex in the posterior part of the right superior frontal gyrus $(p<0.0032$, non-corrected), the right juxta-cortical superior parietal lobule ( $\mathrm{p}<0.0032$, non-corrected), the left parietal white matter $(\mathrm{p}<0.001$, non-corrected), the left superior temporal gyrus ( $\mathrm{p}<0.0026$, non-corrected) and the left optic radiation ( $\mathrm{p}<0.0036$, non-corrected).

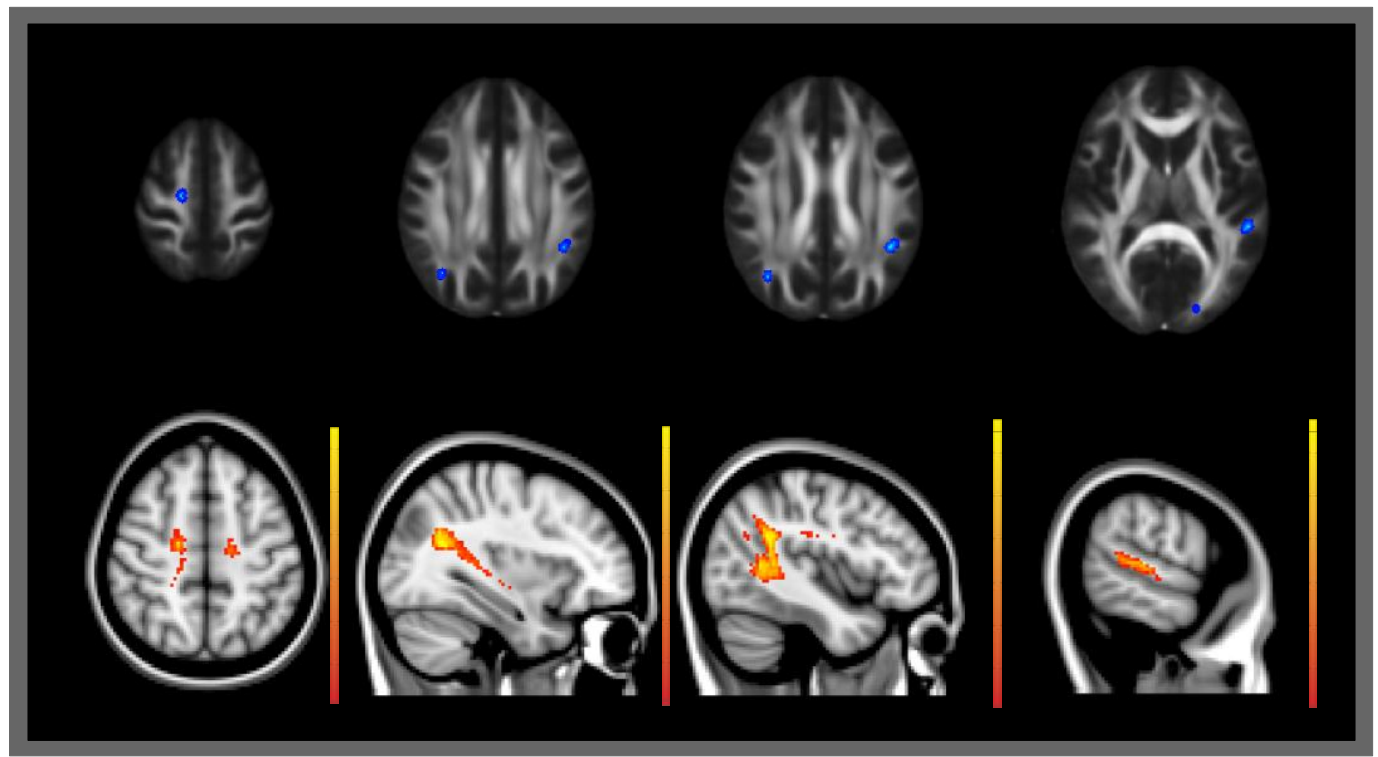

2. Fig. The upper row shows regions of significant positive correlation between voxel-wise fractional anisotropy and the motion detection threshold $(\mathrm{p}<0.01$, uncorrected; clusters are thickened for better visualisation). Statistical images are overlaid on the FMRIB58 standard brain. The lower row indicates the connectivity of the regions in the upper row. Labels (rSFG: right superior frontal gyrus; rSPL: right superior parietal lobe; IPOTj: left parieto-occipito-temporal junction; ISTG: left superior temporal gyrus) indicate the seed regions of the tractographies depicted in the lower row. Individual probabilistic tractography is thresholded at 500 particles, binarised and summed across subjects in MNI space. Various thresholds are chosen for each tractography results for better visualisation. 
Connectivity of the white matter region having correlated microstructure with behavioural data

The right frontal cluster showed strong connections to the identical region in the left hemisphere and to the right cortico-spinal tract, and a smaller pathway leading to the right parietal lobe. The right superior parietal cluster exhibited the strongest connectivity to the right superior temporal gyrus. The cluster in the left parietal white matter had strong connections to the angular gyrus, the frontal white matter (through the putative superior longitudinal fascicle) and the occipito-temporal junction. The cluster in the superior temporal gyrus had connections to the angular gyrus, which led further to the frontal cortex through the arcuate fascicle, and a smaller fibre tract connected the cluster to the parietal white matter under the precuneus.

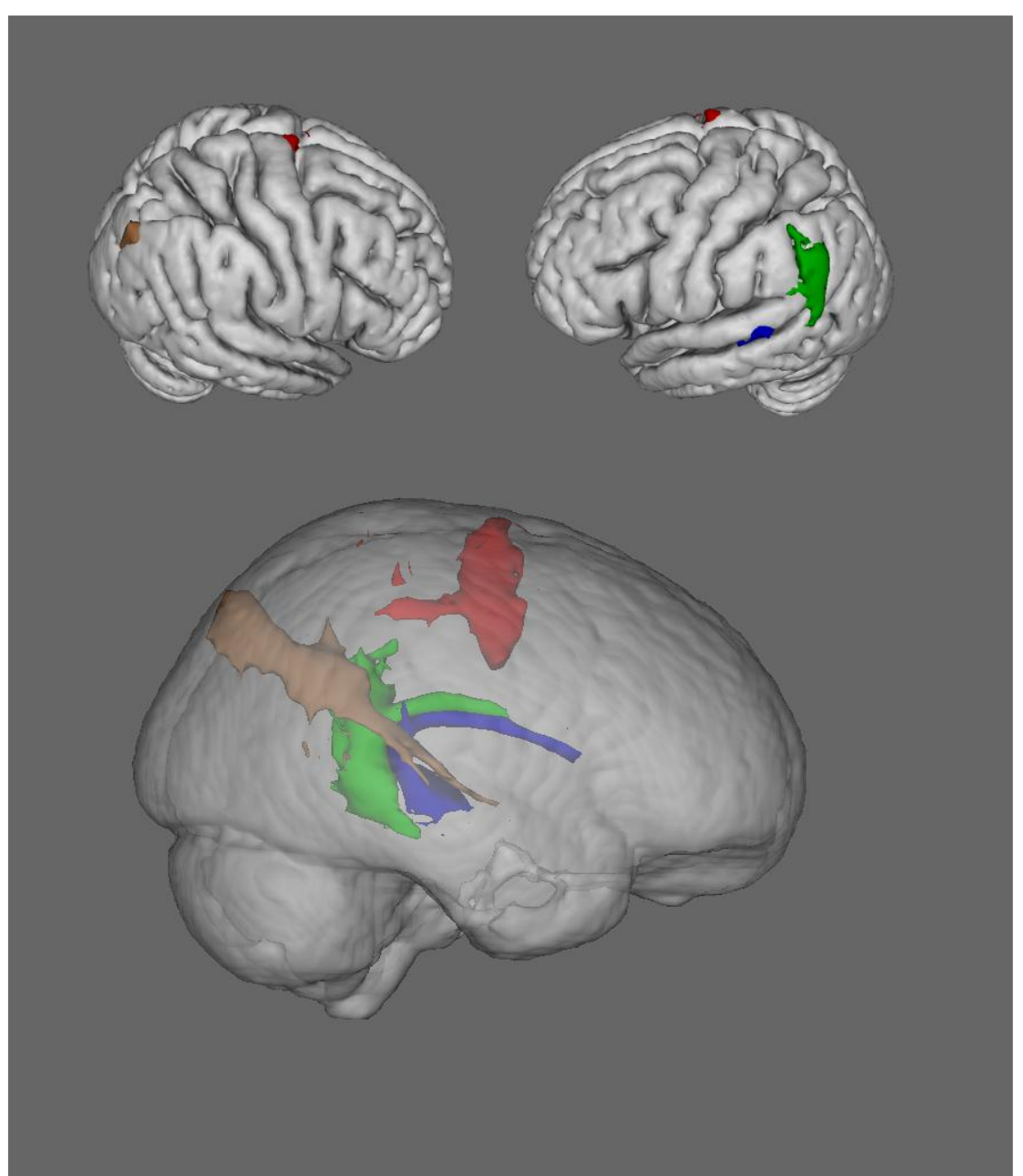

3. Fig. The lower image indicates the pathways; the upper two images (right and left views) show the cortical projections. The inferior and medial branches of the superior longitudinal fascicle on the left are indicated in blue and green. The superior branch of the right superior longitudinal fascicle and part of the cortico-spinal tract originating from the putative frontal-eye field are indicated in red. The connection on the right, between the superior parietal lobule and the superior temporal gyrus, is indicated in brown. 


\section{Audio-visual integration through the parallel visual pathways}

One brief flash and two short tones presented simultaneously induce the illusion of a second flash, called the double flash illusion. Since the first description of the phenomenon (Wilson, 1987), there have been several studies of the possible background mechanisms. Event-related potential studies demonstrated that auditory stimuli could influence the activity of the visual cortex (Shams et al., 2001; Teder-Salejarvi et al., 2002) and an illusory flash-related gamma burst was found in primary visual areas (Bhattacharya et al., 2002; Mishra et al., 2007). Functional MRI investigations revealed illusory flash-related brain activity in the superior colliculus, the primary visual cortex, and the right superior temporal sulcus (STS) (Watkins et al., 2006). These findings suggest that such interaction of the bimodal information could be based on the communication between the primary visual cortex, the STS and the primary auditory cortex (Mishra et al., 2007; Watkins et al., 2006; Watkins et al., 2007). Earlier studies suggested that the double flash illusion is primarily mediated by the dorsal pathway. However, it has not yet been investigated whether the phenomenon can be evoked over both visual streams, and no systematic investigation of the involved neural structures of the dorsal and ventral streams in the illusion has yet been performed. The segregated visual pathways can easily be distinguished by modulating the contrast of the stimuli: stimuli with high contrast and colour are processed predominantly in the ventral stream, while stimuli with low contrast are processed in the dorsal stream (Brannan and BodisWollner, 1991; Legge, 1978).

In this study we investigated the contributions of the parallel visual pathways to the double flash illusion, and aimed to identify the structural background of this processing. Using auditory stimuli to trigger the phenomenon, we set out to induce the illusion with (i) low-contrast stimuli to drive the dorsal pathway, which is sensitive to contrast and insensitive to colour information and, (ii) isoluminant stimuli to drive the ventral pathway, which is less sensitive to contrast differences. Although the distinction is not complete, this approach might be useful to investigate how additional auditory information can influence the different parts of the visual system (Kaposvari et al., 2014). 


\section{Methods}

\section{Subjects}

Sixteen healthy subjects (10 females; mean age of 27.4 years) were enrolled in the study. None of them suffered from any neurological or psychiatric diseases. All of them had normal or corrected-to-normal (5/5) visual acuity and good colour vision. The study was approved by the ethical committee of the University of Szeged (Ref. no.: 87/2009) and all study participants gave their written informed consent in accordance with the Declaration of Helsinki.

\section{Behavioural test}

The subjects sat in a sound-attenuated dark room with their heads resting on a chin and forehead support. Their eyes were $57 \mathrm{~cm}$ away from the screen (ViewSonic monitor, 21" diameter, $800 \times 600,60.0 \mathrm{~Hz}$ ) and the speakers. The two computer speakers were positioned symmetrically, on each side of the monitor, at $25^{\circ}$ from the fixation point. The subjects had to fix their gaze on a black fixation dot at the middle of the monitor. A disc subtending a visual angle of $1.5^{\circ}$ was displayed in a peripheral position at 9.25 eccentricity below the fixation point, as a visual stimulus (Watkins et al., 2007).

Two conditions were investigated: an isoluminant (IL) condition, where a red disc was presented on a green background $\left(8.9 \mathrm{~cd} / \mathrm{m}^{2}\right)$, and a low-contrast (LC) condition, where we used a light-grey disc $\left(9.7 \mathrm{~cd} / \mathrm{m}^{2}\right.$, contrast $\left.9 \%\right)$ on a darker grey background $(8.9$ $\left.\mathrm{cd} / \mathrm{m}^{2}\right)$. The conditions were presented in a semi-random sequence.

The conditions contained 6 subconditions: 6 variations of flashes (one flash, one flash with one tone, one flash with two tones, two flashes, two flashes with one tone, and two flashes with two tones). One subcondition consisted of 40 repetitions of the trial, and thus one block contained 240 semi-random-presented trials.

The presentation of the trial started with the condition-specific green background in the IL condition and the grey background in the LC condition. After $200 \mathrm{~ms}$ one or two discs were presented for $17 \mathrm{~ms}$ on this background, without or with one or two tones, according to the given condition. The stimulus onset asynchrony (SOA) between two flashes was 85 ms. The duration of the tones $(3.5 \mathrm{kHz}, 70 \mathrm{dBSPL})$ was $10 \mathrm{~ms}$ and was presented at the same time as the first flash. The SOA between the two tones was $85 \mathrm{~ms}$. The previously mentioned experiments used auditory and visual stimuli slightly shifted in time, but as reported, the two designs with simultaneously presented or shifted stimuli resulted in only slight differences (Watkins et al., 2006). After the presentations of the flashes and tones, 
the subject had to decide whether one or two discs were displayed, independently of the tones, and press the left (one flash) or right arrow (two flashes) button on the keyboard with the dominant hand. After the subject had pressed a key, the grey background (8.9 $\mathrm{cd} / \mathrm{m}^{2}$ ) appeared for the inter-trial interval of $1000 \mathrm{~ms}$. Feedback concerning the correctness or not of the responses was not provided.

\section{Analysis of behavioural data}

Signal detection theory was used to analyse the behavioural results. With this method, the sensitivity of the subjects towards the visual stimuli could be described. The aim was to verify that the illusions were caused by changes in perceptual sensitivity, but not by the general response bias. The sensitivity is expressed as

$d^{\prime}=z(H)-z(F)$

where $d^{\prime}$ is the sensitivity, and $z$ is the inverse of the normal cumulative distribution. In one block, we calculated three $d^{\prime}$ values for two subconditions. The correct identification of the second flash was accepted as a 'hit' $(\mathrm{H})$; when the subject reported one flash instead of two, it was recorded as a 'miss'. When one flash was reported as two, we accepted it as a 'false alarm' $(F)$ and the correct identification of one flash was accepted as a 'correct rejection'. To calculate the $d$ ' value for the control, we used the two subconditions without tones (one flash and two flashes). For double flashes, we used the two subconditions with two tones (one flash with two tones and two flashes with two tones). To examine the power of the illusions, we compared the control $d^{\prime}$ value with that for fusion or the double flash by the paired t-test (Watkins et al., 2006). A criterion $(C)$ was calculated to indicate the response bias via the expression (MacMillan and Creelman, 2005):

$C=-[z(p H)+z(p F)] / 2$,

A positive value of $C$ indicates the bias when the subjects rather report one flash, while a negative value indicates when they rather report two flashes.

\section{Data acquisition}

Neuroimaging data acquisitions were carried out on a 1.5 T GE Signa Excite HDxt MR Scanner (GE Healthcare, Chalfont St. Giles, UK). Three-dimensional spoiled gradient echo images (FSPGR: echo time [TE]: $4.1 \mathrm{~ms}$; repetition time [TR]: $10.276 \mathrm{~ms}$; matrix: $256 * 256$; field of view [FOV]: $25 \mathrm{~cm} * 25 \mathrm{~cm}$; flip angel: 15 degree; in-plane resolution: 
1*1 $1 \mathrm{~mm}^{2}$; slice thickness: $1 \mathrm{~mm}$ ) and 60 directions diffusion-weighted images with 6 nondiffusion-weighted reference volume (TE: $93.8 \mathrm{~ms}$; TR: 16,000 ms; matrix: 96*96; FOV: $23 * 23 \mathrm{~cm}^{2}$; flip angle: 90 degree; in-plane resolution: $2.4 * 2.4 \mathrm{~mm}^{2}$; slice thickness: 2.4 $\mathrm{mm} ; \mathrm{b}: 1000 \mathrm{~s} / \mathrm{m} 2$; number of excitations [NEX]: 2; array spatial sensitivity encoding technique [ASSET]) were acquired for all subjects.

\section{Data analysis}

Diffusion data were corrected for eddy currents and movements artefacts by twelve degrees of freedom affine linear registration to the first non-diffusion-weighted reference image. Diffusion tensors at each voxel were fitted by an algorithm included in FMRIB's Diffusion Toolbox (FDT) of FSL (v.4.0) (Smith et al., 2004). Fractional anisotropy (FA) was computed for the whole brain. In order to reduce the possible errors arising from misalignment of the images, we used the Tract Based Spatial Statistics (TBSS) method (Smith et al., 2007). For all subjects, the FA images were aligned into a common space, using the non-linear registration tool, FNIRT, which uses a b-spline representation of the registration warp field. The mean FA image was created and then thresholded at FA=0.2, deriving a mean FA skeleton representing the centres of all tracts common to the group. The aligned FA data for each subjects were then projected onto this skeleton and the resulting data were fed into voxel-wise cross-subject statistics. Modelling and inference with the standard general linear model design set-up was accomplished by using permutation-based cluster analysis $(n=5000)$ as implemented in the FSL software package (Nichols and Holmes, 2002). The design encoded for the differences of the $d^{\prime}$ value of the double-flash and the control $d^{\prime}$ in two conditions (isoluminant and low-contrast) from psychophysical measurements. Statistical images were thresholded by threshold free cluster enhancement (Smith and Nichols, 2009). Since our task was a relatively low level perceptual task, it would have been over-conservative to correct for multiple correlations based on the total number of voxels. Hence, we used the non-corrected stats thresholded at a $1 \%$ significance level. Only clusters larger than 5 voxels were considered for discussion.

The connectivity of the regions was significantly correlated with the behavioural data, defined by the probabilistic tractography (FDT, part of FSL: www.fmrib.ox.ac.uk/fsl/fdt). A multifibre diffusion model was fitted that estimated the probability distribution in the direction of 1 or more fibre populations at each voxel (Behrens et al., 2007). Probabilistic tractography was then performed on any brain voxel by tracing streamline samples 
through these probabilistic distributions in the fibre direction. For tractography, we generated 5000 streamline samples from each seed voxel to build up a connectivity distribution. The number of these samples passing through each brain voxel is interpreted as proportional to the probability of the connection to the seed voxel. By fitting a multifibre model to our diffusion data, we were able to trace pathways through regions of fibre crossing (Behrens et al., 2007). The seed masks were binary cluster-masks of the TBSS analysis.

\section{Results}

\section{Behavioural data}

The paired t-test between d' values, given by using Signal Detection Theory, in control and double-flash conditions indicated significant results in both the low contrast (LC) condition ( $\mathrm{p}<0.0003)$ and the isoluminant (IL) condition $(\mathrm{p}<0.0027)$.

The criterion revealed significant $(\mathrm{p}<0.01)$ negative bias for the double flash as compared with the control criterion in both conditions (Table 1). This shows that two tones biased the participants to report two flashes instead of one for double-flash illusions, but not the general response bias.

\section{Correlation of behavioural data with white matter microstructural integration}

In the IL condition, a significant positive correlation of the behavioural data (the likelihood of perceiving a double-flash illusion) and the fractional anisotropy (FA) was found in the juxtacortical infero-temporal white matter on the right and in the bilateral insulae by the Tract-Based Spatial Statistic (TBSS) analysis.

In the LC condition, a significant positive correlation was observed between FA and the likelihood of perceiving a double-flash illusion in the juxtacortical white matter of the parieto-occipital junction on the right side, the right frontal white matter under the superior frontal gyrus and bilaterally in the deep cerebellar white matter. 


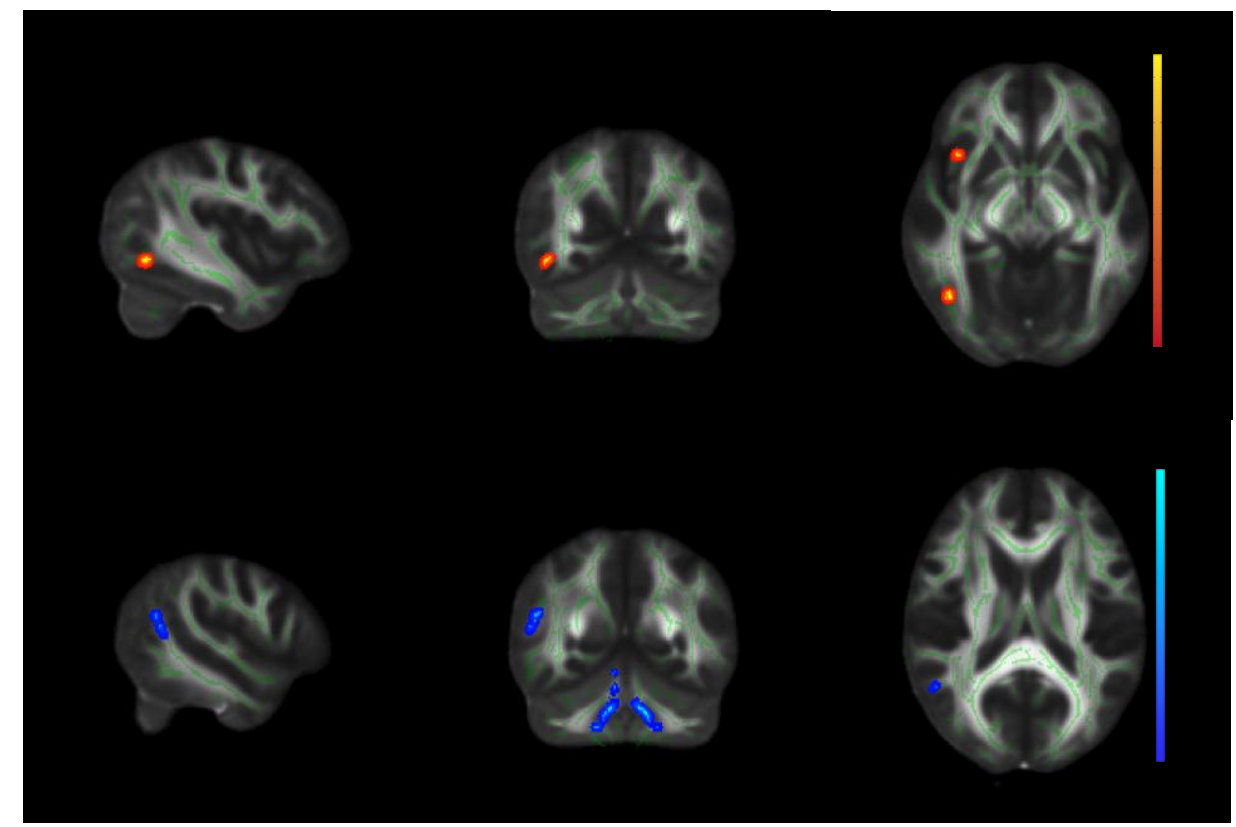

4. Fig. Correlation of FA with a higher likelihood of perceiving a double-flash illusion. White matter microstructure as measured by FA, showed correlation with the perceptual sensitivity to double flash illusion in the isoluminant (upper row) and low-contrast condition (lower row). The identified white matter regions overlap with the ventral and dorsal visual pathways respectively. Images are overlaid on the FMRIB58_FA standard image. The mean FA skeleton, thresholded at 0.2, is depicted in green shades. Significant clusters a re indicated in red for the isoluminant and in blue for the low-contrast condition.

\section{Connectivity analysis}

The probabilistic tractography from the infero-temporal white matter region revealed a high correlation with the likelihood of perceiving a double-flash illusion in the IL condition, showed tracks running along the inferior border of the temporal lobe through the inferior fronto-occipital fascicle and the inferior longitudinal fascicle.

In the LC condition, the tractography initiated from the juxtacortical parieto-occipital cluster of the TBSS analysis showed fibres along the putative arcuate fascicle, running towards the frontal lobe. 


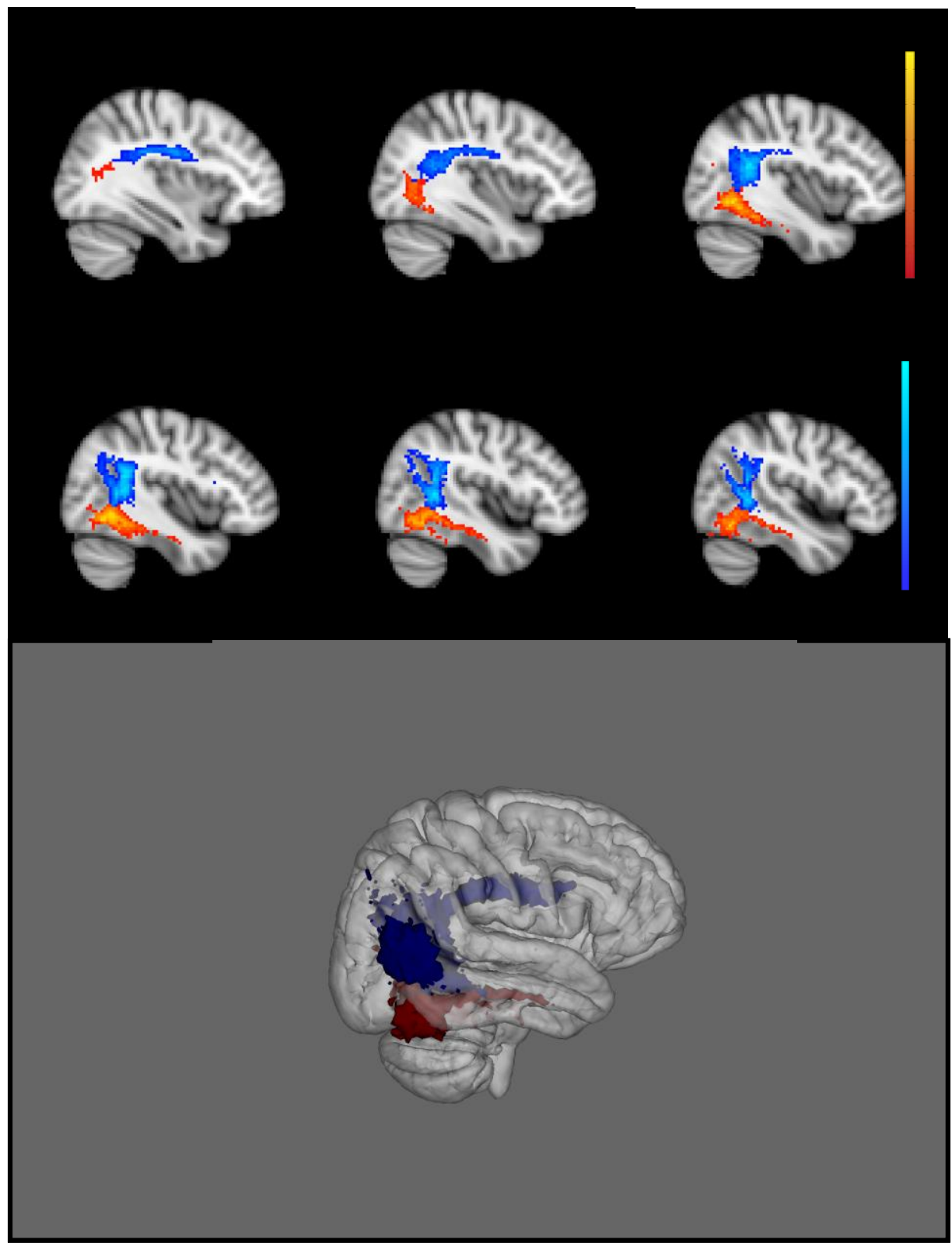

5. Fig. Connectivity of clusters showing the correlation between FA and behavioural data in the isoluminant (red) and low-contrast (blue) conditions. The white matter fibres identified by the tractography correspond to the ventral and dorsal visual pathways in the isoluminant and low-contrast conditions respectively. The binary cluster masks were used as seed mask for each subject. Five thousand streamline samples from each seed voxel were drawn to build up a connectivity distribution that was thresholded for 1000 particles for each subject and binarized. Population connectivity maps were derived for controls by adding these masks together and thresholding at four. 


\section{$\underline{\text { Discussion }}$}

In our three consecutive investigations the visual system was examined starting from neuronal level using single-unit recording up to the whole brain network interaction by diffusion tractography. In the animal experiment, we have reported an aging related increase in response latency and a worsening of the stimulus selectivity in awake, behaving monkeys. The investigation of motion detection revealed local correlations between the white matter microstructure parameters measured by diffusion MRI and the coherent motion detection performance in several regions that can be linked to motion detection and attention functions. In the experiment of audio-visual integration, we evoked a robust double-flash illusion through the dorsal and ventral visual streams and the behavioural data correlated well with the microstructure of the two relevant visual white matter pathways.

\section{Aging effects on object vision in monkeys}

Macaque monkeys have been a widely used animal model to study human visual perception. Several papers have compared the homology to humans; the investigation of changes in the shape representation of the monkey IT may therefore facilitate an understanding of age-related changes in the human visual system.

\section{Increased latency by ageing}

In the IT, on the average latency values are about 100 ms (Schmolesky et al., 1998), however, values around $150 \mathrm{~ms}$ are not extreme (Tamura and Tanaka, 2001). Poisson spike train analysis (Hanes et al., 1995) was used, because that algorithm can detect little variance in the neuronal response latency time (Sary et al., 2006). Nevertheless we measured increased latencies in the IT in older monkeys. It is not possible to explain the origin of the delayed latencies from the data we report here. One possible reason is a general slowing in the speed of processing year after year (Salthouse, 1996). Such aging related delay was reported in rats (Wang et al., 2006). Similarly, it was shown in humans that the latency of the P300 component is delayed with aging (Morgan and Murphy, 2010). Likewise, cognitively high and average performers produced higher latency values with ages (Riis et al., 2008), which indicates the age-dependent factors in visual processing during normal aging. Face matching task in a PET study showed increased 
reaction times (Grady et al., 1994). The underlying mechanism is still debated, since the number of retinal ganglion cells (Kim et al., 1996) or the number of the dorsal lateral geniculate body cells (dLGB) (Ahmad and Spear, 1993) are not decreasing during aging. Although degradation was detected in layer 3 of V1, that did not affect the electrophysiological properties (Luebke et al., 2013), and no relevant deviation was accessed in the total numbers of V1 neurons and glial cells (Giannaris and Rosene, 2012). It was recently suggested, that myelin breakdown could have an influence on the speed of processing ( $\mathrm{Lu}$ et al., 2011; Lu et al., 2013), and we hypothesize that this might be the case behind the increased response latencies observed. It was confirmed that a strong correlation exists between age-related myelin breakdown and cognitive impairment in monkeys (Peters and Sethares, 2002). Furthermore, in aged cats, the axonal conduction velocity is slowed down (Xi et al., 1999). Recently, it is suggested that in the cortex, the age-associated deterioration of the myelin and electrophysiological parameters change together (Luebke et al., 2010). This slowing could be a general phenomenon in the nervous system, but it certainly affects the corresponding visual cortical areas as well (Peters et al., 2000).

\section{Decreased stimulus selectivity}

The cells in IT respond well to complex objects, but with different firing rates. Stimulus preference is the basic property of these neurons, i.e., a distinction between preferred and non-preferred stimuli by firing rate. The recent hypothesis of object coding includes a limited number of cortical modules and cells with different preference (Desimone et al., 1984; Gross et al., 1972; Tanaka et al., 1991). In this way, the coding of the viewed stimuli depends on the firing pattern of a group of cells. Local neurones produce a population code, which consist of the combination of active/inactive cortical columns and a combination of active/inactive cells within the column. The key to the perception of different aspects of the visual stimulus thus is probably a set of neurons with stimulus selectivity (or more precisely, stimulus preference) for a particular parameter (speed, direction, orientation, shape, colour, etc.), and thus an altered preference or age induced influence on the firing pattern of these neurons will have an impact on the perception of the particular item (Salzman and Newsome, 1994).

Experiments focused on the neuronal operation showed, that in the level of dorsal LGB, there is a slight, functional, age-related alteration (Spear et al., 1994), and through the visual hierarchy, in higher stages there is a larger degree of signal degradation 
(Schmolesky et al., 2000). V1 is less affected than the V2 (Yu et al., 2006) likewise the higher levels of visual perception (Liang et al., 2010). Although, some age-related gap exists between the simple and complex cells in V1 as regarding age-related deteriorations (Liang et al., 2012). Human investigations showed evidence in changes in shape perception (McKendrick et al., 2010; Weymouth and McKendrick, 2012) and also in visuo-spatial memory (Carp et al., 2010).

A number of studies proposed a structural deterioration of the neuronal mechanisms supporting visual perception. In the MT of monkeys, age-related alteration of speed representation and the increase in response variability were found. The possible underlying mechanism could be the lower number of glycinergic neurons (Yang et al., 2009a; Yang et al., 2009b) and also the decrease in the number of interneurons in the cortex (Coleman and Flood, 1987). The receptive field of IT cells is shaped by GABAergic neurons (Wang et al., 2002). It is possible, that with years a degradation of these cells (in number or in function) could cause changes in shape processing. In addition, aging led to disintegration of myelin sheets (Inano et al., 2011) and alteration in fractional anisotropy in the occipital lobe (Van Impe et al., 2012), which was suggested to be a potential biomarker of sensory degeneration. Furthermore, in auditory and visual cortices an age related aggregation of microglias and oligodendrocytes was observed (Tremblay et al., 2012). Another possible solution could be the reduction of the occipital grey matter and a decrease also in the activity with the impairment of sensory-perceptual processing (Kalpouzos et al., 2012). Similarly, recently it was showed that decreased discrimination capability is related to decreased grey matter volume by aging (Carp et al., 2010). Assumptions state that perceptual impairments in ageing are due to functional rather than morphological changes, which affect the communication between neurons (Hua et al., 2006; Leventhal et al., 2003; Mendelson and Wells, 2002; Schmolesky et al., 2000; Wang et al., 2006; Yang et al., 2008). Unfortunately our findings do not allow an identification of the mechanisms mentioned above; we have merely demonstrated a phenomenon that has previously not been described.

In summary, we have reported an increase in response latency and a worsening of the stimulus selectivity in the monkey homolog of the human LOC, which might explain, at least partially the uncertainties in visual perception observed in the elderly. 


\section{Attention modulation of motion perception}

Previous studies (Dubner and Zeki, 1971; Tootell et al., 1995; Zeki et al., 1991; Zihl et al., 1983) revealed the relevant motion-sensitive visual areas in the dorsal visual stream, around the occipital-temporal-parietal axis. One of the most relevant parts of this system is the V5/MT+ complex. In our study, the systematic analysis showed that the motion detection threshold is closely linked to the white matter microstructure in the pathway related to attentional activity functions. Presumably, during the observation of the coherent motion in random noise, the task requires such attention, therefore stronger attention leads to better performance. Attentional modulation of the extrastriate areas in motion detection task was shown formerly (Buchel et al., 1998; Kellermann et al., 2012). The main areas of this modulation are found in the frontal and parietal regions. In the seminal paper of Buchel, similar random-dot kinematogram was used and areas related to attention to visual motion were defined (Buchel et al., 1998). The identified areas in the frontal eye field, the premotor cortex and the posterior parietal cortex demonstrated a high level of correspondence with the cortical regions to where the white matter regions projected in our analysis. A recent hypothesis suggested the existence of two segregated attention systems (Corbetta and Shulman, 2002). The dorsal attention network, responsible for goal-directed (top-down) attentional modulation, which includes parts of the dorsal parietal cortex (the superior parietal lobule and the intraparietal sulcus) and the superior frontal cortex (near or at the frontal eye field - FEF); whereas the ventral network driven by salient stimuli (bottom-up modulation), taking place in the temporoparietal junction and the inferior frontal cortex. The bottom-up orienting function proved to be negatively correlated with the cortical thickness in the angular gyrus and the superior parietal lobule and the mean diffusivity under the inferior parietal lobe (Yin et al., 2012). In contrast, the alteration of top-down attention functions was correlated with the FA in the superior frontal region (the white matter under the frontal-eye field, similarly as found in our analysis)(Yin et al., 2012). Interestingly, our analysis found both the dorsal and ventral attention systems to be active during this task. This is not surprising, since continuous attention is directed to the moving dot pattern during the experiment and the ventral salience system might also be activated when the coherent motion pattern is finally detected. Recent finding showed that within the superior longitudinal fascicle separated fibres attend in the two attention networks (Thiebaut de Schotten et al., 2011). The dorsal fascicle takes part in the dorsal attention network, while 
the ventral ones in the ventral attention network. The third take place in both, which can be the nexus of the two systems, with switching function of the segregated attention systems (Corbetta and Shulman, 2002; Thiebaut de Schotten et al., 2011). Newer evidence (Gruber et al., 2010) and an integrative theory of mind (Abu-Akel and ShamayTsoory, 2011) support the relevance of this interference, which modulation centre could be the temporo-parietal junction as shifting of mental states and orientation. Another possible solution is V5/MT+ reaction relates to the dorsal/ventral interactions (Caclin et al., 2012). Recent studies discovered the importance of the temporo-parietal junction in both attention systems (Chica et al., 2011). Attentional modulation in V5/MT+ was presented in former studies (Buchel et al., 1998; O'Craven et al., 1997; Treue and Maunsell, 1996). Along the same lines in functional and effective connectivity investigations, it was proposed that attentional modulation originate from the parietal and frontal areas (Buchel and Friston, 1997; Marreiros et al., 2008). Functional connectivity studies have reflected the feasible areas of attentional modulation (Buchel and Friston, 1997; Friston et al., 1997; Penny et al., 2004). Two-state dynamic causal modelling proposed that the alteration of the forward connections from V1 to V5 is essential in attention to visual motion (Marreiros et al., 2008). Our findings are somewhat controversial. The performance in the attention-demanding motion detection task correlated well with the integrity of the white matter tracts within the attention networks rather than with those pathways connecting the attention networks to the visual regions or connecting various visual areas. This could suggest that the bottom line of the performance in the motion detection task is the "structural integrity" of the attentional networks.

In conclusion, examining the white matter background of motion detection, we revealed the main components of the attention networks that are related to the random-dot kinematogram paradigm. Similarly, this study highlights the importance of the structural determinants of perception. Hopefully, the presented results promote to apprehend the processing of motion detection, and, in general, the visual sensation, and in future all together assist to better representation and interpretation of attention-related alteration in several diseases. 


\section{Double-flash illusion on the segregated visual streams}

Previous finding indicated the involvement of the ventral pathway in audio-visual integration, as Jaekl and Harris during a metacontrast masking paradigm got around the inhibitory effect of M-pathway-related transient activity on the P-pathway with concurrent auditory stimulus which elevated the 'What' stream activity (Jaekl and Harris, 2009). Additionally, in an orientation discrimination task where appropriate timed auditory stimuli could modulated the threshold, similar conclusion was reached (Jaekl and Harris, 2009). Chromatic short-wavelength stimuli with concurrent auditory stimulus altered the reaction time (Leo et al., 2008). On the other hand, bimodal integration is shown also in the dorsal, in the 'Where'-stream. Auditory information in a contrast sensitivity task in a steady, but not in a pulsed-pedestal condition altered, meaning that dorsal, but not ventral pathway was involved (Jaekl and Soto-Faraco, 2010). Similarly, the same finding was revealed as interaction occurred in lower, but not in higher spatial frequencies (Jaekl and Soto-Faraco, 2010). Equally, in an audio-visual fMRI task, the 'Where' pathway specific stimuli showed the relevance of the identical stream (Watkins et al., 2006; Watkins et al., 2007). Though our findings are somewhat controversial, we have to mention, that no stimuli exists, which would be processed absolutely segregated on the visual streams, but our stimuli in this study were created in order to drive preferentially the magno- or the parvocellular pathways, respetively (Kaposvari et al., 2014).

The underlying mechanisms of interaction of different sensory modalities are still under an intense research. One solution is the presence of long-range disinhibited feedback like in the case of illusions and hallucinations by psychedelic drugs. Second possibility is the cross-activation of brain regions. Other is the existence of re-entry processing while the information is circulating among the neuroanatomical regions involved. Another issue is the hyperbinding connectivity under parietal modulation based on binding theory. The last and maybe the best opportunity is the multiple neural model where all the previously mentioned forms are appearing together (Hubbard, 2007).

Despite the missing pieces of the complete picture to understand the cross-modal interaction and the network-level based perception it is important to note its' biological relevance. From the first primitive animals to human beings, the information processing became more complex that increased the chances of survival of species making it more difficult to sort and integrate the information from the outside world at the same time. 
During a multisensory task synchrony occurs among vision, touch and audition (etc.) (Vroomen and Keetels, 2010) where non-visual stimuli qualitatively and quantitatively modify the cross-modal integration (Shams and Kim, 2010) and make the perception sharper or smarter (Jaekl et al., 2014). Audio-visual enhancements caught out as decrease in response latencies (Corneil et al., 2002) and visual search times (Van der Burg et al., 2008), speed up motion detection (Meyer et al., 2005) and lower the detection threshold (Gleiss and Kayser, 2013) nevertheless modulate the information content (McGurk and MacDonald, 1976). It is hypothesized that these effects originate from the magnocellular pathway or an intense interaction between the two visual streams can be found behind these effects (which is highly possible based on our findings).

Summarily, in this study, correlation was observed between the white matter microstructure parameters and the likelihood of perceiving double-flash illusion using stimuli parameter preferred by the segregated visuals streams that suggest the fact that audio-visual integration involves systems. Up to our knowledge, this is the first study that showed the white matter microstructural background of the segregated, the so-called 'What' and 'Where' visual pathways.

\section{Structure- function relationship}

Finally, it is worth mentioning the relationship between structure and function. Neuroimaging studies basically detect cumulative activation or morphological alterations of larger cell clusters. At the same time, newly developed specific MRI sequences and tools are available, which directly focus on the gray matter (voxel-based morphology (VBM) style analysis) or white matter (DTI) specific variations in lower scale.

The previously mentioned study of the taxi drivers' hippocampus was the first of the grey matter density changes related to performance (Maguire et al., 2000). Similarly, musicians' auditory and motor cortex volume and thickness is much larger than in the control group (Bermudez et al., 2009). Cortical expansion was observed as longitudinal effect of learning to juggle in the motion detection area of the visual system (Draganski et al., 2004b). Similarly, the white matter is under intense examinations, as how the white matter integrity and structural connectivity is related to brain functions. Variability in brain function was shown to be determined by the variation of the connectivity profile in certain regions (Behrens et al., 2006; Johansen-Berg et al., 2004; Klein et al., 2007; Saygin et al., 2012). Behavioural performance is related to the locally measured diffusion 
profile of the white matter (Johansen-Berg, 2010). Better bimanual coordination is related to higher FA in the corpus callosum (Johansen-Berg et al., 2007). The elevation in FA in the intraparietal sulcus was found to correlate with training (Scholz et al., 2009). The mean diffusivity of the hippocampus enhanced during rapid, within hours spatial learning task (Sagi et al., 2012). Similarly, in the occipital cortex, higher BOLD response was related to greater FA in the optic radiation (Toosy et al., 2004). Recently, combined neuroimaging and histological approaches showed that learning (spatial, motor) is related to the locally enhanced myelination that is represented by enhanced FA (BlumenfeldKatzir et al., 2011; Sampaio-Baptista et al., 2013). Commonly, increase in FA is related to better function but sometimes, like our motion detection study, the effect is inversed. The background mechanism of the FA - performance relation is under an intense investigation. The hypotheses suggest that the element of cortical plasticity such as dendritic arborisation, glial changes or angiogenesis play an important role (Zatorre et al., 2012). Enhance in fractional anisotropy is caused by fibres reorganisation, change in myelin-formation, oligodendrocites occurrence and increase in myelin-shield thickness (Lerch et al., 2011; Wedeen et al., 2005).

The nexus between the functional and structural connectivity (Parks and Madden, 2013) is also highly investigated. It was suggested, that higher myelination is related to higher functional connectivity (Giedd et al., 1999). In the case of the cingulum, the functional connectivity was positively correlated with the locally measured FA (van den Heuvel et al., 2008), while these results should be interpreted with caution (Damoiseaux and Greicius, 2009). It is still questionable, what is the underlying mechanism of the coupling between the histological properties and the diffusion parameter of the white matter. It is a well known fact that the conduction velocity and dispersion of the neuronal signal is in closely related to the diameter of nerves and myelination (Hodgkin and Huxley, 1952), which related to the diffusion tensor imaging . 


\section{$\underline{\text { Summary }}$}

This thesis report aims to investigate two main fields. First, the structural determinants of behaviour and second, the deterioration of the neuronal response by ageing. It was presented on awake, behaving monkeys that stimulus selectivity is decreased and the neuronal response latency is increased with aging. Diffusion MRI investigations showed correlation between the white matter microstructure parameter and the behavioural performance. In the task of motion detection, the attentional networks were highlighted. Furthermore, it was suggested that the double-flash illusion involves both members of the segregated visual streams.

\section{$\underline{\text { Acknowledgements }}$}

I would like to express my grateful thanks to those who helped my work and to finish this dissertation. I would like to thank Professor László Vécsei for giving me the opportunity to work in the Department of Neurology. I would like to express my gratitude to my tutor, Dr. Zsigmond Tamás Kincses for his kind support and valuable guidance. I would like to say thanks to Professor Gyula Sáry who gave me advices in science and in life. Special thanks goes Nikoletta Szabó, Péter Kaposvári, Eszter Tóth, Bernadett Tuka, Rita Török, Gábor Veres, Viola Luca Németh, Péter Csibri, Anna Bognár, András Király, Péter Faragó, Krisztián Kocsis and all the current and former member of the Neuroimaging Research Group and Visual Laboratory for their wonderful collaboration. I would like to give my special thanks to Judit Tóth whose support, love and patience enabled me to complete this work. Also I would like to give my thanks to my family and my friends who support and encourage me.

This research was supported by the European Union and the State of Hungary, cofinanced by the European Social Fund in the framework of TÁMOP-4.2.4.A/2-11/12012-0001 'National Excellence Program'. Gergő Csete was supported by the 'Asthellas Young Talent Program' (Asthellas Pharma Inc, Szeged, 2015). 


\section{$\underline{\text { References }}$}

Abu-Akel, A., Shamay-Tsoory, S., 2011. Neuroanatomical and neurochemical bases of theory of mind. Neuropsychologia. 49, 2971-84.

Afraz, S.R., Kiani, R., Esteky, H., 2006. Microstimulation of inferotemporal cortex influences face categorization. Nature. 442, 692-5.

Ahmad, A., Spear, P.D., 1993. Effects of aging on the size, density, and number of rhesus monkey lateral geniculate neurons. J Comp Neurol. 334, 631-43.

Allred, S., Liu, Y., Jagadeesh, B., 2005. Selectivity of inferior temporal neurons for realistic pictures predicted by algorithms for image database navigation. $\mathbf{J}$ Neurophysiol. 94, 4068-81.

Andersen, G.J., 2012. Aging and Vision: Changes in Function and Performance from Optics to Perception. Wiley Interdiscip Rev Cogn Sci. 3, 403-410.

Angelucci, A., et al., 2002. Circuits for local and global signal integration in primary visual cortex. J Neurosci. 22, 8633-46.

Antal, A., et al., 2005. Altered motion perception in migraineurs: evidence for interictal cortical hyperexcitability. Cephalalgia. 25, 788-94.

Baleydier, C., Morel, A., 1992. Segregated thalamocortical pathways to inferior parietal and inferotemporal cortex in macaque monkey. Vis Neurosci. 8, 391-405.

Barker, A.T., Jalinous, R., Freeston, I.L., 1985. Non-invasive magnetic stimulation of human motor cortex. Lancet. 1, 1106-7.

Bates, E., et al., 2003. Voxel-based lesion-symptom mapping. Nat Neurosci. 6, 448-50.

Beadsmoore, C., et al., 2015. Positron Emission Tomography Computed Tomography: A Guide for the General Radiologist. Can Assoc Radiol J.

Beckett, A., et al., 2012. Contribution of large scale biases in decoding of direction-ofmotion from high-resolution fMRI data in human early visual cortex. Neuroimage. 63, 1623-1632.

Behrens, T.E., et al., 2006. A consistent relationship between local white matter architecture and functional specialisation in medial frontal cortex. Neuroimage. 30, 220-7.

Behrens, T.E., et al., 2007. Probabilistic diffusion tractography with multiple fibre orientations: What can we gain? Neuroimage. 34, 144-55.

Bennett, P.J., Sekuler, R., Sekuler, A.B., 2007. The effects of aging on motion detection and direction identification. Vision Res. 47, 799-809.

Berger, H., 1929. Über das Elektrenkephalogramm des Menschen. Archiv für Psychiatrie und Nervenkrankheiten. 87, 527-570.

Bermudez, P., et al., 2009. Neuroanatomical correlates of musicianship as revealed by cortical thickness and voxel-based morphometry. Cereb Cortex. 19, 1583-96.

Betts, L.R., Sekuler, A.B., Bennett, P.J., 2007. The effects of aging on orientation discrimination. Vision Res. 47, 1769-80.

Bhattacharya, J., Shams, L., Shimojo, S., 2002. Sound-induced illusory flash perception: role of gamma band responses. Neuroreport. 13, 1727-30.

Blumenfeld-Katzir, T., et al., 2011. Diffusion MRI of structural brain plasticity induced by a learning and memory task. PLoS One. 6, e20678.

Bondar, I.V., et al., 2009. Long-term stability of visual pattern selective responses of monkey temporal lobe neurons. PLoS One. 4, e8222.

Born, R.T., Bradley, D.C., 2005. Structure and function of visual area MT. Annu Rev Neurosci. 28, 157-89. 
Boulton, A.A., Baker, G.B., Vanderwolf, C.H., 1990. Neurophysiological Techniques: Applications to Neural Systems, Vol., Humana Press.

Boussaoud, D., Ungerleider, L.G., Desimone, R., 1990. Pathways for motion analysis: cortical connections of the medial superior temporal and fundus of the superior temporal visual areas in the macaque. J Comp Neurol. 296, 462-95.

Brannan, J.R., Bodis-Wollner, I., 1991. Evidence for two systems mediating perceived contrast. Vis Neurosci. 6, 587-92.

Braunitzer, G., et al., 2012. Delayed development of visual motion processing in childhood migraine. Cephalalgia. 32, 492-6.

Broca, P.P., 1861a. Remarques sur le siège de la faculté du langage articulé, suivies d'une observation d'aphémie (perte de la parole).

Broca, P.P., 1861b. Nouvelle observation d'aphémie produite par une lésion de la moitié postérieure des deuxième et troisième circonvolutions frontales.

Buchel, C., Friston, K.J., 1997. Modulation of connectivity in visual pathways by attention: cortical interactions evaluated with structural equation modelling and fMRI. Cereb Cortex. 7, 768-78.

Buchel, C., et al., 1998. The functional anatomy of attention to visual motion. A functional MRI study. Brain. 121 ( Pt 7), 1281-94.

Bucy, P.C., Kluver, H., 1955. An anatomical investigation of the temporal lobe in the monkey (Macaca mulatta). J Comp Neurol. 103, 151-251.

Caclin, A., et al., 2012. Perceptual alternations between unbound moving contours and bound shape motion engage a ventral/dorsal interplay. J Vis. 12.

Carp, J., Gmeindl, L., Reuter-Lorenz, P.A., 2010. Age differences in the neural representation of working memory revealed by multi-voxel pattern analysis. Front Hum Neurosci. 4, 217.

Carter, S.E., et al., 1997. Environmental hazards in the homes of older people. Age Ageing. 26, 195-202.

Chawla, D., et al., 1998. Speed-dependent motion-sensitive responses in V5: an fMRI study. Neuroimage. 7, 86-96.

Chee, M.W.L., et al., 2006. Age-related changes in object processing and contextual binding revealed using fMR adaptation. Journal of Cognitive Neuroscience. 18, 495-507.

Chica, A.B., Bartolomeo, P., Valero-Cabre, A., 2011. Dorsal and ventral parietal contributions to spatial orienting in the human brain. J Neurosci. 31, 8143-9.

Churchland, M.M., et al., 2010. Stimulus onset quenches neural variability: a widespread cortical phenomenon. Nat Neurosci. 13, 369-78.

Cohen, D., 1968. Magnetoencephalography: evidence of magnetic fields produced by alpha-rhythm currents. Science. 161, 784-6.

Coleman, P.D., Flood, D.G., 1987. Neuron numbers and dendritic extent in normal aging and Alzheimer's disease. Neurobiol Aging. 8, 521-45.

Corbetta, M., Shulman, G.L., 2002. Control of goal-directed and stimulus-driven attention in the brain. Nat Rev Neurosci. 3, 201-15.

Corneil, B.D., et al., 2002. Auditory-visual interactions subserving goal-directed saccades in a complex scene. J Neurophysiol. 88, 438-54.

Damoiseaux, J.S., Greicius, M.D., 2009. Greater than the sum of its parts: a review of studies combining structural connectivity and resting-state functional connectivity. Brain Structure and Function. 213, 525-533.

Denys, K., et al., 2004. The processing of visual shape in the cerebral cortex of human and nonhuman primates: a functional magnetic resonance imaging study. $\mathrm{J}$ Neurosci. 24, 2551-65. 
Desapriya, E., et al., 2014. Vision screening of older drivers for preventing road traffic injuries and fatalities. Cochrane Database Syst Rev. 2, CD006252.

Desimone, R., et al., 1984. Stimulus-selective properties of inferior temporal neurons in the macaque. J Neurosci. 4, 2051-62.

DeYoe, E.A., et al., 1996. Mapping striate and extrastriate visual areas in human cerebral cortex. Proc Natl Acad Sci U S A. 93, 2382-2386.

Draganski, B., et al., 2004a. Neuroplasticity: changes in grey matter induced by training. Nature. 427, 311-2.

Draganski, B., et al., 2004b. Neuroplasticity: Changes in grey matter induced by training - Newly honed juggling skills show up as a transient feature on a brain-imaging scan. Nature. 427, 311-312.

Dubner, R., Zeki, S.M., 1971. Response properties and receptive fields of cells in an anatomically defined region of the superior temporal sulcus in the monkey. Brain Res. 35, 528-32.

Fechner, G.T., 1860. Elemente der Psychophysik, Vol., Breitkopf und Härtel.

Felleman, D.J., Van Essen, D.C., 1991. Distributed hierarchical processing in the primate cerebral cortex. Cereb Cortex. 1, 1-47.

Fernandes, A., et al., 2003. Ocular measurements throughout the adult life span of rhesus monkeys. Invest Ophthalmol Vis Sci. 44, 2373-2380.

Fize, D., et al., 2003. The retinotopic organization of primate dorsal V4 and surrounding areas: A functional magnetic resonance imaging study in awake monkeys. J Neurosci. 23, 7395-406.

Floel, A., et al., 2009. White matter integrity in the vicinity of Broca's area predicts grammar learning success. Neuroimage. 47, 1974-81.

Friston, K.J., et al., 1997. Psychophysiological and modulatory interactions in neuroimaging. Neuroimage. 6, 218-29.

Gallagher, M., Rapp, P.R., 1997. The use of animal models to study the effects of aging on cognition. Annual Review of Psychology. 48, 339-370.

Gennari, F., 1782. De peculli structura cerebri nonnulisque ejus morbis, Vol., Parma.

Giannaris, E.L., Rosene, D.L., 2012. A stereological study of the numbers of neurons and glia in the primary visual cortex across the lifespan of male and female rhesus monkeys. J Comp Neurol. 520, 3492-508.

Giedd, J.N., et al., 1999. Brain development during childhood and adolescence: a longitudinal MRI study. Nat Neurosci. 2, 861-3.

Gleiss, S., Kayser, C., 2013. Eccentricity dependent auditory enhancement of visual stimulus detection but not discrimination. Front Integr Neurosci. 7, 52.

Grady, C.L., et al., 1994. Age-related changes in cortical blood flow activation during visual processing of faces and location. J Neurosci. 14, 1450-62.

Gross, C.G., Rocha-Miranda, C.E., Bender, D.B., 1972. Visual properties of neurons in inferotemporal cortex of the Macaque. J Neurophysiol. 35, 96-111.

Gruber, O., et al., 2010. A neural system for evaluating the behavioural relevance of salient events outside the current focus of attention. Brain Res. 1351, 212-21.

Habak, C., Wilkinson, F., Wilson, H.R., 2009. Preservation of shape discrimination in aging. J Vis. 9, 18 1-8.

Hanes, D.P., Thompson, K.G., Schall, J.D., 1995. Relationship of presaccadic activity in frontal eye field and supplementary eye field to saccade initiation in macaque: Poisson spike train analysis. Exp Brain Res. 103, 85-96.

Harlow, 1848. Passage of an Iron Rod through the Head. The Boston Medical and Surgical Journal. 39, 389-393. 
Haxby, J.V., et al., 1991. Dissociation of object and spatial visual processing pathways in human extrastriate cortex. Proc Natl Acad Sci U S A. 88, 1621-5.

Hodgkin, A.L., Huxley, A.F., 1952. Propagation of electrical signals along giant nerve fibers. Proc R Soc Lond B Biol Sci. 140, 177-83.

Hua, T., et al., 2006. Functional degradation of visual cortical cells in old cats. Neurobiol Aging. 27, 155-62.

Hubbard, E.M., 2007. Neurophysiology of synesthesia. Curr Psychiatry Rep. 9, 193-9.

Hubel, D.H., Wiesel, T.N., 1959. Receptive fields of single neurones in the cat's striate cortex. J Physiol. 148, 574-91.

Hubel, D.H., Wiesel, T.N., 1962. Receptive fields, binocular interaction and functional architecture in the cat's visual cortex. J Physiol. 160, 106-54.

Hubel, D.H., Wiesel, T.N., 1968. Receptive fields and functional architecture of monkey striate cortex. J Physiol. 195, 215-43.

Hutman, L.P., Sekuler, R., 1980. Spatial vision and aging. II: Criterion effects. J Gerontol. 35, 700-6.

Inano, S., et al., 2011. Effects of age and gender on white matter integrity. AJNR Am J Neuroradiol. 32, 2103-9.

Jaekl, P., Pérez-Bellido, A., Soto-Faraco, S., 2014. On the 'visual' in 'audio-visual integration': a hypothesis concerning visual pathways. Experimental Brain Research. 232, 1631-1638.

Jaekl, P.M., Harris, L.R., 2009. Sounds can affect visual perception mediated primarily by the parvocellular pathway. Vis Neurosci. 26, 477-86.

Jaekl, P.M., Soto-Faraco, S., 2010. Audiovisual contrast enhancement is articulated primarily via the M-pathway. Brain Res. 1366, 85-92.

Jenkinson, M., et al., 2012. Fsl. Neuroimage. 62, 782-90.

Johansen-Berg, H., et al., 2004. Changes in connectivity profiles define functionally distinct regions in human medial frontal cortex. Proc Natl Acad Sci U S A. 101, 13335-40.

Johansen-Berg, H., et al., 2007. Integrity of white matter in the corpus callosum correlates with bimanual co-ordination skills. Neuroimage. 36 Suppl 2, T16-21.

Johansen-Berg, H., Behrens, T.E.J., 2009. Diffusion MRI: From quantitative measurement to in-vivo neuroanatomy, Vol., Elsevier Science.

Johansen-Berg, H., 2010. Behavioural relevance of variation in white matter microstructure. Curr Opin Neurol. 23, 351-8.

Judge, S.J., Richmond, B.J., Chu, F.C., 1980. Implantation of magnetic search coils for measurement of eye position: an improved method. Vision Res. 20, 535-8.

Kallstrand-Eriksson, J., et al., 2013. Perceived vision-related quality of life and risk of falling among community living elderly people. Scandinavian Journal of Caring Sciences. 27, 433-439.

Kalpouzos, G., Persson, J., Nyberg, L., 2012. Local brain atrophy accounts for functional activity differences in normal aging. Neurobiol Aging. 33, 623 e1-623 e13.

Kaposvari, P., et al., 2014. Fusion and fission in the visual pathways. Physiol Res.

Kellermann, T., et al., 2012. Effective connectivity of the human cerebellum during visual attention. J Neurosci. 32, 11453-60.

Kim, C.B., Tom, B.W., Spear, P.D., 1996. Effects of aging on the densities, numbers, and sizes of retinal ganglion cells in rhesus monkey. Neurobiol Aging. 17, 431-8.

Kincses, Z.T., et al., 2011. Lesion probability mapping to explain clinical deficits and cognitive performance in multiple sclerosis. Mult Scler. 17, 681-9.

Kincses, Z.T., et al., 2014. Grey matter atrophy in patients suffering from multiple sclerosis. Ideggyogy Sz. 67, 293-300. 
Klein, J.C., et al., 2007. Connectivity-based parcellation of human cortex using diffusion MRI: Establishing reproducibility, validity and observer independence in BA 44/45 and SMA/pre-SMA. Neuroimage. 34, 204-11.

Kourtzi, Z., et al., 2003. Integration of local features into global shapes: monkey and human FMRI studies. Neuron. 37, 333-346.

Kuba, M., et al., 2012. Aging effect in pattern, motion and cognitive visual evoked potentials. Vision Res. 62, 9-16.

Kuffler, S.W., 1953. Discharge patterns and functional organization of mammalian retina. J Neurophysiol. 16, 37-68.

Landi, S.M., Baguear, F., Della-Maggiore, V., 2011. One Week of Motor Adaptation Induces Structural Changes in Primary Motor Cortex That Predict Long-Term Memory One Year Later. Journal of Neuroscience. 31, 11808-11813.

Larsson, J., Heeger, D.J., Landy, M.S., 2010. Orientation selectivity of motion-boundary responses in human visual cortex. J Neurophysiol. 104, 2940-50.

Legge, G.E., 1978. Sustained and transient mechanisms in human vision: temporal and spatial properties. Vision Res. 18, 69-81.

Leo, F., et al., 2008. Multisensory integration for orienting responses in humans requires the activation of the superior colliculus. Exp Brain Res. 186, 67-77.

Leopold, D.A., Logothetis, N.K., 1996. Activity changes in early visual cortex reflect monkeys' percepts during binocular rivalry. Nature. 379, 549-53.

Lerch, J.P., et al., 2011. Maze training in mice induces MRI-detectable brain shape changes specific to the type of learning. Neuroimage. 54, 2086-95.

Leventhal, A.G., et al., 2003. GABA and its agonists improved visual cortical function in senescent monkeys. Science. 300, 812-5.

Lewis, J.W., Van Essen, D.C., 2000. Corticocortical connections of visual, sensorimotor, and multimodal processing areas in the parietal lobe of the macaque monkey. $\mathbf{J}$ Comp Neurol. 428, 112-37.

Liang, Z., et al., 2010. Aging affects the direction selectivity of MT cells in rhesus monkeys. Neurobiol Aging. 31, 863-73.

Liang, Z., et al., 2012. Selective effects of aging on simple and complex cells in primary visual cortex of rhesus monkeys. Brain Res. 1470, 17-23.

Logothetis, N.K., et al., 2001. Neurophysiological investigation of the basis of the fMRI signal. Nature. 412, 150-157.

Lowenstine, L.J., 2003. A primer of primate pathology: lesions and nonlesions. Toxicol Pathol. 31 Suppl, 92-102.

Lu, F.M., Yuan, Z., 2015. PET/SPECT molecular imaging in clinical neuroscience: recent advances in the investigation of CNS diseases. Quant Imaging Med Surg. 5, 43347.

Lu, P.H., et al., 2011. Age-related slowing in cognitive processing speed is associated with myelin integrity in a very healthy elderly sample. Journal of Clinical and Experimental Neuropsychology. 33, 1059-1068.

Lu, P.H., et al., 2013. Myelin breakdown mediates age-related slowing in cognitive processing speed in healthy elderly men. Brain and Cognition. 81, 131-138.

Luck, S.J., Kappenman, E.S., 2012. The Oxford Handbook of Event-Related Potential Components, Vol., Oxford University Press, USA.

Luebke, J., Barbas, H., Peters, A., 2010. Effects of normal aging on prefrontal area 46 in the rhesus monkey. Brain Research Reviews. 62, 212-232.

Luebke, J.I., et al., 2013. Age-Related Changes to Layer 3 Pyramidal Cells in the Rhesus Monkey Visual Cortex. Cereb Cortex. 
Lui, L.L., Rosa, M.G., 2015. Structure and function of the middle temporal visual area (MT) in the marmoset: Comparisons with the macaque monkey. Neurosci Res. 93, 62-71.

MacMillan, N., Creelman, C., 2005. Detection Theory: A User's Guide Routledge, Vol., Psychology Press.

Maguire, E.A., et al., 2000. Navigation-related structural change in the hippocampi of taxi drivers. Proc Natl Acad Sci U S A. 97, 4398-403.

Marreiros, A.C., Kiebel, S.J., Friston, K.J., 2008. Dynamic causal modelling for fMRI: a two-state model. Neuroimage. 39, 269-78.

Maunsell, J.H., van Essen, D.C., 1983. The connections of the middle temporal visual area (MT) and their relationship to a cortical hierarchy in the macaque monkey. $\mathbf{J}$ Neurosci. 3, 2563-86.

McGurk, H., MacDonald, J., 1976. Hearing lips and seeing voices. Nature. 264, 746-8.

McKendrick, A.M., Weymouth, A.E., Battista, J., 2010. The effect of normal aging on closed contour shape discrimination. J Vis. 10, 1 1-9.

Mendelson, J.R., Wells, E.F., 2002. Age-related changes in the visual cortex. Vision Res. 42, 695-703.

Merigan, W.H., 1996. Basic visual capacities and shape discrimination after lesions of extrastriate area V4 in macaques. Vis Neurosci. 13, 51-60.

Meyer, G.F., et al., 2005. Low-level integration of auditory and visual motion signals requires spatial co-localisation. Exp Brain Res. 166, 538-47.

Mishra, J., et al., 2007. Early cross-modal interactions in auditory and visual cortex underlie a sound-induced visual illusion. J Neurosci. 27, 4120-31.

Missal, M., et al., 1999. Shape interactions in macaque inferior temporal neurons. J Neurophysiol. 82, 131-42.

Montez, T., et al., 2009. Altered temporal correlations in parietal alpha and prefrontal theta oscillations in early-stage Alzheimer disease. Proc Natl Acad Sci U S A. 106, 1614-9.

Morel, A., Bullier, J., 1990. Anatomical segregation of two cortical visual pathways in the macaque monkey. Vis Neurosci. 4, 555-78.

Morgan, C.D., Murphy, C., 2010. Differential effects of active attention and age on eventrelated potentials to visual and olfactory stimuli. Int J Psychophysiol. 78, 190-9.

Morrone, M.C., et al., 2000. A cortical area that responds specifically to optic flow, revealed by fMRI. Nat Neurosci. 3, 1322-8.

Mosso, A., 1881. Ueber den kreislauf des blutes im menschlichen gehirn: untersuchungen, Vol., Verlag von Veit \& Comp.

Nichols, T.E., Holmes, A.P., 2002. Nonparametric permutation tests for functional neuroimaging: a primer with examples. Hum Brain Mapp. 15, 1-25.

O'Craven, K.M., et al., 1997. Voluntary attention modulates fMRI activity in human MTMST. Neuron. 18, 591-8.

Ogawa, S., et al., 1990. Brain magnetic resonance imaging with contrast dependent on blood oxygenation. Proc Natl Acad Sci U S A. 87, 9868-72.

Ooi, C.S., Grosvenor, T., 1995. Mechanisms of emmetropization in the aging eye. Optom Vis Sci. 72, 60-6.

Ooj, C.S., Grosvenor, T., 1995. Mechanisms of Emmetropization in the Aging Eye. Optometry and Vision Science. 72, 60-66.

Op de Beeck, H., Wagemans, J., Vogels, R., 2001. Inferotemporal neurons represent lowdimensional configurations of parameterized shapes. Nat Neurosci. 4, 1244-52.

Orban, G.A., Van Essen, D., Vanduffel, W., 2004. Comparative mapping of higher visual areas in monkeys and humans. Trends Cogn Sci. 8, 315-24. 
Parks, E.L., Madden, D.J., 2013. Brain connectivity and visual attention. Brain Connect. 3, 317-38.

Penfield, W., 1950a. The supplementary motor area in the cerebral cortex of man. Arch Psychiatr Nervenkr Z Gesamte Neurol Psychiatr. 185, 670-4.

Penfield, W., 1950b. Observations on the anatomy of memory. Folia Psychiatr Neurol Neurochir Neerl. 53, 349-51.

Penfield, W., 1957. Some observations on the functional organization of the human brain. Ideggyogy Sz. 10, 138-41.

Penfield, W., 1958. Some mechanisms of consciousness discovered during electrical stimulation of the brain. Proc Natl Acad Sci U S A. 44, 51-66.

Penny, W.D., et al., 2004. Comparing dynamic causal models. Neuroimage. 22, 1157-72.

Peters, A., Moss, M.B., Sethares, C., 2000. Effects of aging on myelinated nerve fibers in monkey primary visual cortex. J Comp Neurol. 419, 364-76.

Peters, A., Sethares, C., 2002. Aging and the myelinated fibers in prefrontal cortex and corpus callosum of the monkey. Journal of Comparative Neurology. 442, 277-291.

Rasmussen, T., Penfield, W., 1947. The human sensorimotor cortex as studied by electrical stimulation. Fed Proc. 6, 184.

Rasmussen, T., Penfield, W., 1948. Movement of head and eyes from stimulation of human frontal cortex. Res Publ Assoc Res Nerv Ment Dis. 27 (1 vol.), 346-61.

Riis, J.L., et al., 2008. Compensatory neural activity distinguishes different patterns of normal cognitive aging. Neuroimage. 39, 441-54.

Rolls, E.T., Tovee, M.J., 1995. Sparseness of the neuronal representation of stimuli in the primate temporal visual cortex. J Neurophysiol. 73, 713-26.

Roudaia, E., Bennett, P.J., Sekuler, A.B., 2008. The effect of aging on contour integration. Vision Res. 48, 2767-74.

Roudaia, E., et al., 2010. Spatiotemporal properties of apparent motion perception and aging. J Vis. 10, 5.

Sagi, Y., et al., 2012. Learning in the fast lane: new insights into neuroplasticity. Neuron. 73, 1195-203.

Salthouse, T.A., 1996. The processing-speed theory of adult age differences in cognition. Psychol Rev. 103, 403-28.

Salzman, C.D., Newsome, W.T., 1994. Neural mechanisms for forming a perceptual decision. Science. 264, 231-7.

Sampaio-Baptista, C., et al., 2013. Motor skill learning induces changes in white matter microstructure and myelination. J Neurosci. 33, 19499-503.

Sary, G., Vogels, R., Orban, G.A., 1993. Cue-invariant shape selectivity of macaque inferior temporal neurons. Science. 260, 995-7.

Sary, G., et al., 1995. Responses of monkey inferior temporal neurons to luminance-, motion-, and texture-defined gratings. J Neurophysiol. 73, 1341-54.

Sary, G., et al., 2006. Task-related modulation in the monkey inferotemporal cortex. Brain Res. 1121, 76-82.

Sáry, G., et al., 2004. Relationship between stimulus complexity and neuronal activity in the inferotemporal cortex of the macaque monkey. Cognitive Brain Research. 22, $1-12$.

Saygin, Z.M., et al., 2012. Anatomical connectivity patterns predict face selectivity in the fusiform gyrus. Nat Neurosci. 15, 321-7.

Schmolesky, M.T., et al., 1998. Signal timing across the macaque visual system. J Neurophysiol. 79, 3272-8.

Schmolesky, M.T., et al., 2000. Degradation of stimulus selectivity of visual cortical cells in senescent rhesus monkeys. Nat Neurosci. 3, 384-90. 
Scholz, J., et al., 2009. Training induces changes in white-matter architecture. Nat Neurosci. 12, 1370-1.

Sekuler, R., Hutman, L.P., Owsley, C.J., 1980. Human aging and spatial vision. Science. 209, 1255-6.

Shams, L., et al., 2001. Sound alters visual evoked potentials in humans. Neuroreport. 12, 3849-52.

Shams, L., Kim, R., 2010. Crossmodal influences on visual perception. Phys Life Rev. 7, 269-84.

Sheinberg, D.L., Logothetis, N.K., 1997. The role of temporal cortical areas in perceptual organization. Proc Natl Acad Sci U S A. 94, 3408-13.

Siegel, R.M., Andersen, R.A., 1988. Perception of three-dimensional structure from motion in monkey and man. Nature. 331, 259-61.

Sincich, L.C., et al., 2004. Bypassing V1: a direct geniculate input to area MT. Nat Neurosci. 7, 1123-8.

Sivyer, B., et al., 2010. Synaptic inputs and timing underlying the velocity tuning of direction-selective ganglion cells in rabbit retina. J Physiol. 588, 3243-53.

Skottun, B.C., 2015. On the use of spatial frequency to isolate contributions from the magnocellular and parvocellular systems and the dorsal and ventral cortical streams. Neuroscience \& Biobehavioral Reviews. 56, 266-275.

Smith, S.M., et al., 2004. Advances in functional and structural MR image analysis and implementation as FSL. Neuroimage. 23 Suppl 1, S208-19.

Smith, S.M., et al., 2007. Acquisition and voxelwise analysis of multi-subject diffusion data with tract-based spatial statistics. Nat Protoc. 2, 499-503.

Smith, S.M., Nichols, T.E., 2009. Threshold-free cluster enhancement: addressing problems of smoothing, threshold dependence and localisation in cluster inference. Neuroimage. 44, 83-98.

Spear, P.D., 1993. Neural bases of visual deficits during aging. Vision Res. 33, 2589-609.

Spear, P.D., et al., 1994. Effects of aging on the primate visual system: spatial and temporal processing by lateral geniculate neurons in young adult and old rhesus monkeys. J Neurophysiol. 72, 402-20.

Staempfli, P., et al., 2008. Combining fMRI and DTI: a framework for exploring the limits of fMRI-guided DTI fiber tracking and for verifying DTI-based fiber tractography results. Neuroimage. 39, 119-26.

Stanley, G.B., et al., 2012. Visual orientation and directional selectivity through thalamic synchrony. J Neurosci. 32, 9073-88.

Stepan-Buksakowska, I., et al., 2014. Cortical and subcortical atrophy in Alzheimer disease: parallel atrophy of thalamus and hippocampus. Alzheimer Dis Assoc Disord. 28, 65-72.

Sutton, S., et al., 1965. Evoked-potential correlates of stimulus uncertainty. Science. 150, 1187-8.

Tamura, H., Tanaka, K., 2001. Visual response properties of cells in the ventral and dorsal parts of the macaque inferotemporal cortex. Cereb Cortex. 11, 384-99.

Tanaka, K., et al., 1991. Coding visual images of objects in the inferotemporal cortex of the macaque monkey. J Neurophysiol. 66, 170-89.

Tanaka, K., 1997. Mechanisms of visual object recognition: monkey and human studies. Curr Opin Neurobiol. 7, 523-9.

Teder-Salejarvi, W.A., et al., 2002. An analysis of audio-visual crossmodal integration by means of event-related potential (ERP) recordings. Brain Res Cogn Brain Res. 14, 106-14. 
Thiebaut de Schotten, M., et al., 2011. A lateralized brain network for visuospatial attention. Nat Neurosci. 14, 1245-6.

Thompson, K.G., et al., 1996. Perceptual and motor processing stages identified in the activity of macaque frontal eye field neurons during visual search. J Neurophysiol. 76, 4040-55.

Thompson, R.F., Patterson, M.M., 2013. Bioelectric Recording Techniques: Electroencephalography and Human Brain Potentials, Vol., Elsevier Science.

Tigges, J., et al., 1988. Survival Rate and Life-Span of Rhesus-Monkeys at the YerkesRegional-Primate-Research-Center. American Journal of Primatology. 15, 263 273.

Tomassini, V., et al., 2011. Structural and Functional Bases for Individual Differences in Motor Learning. Human Brain Mapping. 32, 494-508.

Tompa, T., Sary, G., 2010. A review on the inferior temporal cortex of the macaque. Brain Res Rev. 62, 165-82.

Toosy, A.T., et al., 2004. Characterizing function-structure relationships in the human visual system with functional MRI and diffusion tensor imaging. Neuroimage. 21, 1452-63.

Tootell, R.B., et al., 1995. Visual motion aftereffect in human cortical area MT revealed by functional magnetic resonance imaging. Nature. 375, 139-41.

Tremblay, M.E., et al., 2012. Effects of aging and sensory loss on glial cells in mouse visual and auditory cortices. Glia. 60, 541-58.

Treue, S., Maunsell, J.H., 1996. Attentional modulation of visual motion processing in cortical areas MT and MST. Nature. 382, 539-41.

Turkington, T., 2011. PET Imaging Basics. In: Clinical PET-CT in Radiology. Vol., P. Shreve, D.W. Townsend, ed.^eds. Springer New York, pp. 21-28.

Ungerleider, L.G., Haxby, J.V., 1994. 'What' and 'where' in the human brain. Curr Opin Neurobiol. 4, 157-65.

Ungerleider, L.G., Mishkin, M.,, 1982. Two cortical visual systems. In: Analysis of Visual Behavior. Vol., D. Ingle, M.A. Goodale, R.J.W. Mansfield, ed.^eds. MIT Press.

van den Heuvel, M., et al., 2008. Microstructural organization of the cingulum tract and the level of default mode functional connectivity. J Neurosci. 28, 10844-51.

Van der Burg, E., et al., 2008. Pip and pop: nonspatial auditory signals improve spatial visual search. J Exp Psychol Hum Percept Perform. 34, 1053-65.

Van Impe, A., et al., 2012. White matter fractional anisotropy predicts balance performance in older adults. Neurobiol Aging. 33, 1900-12.

Vroomen, J., Keetels, M., 2010. Perception of intersensory synchrony: a tutorial review. Atten Percept Psychophys. 72, 871-84.

Walter, W.G., et al., 1964. Contingent Negative Variation: An Electric Sign of Sensorimotor Association and Expectancy in the Human Brain. Nature. 203, 3804.

Wang, H., et al., 2006. Functional degradation of visual cortical cells in aged rats. Brain Res. 1122, 93-8.

Wang, Y., et al., 2002. Contribution of GABAergic inhibition to receptive field structures of monkey inferior temporal neurons. Cereb Cortex. 12, 62-74.

Wang, Y.C., et al., 2005. Degradation of signal timing in cortical areas V1 and V2 of senescent monkeys. Cerebral Cortex. 15, 403-408.

Wang, Y.Z., 2001. Effects of aging on shape discrimination. Optom Vis Sci. 78, 447-54.

Watkins, S., et al., 2006. Sound alters activity in human V1 in association with illusory visual perception. Neuroimage. 31, 1247-56. 
Watkins, S., et al., 2007. Activity in human V1 follows multisensory perception. Neuroimage. 37, 572-578.

Watson, A.B., Pelli, D.G., 1983. QUEST: a Bayesian adaptive psychometric method. Percept Psychophys. 33, 113-20.

Wedeen, V.J., et al., 2005. Mapping complex tissue architecture with diffusion spectrum magnetic resonance imaging. Magn Reson Med. 54, 1377-86.

Weymouth, A.E., McKendrick, A.M., 2012. Shape perception is altered by normal aging. Invest Ophthalmol Vis Sci. 53, 3226-33.

Wiesel, T.N., Hubel, D.H., 1963. Single-cell responses in striate cortex of kittens deprived of vision in one eye. J Neurophysiol. 26, 1003-17.

Wilson, J.T., 1987. Interaction of simultaneous visual events. Perception. 16, 375-83.

Winkler, A.M., et al., 2014. Permutation inference for the general linear model. Neuroimage. 92, 381-97.

Xi, M.C., et al., 1999. Changes in the axonal conduction velocity of pyramidal tract neurons in the aged cat. Neuroscience. 92, 219-25.

Yang, Y., et al., 2008. Aging affects contrast response functions and adaptation of middle temporal visual area neurons in rhesus monkeys. Neuroscience. 156, 748-57.

Yang, Y., et al., 2009a. Aging affects response variability of V1 and MT neurons in rhesus monkeys. Brain Res. 1274, 21-7.

Yang, Y., et al., 2009b. Aging affects the neural representation of speed in Macaque area MT. Cereb.Cortex. 19, 1957-1967.

Yin, X., et al., 2012. Anatomical substrates of the alerting, orienting and executive control components of attention: focus on the posterior parietal lobe. PLoS One. 7, e50590.

Yu, S., et al., 2006. Functional degradation of extrastriate visual cortex in senescent rhesus monkeys. Neuroscience. 140, 1023-9.

Zatorre, R.J., Fields, R.D., Johansen-Berg, H., 2012. Plasticity in gray and white: neuroimaging changes in brain structure during learning. Nat Neurosci. 15, 52836.

Zeki, S., et al., 1991. A direct demonstration of functional specialization in human visual cortex. J Neurosci. 11, 641-9.

Zihl, J., von Cramon, D., Mai, N., 1983. Selective disturbance of movement vision after bilateral brain damage. Brain. 106 (Pt 2), 313-40. 
$\underline{\text { Appendix }}$ 
I. 
Research Report

\section{Audio-visual integration through the parallel visual pathways}

Péter Kaposuári ${ }^{1, a}$, Gergö Csete $^{a, b, 1}$, Anna Bognár ${ }^{a}$, Péter Csibri ${ }^{a}$, Eszter Tóth ${ }^{b}$, Nikoletta Szabób,c, László Vécsei ${ }^{b, d}$, Gyula Sáry ${ }^{a, *}$, Zsigmond Tamás Kincses ${ }^{b, c}$

aDepartment of Physiology, University of Szeged, Szeged, Hungary

bDepartment of Neurology, Albert Szent-Györgyi Clinical Centre, University of Szeged, Szeged, Hungary

'International Clinical Research Centre, St. Anne's University Hospital Brno, Brno, Czech Republic

${ }^{\mathrm{d}}$ MTA-SZTE Neuroscience Research Group, University of Szeged, Hungary

A R T I C L E I N F O

Article history:

Accepted 23 June 2015

Available online 9 July 2015

\section{Keywords:}

MRI

DTI

TBSS

Multisensory

Doubleflash

\begin{abstract}
A B S T R A C T
Audio-visual integration has been shown to be present in a wide range of different conditions, some of which are processed through the dorsal, and others through the ventral visual pathway. Whereas neuroimaging studies have revealed integration-related activity in the brain, there has been no imaging study of the possible role of segregated visual streams in audio-visual integration.

We set out to determine how the different visual pathways participate in this communication. We investigated how audio-visual integration can be supported through the dorsal and ventral visual pathways during the double flash illusion. Low-contrast and chromatic isoluminant stimuli were used to drive preferably the dorsal and ventral pathways, respectively. In order to identify the anatomical substrates of the audio-visual interaction in the two conditions, the psychophysical results were correlated with the white matter integrity as measured by diffusion tensor imaging.The psychophysiological data revealed a robust double flash illusion in both conditions. A correlation between the psychophysical results and local fractional anisotropy was found in the occipito-parietal white matter in the low-contrast condition, while a similar correlation was found in the infero-temporal white matter in the chromatic isoluminant condition. Our results indicate that both of the parallel visual pathways may play a role in the audio-visual interaction. c 2015 Published by Elsevier B.V.
\end{abstract}

1.

\section{Introduction}

One brief flash and two short tones presented simultaneously induce the illusion of a second flash, called the double flash

*Corresponding author.

E-mail address: sary.gyula@med.u-szeged.hu (G. Sáry).

${ }^{1}$ These authors contributed equally to the experiments.

http://dx.doi.org/10.1016/j.brainres.2015.06.036 0006-8993/O 2015 Published by Elsevier B.V. illusion. Since the first description of the phenomenon (Wilson, 1987), there have been several studies looking for the possible background mechanisms. Event-related potential studies demonstrated that auditory stimuli could 
influence the activity of the visual cortex (Shams et al., 2001; Teder-Salejarvi et al., 2002) and an illusory flash-related gamma burst was found in primary visual areas (Bhattacharya et al., 2002; Mishra et al., 2007). Functional MRI investigations revealed illusory flash-related brain activity in the superior colliculus, the primary visual cortex and the right superior temporal sulcus (STS) (Watkins et al., 2006). These findings suggest that such interaction of the bimodal information could be based on the communication between the primary visual cortex, the STS and the primary auditory cortex (Mishra et al., 2007; Watkins et al., 2006, 2007).

The higher visual cortical areas from the primary visual cortex towards the associative areas in primates are arranged to form two major information-processing streams, the dorsal and ventral pathways. These streams are traditionally regarded as the systems responsible for the representation of the 'where' and the 'what', respectively, in the visual world (Haxby et al., 1991; Ungerleider and Haxby, 1994; Wilson, 1987). The dorsal stream originates from the magnocellular pathway and is principally concerned with movement, speed of motion and location in space. The ventral stream originates from the parvocellular pathway and is responsible for the exact identification of what is seen, such as fine structure and colour These pathways can easily be distinguished by modulating the contrast of the stimuli: stimuli with high contrast and colour are processed predominantly in the ventral stream, while stimuli with low contrast are processed in the dorsal stream (Brannan and Bodis-Wollner, 1991; Legge, 1978).

Earlier studies suggested that the double flash illusion is primarily mediated by the dorsal pathway. However, it has not yet been investigated whether the phenomenon can be evoked over both visual streams, and no systematic investigation of the involved neural structures of the dorsal and ventral streams in the illusion has yet been performed.

Electrophysiological and functional imaging studies offer a unique opportunity to investigate the contributions of various brain regions to certain functions. It is also known that function is deeply rooted in the anatomical structure, and behavioural performance is strongly influenced by the properties of the underlying brain structures. It was shown that correlation between the individual structural variability and behavioural performance can identify the neuroanatomical structures involved (Maguire et al., 2000). Furthermore, the microstructural integrity of the white matter as defined by diffusion tensor imaging is capable of revealing the coupling of structure and function (Csete et al., 2014; Johansen-Berg, 2010).

In this study we investigated the contributions of the parallel visual pathways to the double flash illusion, and aimed to identify the structural background of this processing. Using auditory stimuli to trigger the phenomenon, we set out to induce the illusion with (i) low-contrast stimuli to drive the dorsal pathway, which is sensitive to contrast and insensitive to colour information and, (ii) isoluminant stimuli to drive the ventral pathway, which is less sensitive to contrast differences. Although the distinction is not complete, this approach might be useful to investigate how additional auditory information can influence the different parts of the visual system (Kaposvari et al., 2014). We hypothesised that a better performance of an individual might relate to the higher structural integrity of the pathways involved in the processing of the stimulus. Hence, the behavioural performance in the two conditions was correlated with the diffusion MRI measured white matter microstructural integrity in order to reveal the structural background of the double flash illusion.

\section{Results}

\subsection{Behavioural data}

The subjects were tested in a double-flash paradigm, in which one or two visual stimuli following each other were presented together with a single or two consecutive tones. The measurements were repeated in two conditions: when the visual stimuli were isoluminant compared to the background (IL) (putative ventral pathway processing) and a low-contrast (LC) condition (presumably processed through the dorsal steam). According to the Signal Detection Theory, a perceptual sensitivity $\left(d^{\prime}\right)$ was calculated from the correct identification of the second flashes ('hit') and from the 'false alarms'.

The paired t-test between $d$ ' values in control (one flash and two flashes without tones) and double-flash (one flash with two tones and two flashes and two tones) subconditions indicated the existence of the double flash illusion in both the LC condition $(p<0.0003)$ and the IL condition $(p<0.0027)$.The criterion revealed significant $(p<0.01)$ negative bias for the double flash as compared with the control criterion in both conditions (Table 1). This shows that two tones biased the participants to report two flashes instead of one for doubleflash illusions, but not the general response bias.

\subsection{Correlation of behavioural data with white matter} microstructural integration

In order to reveal small inter-individual variations in the white matter microstructure, which might explain the subject-to-subject differences in perceptual sensitivity we correlated the behavioural data with the diffusion parameters of the white matter.

Table 1 - The mean and standard error (SEM) of the $d^{\prime}$ and criterion values in the behavioural tests. Please note that sin the values of "Hit" and "False alarm" were rounded, the $d$ ' values in the table above might deviate from the correct mathematical value.

\begin{tabular}{llllllllll} 
Conditions & & $d^{\prime}$ mean & \pm SEM & C mean & \pm SEM & Hit mean & \pm SEM & False alarm mean & \pm SEM \\
\hline \multirow{2}{*}{ Isoluminant } & control & 3.592 & 0.211 & 0.087 & 0.140 & 0.9084 & 0.0229 & 0.0813 & 0.0253 \\
& double flash & 1.721 & 0.244 & -1.605 & 0.180 & 0.9750 & 0.0108 & 0.6667 & 0.0761 \\
\multirow{2}{*}{ Low contrast } & control & 3.461 & 0.216 & -0.057 & 0.130 & 0.9229 & 0.0210 & 0.0917 & 0.0244 \\
& double flash & 1.917 & 0.270 & -1.604 & 0.166 & 0.9729 & 0.0115 & 0.6521 & 0.0811 \\
\hline
\end{tabular}




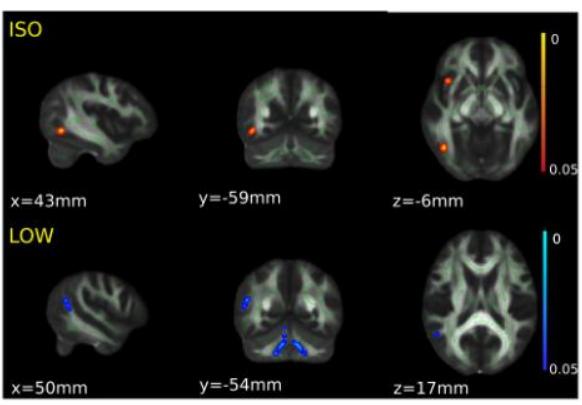

Fig. 1 - Correlation of FA with a higher likelihood of perceiving a double-flash illusion. White matter microstructure as measured by FA, showed correlation with the perceptual sensitivity to double flash illusion in the isoluminant (upper row) and low-contrast condition (lower row). The identified white matter regions overlap with the ventral and dorsal visual pathways respectively. Images are overlaid on the FMRIB58_FA standard image. The mean FA skeleton, thresholded at 0.2 , is depicted in green shades. Significant clusters are indicated in red for the isoluminant and in blue for the low-contrast condition. A thickened version of the significant cluster is used to facilitate visualisation. (For interpretation of the references to colour in this figure legend, the reader is referred to the web version of this article.)

In the IL condition, a significant positive correlation of the behavioural data (the likelihood of perceiving a double-flash illusion) and the fractional anisotropy (FA) was found in the juxtacortical infero-temporal white matter on the right and in the bilateral insulae by the Tract-Based Spatial Statistic (TBSS) analysis (Fig. 1 and Table 2).

In the LC condition, a significant positive correlation was observed between FA and the likelihood of perceiving a double-flash illusion in the juxtacortical white matter of the parieto-occipital junction on the right side, the right frontal white matter under the superior frontal gyrus and bilaterally in the deep cerebellar white matter (Fig. 1 and Table 3).

TBSS analysis revealed no significant correlation with any other diffusion parameters (MD, AD and $\mathrm{RD}$ ).

\subsection{Connectivity analysis}

The probabilistic tractography from the infero-temporal white matter region, which revealed a high correlation with the likelihood of perceiving a double-flash illusion in the IL condition, showed tracks running along the inferior border of the temporal lobe through the inferior fronto-occipital fascicle (an association pathway connecting the occipital lobe with the frontal lobe) and the inferior longitudinal fascicle (an association pathway connecting the occipital lobe to the temporal lobe) (Fig. 2)

In the LC condition, the tractography initiated from the juxtacortical parieto-occipital cluster of the TBSS analysis showed fibres along the putative arcuate fascicle, running towards the frontal lobe (Fig. 2).

\section{Discussion}

In this study, we evoked a double-flash illusion through the dorsal and ventral streams by using low contrast and chromatic-isoluminant stimuli. Moreover, we identified correlations between the microstructure of various white matter tracks and likelihood of perceiving double-flash illusion. This correlation was found along the putative ventral and dorsal visual streams in the two conditions in which stimuli were predominantly driving the 'what' and 'where' pathways.

The visual system is traditionally subdivided to two parallel information processing pathways. The dorsal stream processes temporal information better, but its spatial resolution is inferior to that of the ventral stream, runs toward the parietal lobe. Contrary, the ventral stream, which is important for object and colour recognition, is running toward the infero-temporal structures. Importantly, in experimental protocols the two pathways can be separated by presenting low and high contrast stimuly, respectively. Finding correlation between the likelihood of perceiving double-flash illusion and white matter integrity along the ventral and dorsal streams in the two conditions driving preferentially one or the other pathway, indicate that audio-visual integration may be processed in both systems depending on the stimulus parameters.

Evidence was reported earlier of audio-visual integration occurring through the ventral stream. In a metacontrast masking paradigm, Jaekl and Harris who were able to eliminate the inhibitory effect of M-pathway-related transient activity on the P-pathway by applying a concurrent auditory stimulus, expected to increase the activity within the Ppathway (Jaekl and Harris, 2009). In the same paper, they provided further evidence by applying an orientation discrimination paradigm with an additional auditory stimulus. Here, the orientation threshold was also modulated by the concurrent auditory stimulus, which they considered to be an indication that audio-visual interactions can occur through the ventral pathway (Jaekl and Harris, 2009). Furthermore, by using chromatic stimuli, Leo et al. (2008) were able to show that, in the case of short-wavelength stimuli, processed by the parvocellular system, a significant reaction time advance can be produced by a concurrent auditory stimulus.

In contrast, there is also evidence that audio-visual integration may occur through the dorsal pathway. Jaek and Soto-Faraco (2010) showed that auditory stimuli had significant effects on the contrast sensitivity in a steady, but not in a pulsed-pedestal condition, the former predominantly testing the dorsal, and the latter the ventral pathway. Furthermore, an audio-visual interaction was represented at lower, but not at higher spatial frequencies, which favours the role of the M-pathway (Jaekl and Soto-Faraco, 2010). On the use of stimuli, which probably preferentially drives the Mpathway, fMRI activation differences were also detected along the M-pathway (Watkins et al., 2006, 2007).

The results listed above are seemingly contradictory, though our results relating to a double-flash illusion with low contrast and with chromatic-isoluminant stimuli suggest that audio-visual integration can be evoked through both the dorsal and the ventral streams. 


$\begin{aligned} & \text { Table 2 - Anatomical localisation of correlations of FA with the likelihood of perceiving a double-flash illusion in the } \\
& \text { isoluminant condition. Coordinates represent standard space coordinates in the FMRIB58_FA template. }\end{aligned}$
\begin{tabular}{lllllrl} 
& $p<$ & No. of voxels & $X(\mathrm{~mm})$ & $\mathrm{Y}(\mathrm{mm})$ & $\mathrm{Z}(\mathrm{mm})$ \\
\hline Right insular WM & 0.001 & 56 & 36 & 12 & -14 \\
Right infero-temporal WM & 0.001 & 33 & 45 & -60 & -8 \\
Left insular WM & 0.001 & 10 & -32 & 21 & 0 \\
\hline
\end{tabular}

It is important to note that no stimulus is processed exclusively through the ventral or the dorsal pathway; the stimuli in our investigation were designed to drive preferentially the magno- or the parvocellular pathways (Kaposvan et al., 2014).

Further support of audio-visual integration taking place through both the ventral and dorsal pathways comes from the results of the DTI analysis. The likelihood of the perception of illusion in the low-contrast condition correlated with the white matter integrity of the parieto-occipital junction and the frontal white matter. Not only is the parieto-occipital region in the close vicinity of the dorsal pathway cortical regions, but its connectivity pattern resembles the dorsal pathway (Haxby et al., 1991; Ungerleider and Haxby, 1994).

Similarly, the correlation of behavioural measures with tissue integrity in the infero-temporal white matter in the case of the chromatic-isoluminant condition strongly supports the possible involvement of the ventral pathway in the audio-visual integration.

Interestingly, the correlations between the probability of double-flash illusion and the white matter integrity were lateralised, however it has to be noted that there were correlations found on the other side also, but they were just below the threshold. Nevertheless, having lateralised result are not surprising: several studies showed asymmetric function and structure in the visual system (Antal et al., 2004 Dreessen de Gervai et al., 2014).

We also found in the literature significant correlation between structure and function in non-visual pathways. Electrophysiology and fMRI investigation showed that the insula is participating in multimodal integration (Calvert et al., 2001) and recently it was demonstrated that isochronous timing in an audiovisual task has a positive influence on insular activity (Marchant and Driver, 2013). Also several researches pointed out the importance of cerebellum in audio-visual integration (Pfordresher et al., 2014; Proverbio et al., 2014).

White matter integrity and structural connectivity were recently of interest as concerns the determination of the connection between structure and function. The variation of the connectivity profile of certain regions can predict a variation in function (Behrens et al., 2006; Johansen-Berg et al., 2004; Klein et al., 2007; Saygin et al., 2012). Local diffusion features of the white matter supported to be related to the behavioural performance (Csete et al., 2014; JohansenBerg, 2010). A higher FA in the corpus callosum was related to a better bimanual coordination (Johansen-Berg et al., 2007). A training-induced FA increase has been detected in the intraparietal sulcus (Scholz et al., 2009). It has also been shown that a greater BOLD response in the visual cortex correlates with a higher FA in the optic radiation (Toosy et al., 2004). Neuroimaging and histological studies have been combined recently that demonstrated that spatial learning and motor learning result in locally enhanced myelination (BlumenfeldKatzir et al., 2011; Sampaio-Baptista et al., 2013), which is reflected in enhanced FA in the diffusion MRI.

The relationships between these white matter diffusion properties and histological properties are not yet clear, but nerve diameter and myelination that relate to the microstructure measurable with DTI can also relate to the conduction velocity and dispersion of the neuronal signal (Hodgkin and Huxley, 1952). As regarding the functional significance of the diffusion-measured microstructure, recently it was shown that the FA of the white matter pathway neurophysiology measured functional connectivity of the connected regions is correlated (Boorman et al., 2007; Neubert et al., 2010).

\section{Experimental procedures}

\subsection{Subjects}

Sixteen healthy subjects ( 10 females; mean age of 27.4 years) were enroled in the study. None of them suffered from any neurological or psychiatric diseases. All of them had normal or corrected-to-normal (20/20) visual acuity and good colour vision. The study was approved by the ethical committee of the University of Szeged (Ref. no.: 87/2009) and all study participants gave their written informed consent in accordance with the Declaration of Helsinki.

\subsection{Behavioural test}

The subjects sat in a sound-attenuated dark room with their heads resting on a chin and forehead support. Their eyes were $57 \mathrm{~cm}$ away from the screen (ViewSonic monitor, inch diameter, $800 \times 600,60.0 \mathrm{~Hz}$ ) and the speakers. The two computer speakers were positioned symmetrically, on each side of the monitor, at $25^{\circ}$ from the fixation point. The subjects had to fix their gaze on a black fixation dot at the middle of the monitor. A disc subtending a visual angle of $1.5^{\circ}$ was displayed in a peripheral position at 9.25 eccentricity below the fixation point, as a visual stimulus (Watkins et al., 2007)

Two conditions were investigated: an isoluminant (IL) condition, where a red disc was presented on a green background $\left(8.9 \mathrm{~cd} / \mathrm{m}^{2}\right)$, and a low-contrast (LC) condition, where we used a light-grey disc $\left(9.7 \mathrm{~cd} / \mathrm{m}^{2}\right.$, contrast $\left.9 \%\right)$ on a darker grey background $\left(8.9 \mathrm{~cd} / \mathrm{m}^{2}\right)$. The conditions were presented in a semi-random sequence.

The conditions contained 6 subconditions: 6 variations of flashes (one flash, one flash with one tone, one flash with two tones, two flashes, two flashes with one tone, and two flashes with two tones). One subcondition consisted of 40 repetitions 


\begin{tabular}{|c|c|c|c|c|c|}
\hline & $p<$ & No. of voxels & $\mathrm{X}(\mathrm{mm})$ & $\mathrm{Y}(\mathrm{mm})$ & $\mathrm{Z}(\mathrm{mm}$ \\
\hline Cerebellar WM & 0.001 & 582 & -17 & -65 & -33 \\
\hline Right parieto-occipital junction & 0.001 & 53 & 52 & -51 & 9 \\
\hline Right fronto-polar WM & 0.001 & 10 & 22 & 56 & 7 \\
\hline Right superior frontal WM & 0.005 & 4 & 17 & 21 & 51 \\
\hline
\end{tabular}

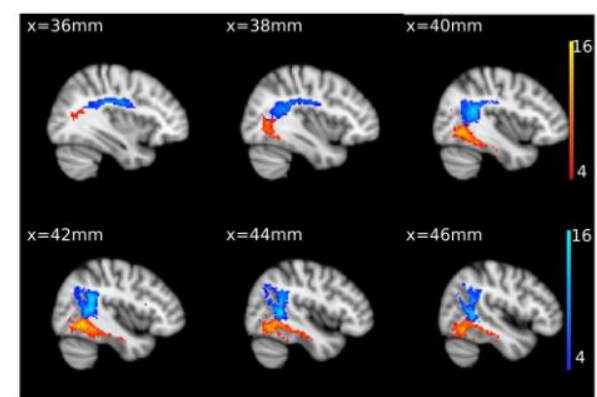

$85 \mathrm{~ms}$. The duration of the tones $(3.5 \mathrm{kHz}, 70 \mathrm{dBSPL})$ was $10 \mathrm{~ms}$ and was presented at the same time as the first flash. The SOA between the two tones was $85 \mathrm{~ms}$. The previously mentioned experiments used auditory and visual stimuli slightly shifted in time, but as reported, the two designs with simultaneously presented or shifted stimuli resulted in only slight differences (Watkins et al., 2006). After the presentations of the flashes and tones, the subject had to decide whether one or two discs were displayed, independently of the tones, and press the left (one flash) or right arrow (two flashes) button on the keyboard with the dominant hand. After the subject had pressed a key, the grey background $\left(8.9 \mathrm{~cd} / \mathrm{m}^{2}\right)$ appeared for the inter-trial interval of $1000 \mathrm{~ms}$. Feedback concerning the correctness or not of the responses was not provided.

\subsection{Analysis of behavioural data}

Signal detection theory was used to analyse the behavioural results. With this method, the sensitivity of the subjects towards the visual stimuli could be described. The aim was to verify that the illusions were caused by changes in perceptual sensitivity, but not by the general response bias. The sensitivity is expressed as

$d^{\prime}=z(H)-z(F)$,

Fig. 2 - Connectivity of clusters showing the correlation between FA and behavioural data in the isoluminant (red) and low-contrast (blue) conditions. The white matter fibres identified by the tractography correspond to the ventral and dorsal visual pathways in the isoluminant and low-contrast conditions respectively. The binary cluster masks were used as seed mask for each subject. Five thousand streamline samples from each seed voxel were drawn to build up a connectivity distribution that was thresholded for 1000 particles for each subject and binarized. Population connectivity maps were derived for controls by adding these masks together and thresholding at four (Pathways passing through the given voxel in at least four subjects). (For interpretation of the references to colour in this figure legend, the reader is referred to the web version of this article.)

of the trial, and thus one block contained 240 semi-randompresented trials.

The presentation of the trial started with the conditionspecific green background in the IL condition and with the grey background in the LC condition. After $200 \mathrm{~ms}$ one or two discs were presented for $17 \mathrm{~ms}$ on this background, without or with one or two tones, according to the given condition. The stimulus onset asynchrony (SOA) between two flashes was

where $d^{\prime}$ is the sensitivity, and $z$ is the inverse of the normal cumulative distribution. In one block, we calculated three $d$ ' values for two subconditions. The correct identification of the second flash was accepted as a 'hit' $(\mathrm{H})$; if the subject reported one flash instead of two, it was recorded as a 'miss'. When one flash was reported as two, we accepted it as a 'false alarm' ( $F$ ) and the correct identification of one flash was accepted as a 'correct rejection'. To calculate the $d$ ' value for the control, we used the two subconditions without tones (one flash and two flashes). For double flashes, we used the two subconditions with two tones (one flash with two tones and two flashes with two tones). To examine the power of the illusions, we compared the control $d$ ' value with that for fusion or the double flash by the paired t-test (Watkins et al., 2006). A criterion (C) was calculated to indicate the response bias via the expression (MacMillan and Creelman, 2005):

$$
C=-[z(\mathrm{pH})+z(\mathrm{pF})] / 2,
$$

A positive value of $C$ indicates the bias when the subjects rather report one flash, while a negative value indicates when they rather report two flashes. 


\subsection{Data acquisition}

Neuroimaging data acquisitions were carried out on a $1.5 T$ GE Signa Excite HDxt MR Scanner (GE Healthcare, Chalfont St. Giles, UK). Three-dimensional spoiled gradient echo images (FSPGR: echo time [TE]: $4.1 \mathrm{~ms}$; repetition time [TR]: $10.276 \mathrm{~ms}$ matrix: $256^{*} 256$; field of view [FOV]: $25 \mathrm{~cm}^{*} 25 \mathrm{~cm}$; flip angel: $15^{\circ}$ in-plane resolution: $1^{*} 1 \mathrm{~mm}^{2}$; slice thickness: $1 \mathrm{~mm}$ ) and 60 directions diffusion-weighted images with 6 non-diffusionweighted reference volume (TE: $93.8 \mathrm{~ms}$; TR: 16,000 ms; matrix 96*96; FOV: $23^{*} 23 \mathrm{~cm}^{2}$; flip angle: 90 ; in-plane resolution: $2.4^{*} 2.4 \mathrm{~mm}^{2}$; slice thickness: $2.4 \mathrm{~mm}$; b: $1000 \mathrm{~s} / \mathrm{m} 2$; number of excitations [NEX]: 2; array spatial sensitivity encoding technique [ASSET]) were acquired for all subjects.

\subsection{Data analysis}

Diffusion data were corrected for eddy currents and movements artefacts by 12 degrees of freedom affine linear registration to the first non-diffusion-weighted reference image. Diffusion tensors at each voxel were fitted by an algorithm included in FMRIB's Diffusion Toolbox (FDT) of FSL (v.4.0) (Smith et al., 2004). Fractional anisotropy (FA), mean (MD), axial (AD) and radial diffusivity $(\mathrm{RD})$ was computed for the whole brain. In order to reduce the possible errors arising from misalignment of the images, we used the Tract Based Spatial Statistics (TBSS) method (Smith et al., 2007). For all subjects, the FA images were aligned into a common space, using the non-linear registration tool, FNIRT, which uses a b-spline representation of the registration warp field. The mean FA image was created and then thresholded at $\mathrm{FA}=0.2$, deriving a mean FA skeleton representing the centres of all tracts common to the group. The aligned FA data for each subjects were then projected onto this skeleton and the resulting data were fed into voxel-wise cross-subject statistics. Modelling and inference with the stan dard general linear model design set-up was accomplished by using permutation-based cluster analysis $(n=5000)$ as implemented in the FSL software package (Nichols and Holmes, 2002). The design encoded for the differences of the $d$ ' value of the double-flash and the control $d$ ' in two condition (isoluminant and low-contrast) from psychophysical measurements. Statistical images were thresholded by threshold free cluster enhancement (Smith and Nichols, 2009). Since our task was a relatively low level perceptual task, it would have been over-conservative to correct for multiple correlations based on the total number of voxels. Hence, we used the non-corrected stats thresholded at a $1 \%$ significance level. Only clusters larger than 5 voxels were considered for discussion. The same analysis for $\mathrm{MD}, \mathrm{AD}$ and $\mathrm{RD}$ was repeated by projecting the various diffusion parameters to the FA skeleton.

The connectivity of the regions, which showed significan correlation with the behavioural data, was defined by the probabilistic tractography (FDT, part of FSL: www.fmrib.ox.ac. $\mathrm{uk} / \mathrm{fs} / / \mathrm{fdt})$. A multifibre diffusion model was fitted that estimates the probability distribution in the direction of 1 or more fibre populations at each voxel (Behrens et al., 2007). Probabilistic tractography was then performed on any brain voxel by tracing streamline samples through these probabilistic distributions in the fibre direction. For tractography, we generated 5000 streamline samples from each seed voxel to build up a connectivity distribution. The number of these samples passing through each brain voxel is interpreted as proportional to the probability of the connection to the seed voxel. By fitting a multifibre model to our diffusion data, we were able to trace pathways through regions of fibre crossing (Behrens et al., 2007). The seed masks were binary cluster-masks of the TBSS analysis.

\section{Acknowledgments}

This study was supported by the Neuroscience Research Group of the Hungarian Academy of Sciences and University of Szeged, project FNUSA-ICRC (no. CZ.1.05/1.1.00/02.0123) from the European Regional Development Fund, the National Brain Research Program (Grant no. KTIA_13_NAP-A-II/20.)and the Országos Tudományos Kutatási Alapprogramok (OTKA) [PD 104715] grant. The publication is supported by the European Union and co-funded by the European Social Fund. (Project title: "Telemedicine-focused research activities in the field of Mathematics, Informatics and Medical sciences" Project number: TÁMOP-4.2.2.A-11/1/KONV-2012-0073). This research was supported by the European Union and the State of Hungary, cofinanced by the European Social Found in the framework of TÁMOP 4.2.4.A/2-11-1-2012-0001 'National Excellence Program'. A. Bognár, P. Csibri and Gy. Sáry were supported by Országos Tudományos Kutatási Alapprogramok (OTKA) K83671. MR imaging was carried out by Euromedic Diagnostic Ltd., Szeged.

REFERENCES

Antal, A., Nitsche, M.A., Kincses, T.Z., Lampe, C., Paulus, W., 2004. No correlation between moving phosphene and motor thresholds: a transcranial magnetic stimulation study. Neuroreport 15, 297-302.

Behrens, T.E., Jenkinson, M., Robson, M.D., Smith, S.M., JohansenBerg, H., 2006. A consistent relationship between local white matter architecture and functional specialisation in medial frontal cortex. Neuroimage 30, 220-227,

Behrens, T.E., Berg, H.J., Jbabdi, S., Rushworth, M.F., Woolrich, M. W., 2007. Probabilistic diffusion tractography with multiple fibre orientations: what can we gain? Neuroimage 34 . 144-155.

Bhattacharya, J., Shams, L., Shimojo, S., 2002. Sound-induced illusory flash perception: role of gamma band responses. Neuroreport 13, 1727-1730.

Blumenfeld-Katzir, T., Pasternak, O., Dagan, M., Assaf, Y., 2011. Diffusion MRI of structural brain plasticity induced by a learning and memory task. PLoS One 6, e20678.

Boorman, E.D., O'Shea, J., Sebastian, C., Rushworth, M.F., Johansen-Berg, H., 2007. Individual differences in whiteJohansen-Berg, H., 2007. Individual differences in white matter microstructure reflect variation in functional

Brannan, J.R., Bodis-Wollner, L., 1991. Evidence for two systems mediating perceived contrast. Vis. Neurosci. 6, 587-592.

Calvert, G.A., Hansen, P.C., Iversen, S.D., Brammer, M.J., 2001 Detection of audio-visual integration sites in humans by application of electrophysiological criteria to the BOLD effect. Neuroimage 14, 427-438.

Csete, G., Szabo, N., Rokszin, A., Toth, E., Braunitzer, G., Benedek, G., Vecsei, L., Kincses, Z.T., 2014. An investigation of the white matter microstructure in motion detection using diffusion MRI. Brain Res. 1570, 35-42. 
Dreessen de Gervai, P., Sboto-Frankenstein, U.N., Bolster, R.B. Thind, S., Gruwel, M.L., Smith, S.D., Tomanek, B., 2014. Tractography of Meyer's Loop asymmetries. Epilepsy Res. 108, 872-882.

Haxby, J.V., Grady, C.L., Horwitz, B., Ungerleider, L.G., Mishkin, M., Carson, R.E., Herscovitch, P., Schapiro, M.B., Rapoport, S.I., 1991. Dissociation of object and spatial visual processing pathways in human extrastriate cortex. Proc. Natl. Acad. Sci. pathways in human

Hodgkin, A.L., Huxley, A.F., 1952. Propagation of electrical signals along giant nerve fibers. Proc. R. Soc. Lond. B Biol. Sci. 140, 177-183.

Jaekl, P.M., Harris, L.R., 2009. Sounds can affect visual perception mediated primarily by the parvocellular pathway. Vis. Neurosci. 26, 477-486.

Jaekl, P.M., Soto-Faraco, S., 2010. Audiovisual contrast enhancement is articulated primarily via the M-pathway. Brain Res. 1366, 85-92

Johansen-Berg, H., Behrens, T.E., Robson, M.D., Drobnjak, 1., Rushworth, M.F., Brady, J.M., Smith, S.M., Higham, D.J., Matthews, P.M., 2004. Changes in connectivity profiles define functionally distinct regions in human medial frontal cortex. Proc. Natl. Acad. Sci. USA 101, 13335-13340.

Johansen-Berg, H., Della-Maggiore, V., Behrens, T.E., Smith, S.M. Paus, T., 2007. Integrity of white matter in the corpus callosum correlates with bimanual co-ordination skills. Neuroimage 36 (Suppl 2), T16-T21

Johansen-Berg, H., 2010. Behavioural relevance of variation in white matter microstructure. Curr. Opin. Neurol. 23, 351-358.

Kaposvari, P., Bognar, A., Csibri, P., Utassy, G., Sary, G., 2014. Fusion and fission in the visual pathways. Physiol. Res. 63, 625-635.

Klein, J.C., Behrens, T.E., Robson, M.D., Mackay, C.E., Higham, D.J., Johansen-Berg, H., 2007. Connectivity-based parcellation of human cortex using diffusion MRI: establishing reproducibility, validity and observer independence in BA 44/ 45 and SMA/pre-SMA. Neuroimage 34, 204-211.

Legge, G.E., 1978. Sustained and transient mechanisms in human vision: temporal and spatial properties. Vis. Res. 18, 69-81.

Leo, F., Bertini, C., di Pellegrino, G., Ladavas, E., 2008. Multisensory integration for orienting responses in humans requires the activation of the superior colliculus. Exp. Brain Res. 186, 67-77.

MacMillan, N., Creelman, C., 2005. Detection Theory: A User's Guide Routledge. Psychology Press.

Maguire, E.A., Gadian, D.G., Johnsrude, I.S., Good, C.D., Ashburner, J., Frackowiak, R.S., Frith, C.D., 2000. Navigation-related structural change in the hippocampi of taxi drivers. Proc. Natl. Acad. Sci. USA 97, 4398-4403.

Marchant, J.L., Driver, J., 2013. Visual and audiovisual effects of isochronous timing on visual perception and brain activity. Cereb. Cortex 23, 1290-1298.

Mishra, J., Martinez, A., Sejnowski, T.J., Hillyard, S.A., 2007. Early cross-modal interactions in auditory and visual cortex underlie a sound-induced visual illusion. J. Neurosci. 27, 4120-4131.

Neubert, F.X., Mars, R.B., Buch, E.R., Olivier, E., Rushworth, M.F. 2010. Cortical and subcortical interactions during action reprogramming and their related white matter pathways. Proc. Natl. Acad. Sci. USA 107, 13240-13245.
Nichols, T.E., Holmes, A.P., 2002. Nonparametric permutation tests for functional neuroimaging: a primer with examples. Hum. Brain Mapp. 15, 1-25.

Pfordresher, P.Q., Mantell, J.T., Brown, S., Zivadinov, R., Cox, J.L., 2014 Brain responses to altered auditory feedback during musical keyboard production: an fMRI study. Brain Res. 1556, 28-37.

Proverbio, A.M., Calbi, M., Manfredi, M., Zani, A., 2014. Audiovisuomotor processing in the musician's brain: an ERP study on professional violinists and clarinetists. Sci. Rep. 4, 5866 .

Sampaio-Baptista, C., Khrapitchev, A.A., Foxley, S., Schlagheck, T. Scholz, J., Jbabdi, S., DeLuca, G.C., Miller, K.L., Taylor, A. Thomas, N., Kleim, J., Sibson, N.R., Bannerman, D., JohansenBerg, H., 2013. Motor skill learning induces changes in white matter microstructure and myelination. J. Neurosci. 33, 19499-19503.

Saygin, Z.M., Osher, D.E., Koldewyn, K., Reynolds, G., Gabrieli, J.D. Saxe, R.R. 2012. Anatomical connectivity patterns predict face selectivity in the fusiform gyrus. Nat. Neurosci. 15, 321-327.

Scholz, J., Klein, M.C., Behrens, T.E., Johansen-Berg, H., 2009. Training induces changes in white-matter architecture. Nat. Neurosci. 12, 1370-1371.

Shams, L., Kamitani, Y., Thompson, S., Shimojo, S., 2001. Sound alters visual evoked potentials in humans. Neuroreport 12. 3849-3852.

Smith, S.M., Jenkinson, M., Woolrich, M.W., Beckmann, C.F., Behrens, T.E., Johansen-Berg, H., Bannister, P.R., De Luca, M., Drobnjak, I., Flitney, D.E., Niazy, R.K., Saunders, J., Vickers, J. Zhang, Y., De Stefano, N., Brady, J.M., Matthews, P.M., 2004. Advances in functional and structural MR image analysis and implementation as FSL. Neuroimage 23 (Suppl 1), S208-S219. Smith, S.M., Johansen-Berg, H., Jenkinson, M., Rueckert, D., Nichols, T.E., Miller, K.L., Robson, M.D., Jones, D.K., Klein, J.C. Bartsch, A.J., Behrens, T.E., 2007. Acquisition and voxelwise analysis of multi-subject diffusion data with tract-based spatial statistics. Nat. Protoc. 2, 499-503.

Smith, S.M., Nichols, T.E., 2009. Threshold-free cluster enhancement: addressing problems of smoothing, threshold dependence and localisation in cluster inference. Neuroimage 44, 83-98.

Teder-Salejarvi, W.A., McDonald, J.J., Di Russo, F., Hillyard, S.A. 2002. An analysis of audio-visual crossmodal integration by means of event-related potential (ERP) recordings. Brain Res. Cogn. Brain Res. 14, 106-114.

Toosy, A.T., Ciccarelli, O., Parker, G.J., Wheeler-Kingshott, C.A. Miller, D.H., Thompson, A.J., 2004. Characterizing functionstructure relationships in the human visual system with functional MRI and diffusion tensor imaging. Neuroimage 21, 1452-1463.

Ungerleider, L.G., Haxby, J.V., 1994. 'What' and 'where' in the human brain. Curr. Opin. Neurobiol. 4, 157-165.

Watkins, S., Shams, L., Tanaka, S., Haynes, J.D., Rees, G., 2006. Sound alters activity in human V1 in association with illusory visual perception. Neuroimage 31, 1247-1256.

Watkins, S., Shams, L., Josephs, O., Rees, G., 2007. Activity in human V1 follows multisensory perception. Neuroimage 37 , $572-578$.

Wilson, J.T., 1987. Interaction of simultaneous visual events. Perception 16, 375-383. 


\section{II.}


Research report

\section{Aging alters visual processing of objects and shapes in inferotemporal cortex in monkeys}

G. Csete ${ }^{a, b}$, A. Bognár ${ }^{a}$, P. Csibri ${ }^{a}$, P. Kaposvári ${ }^{a}$, Gy. Sáry ${ }^{a, *}$

a Department of Physiology, Faculty of Medicine, University of Szeged, Dóm tér 10, H-6720 Szeged, Hungary b Department of Neurology, Faculty of Medicine, Semmelweis u. 6, H-6725 Szeged, Hungary

\section{A R T I C L E I N F O}

\section{Article history:}

Received 19 June 2014

Received in revised fo

21 November 2014

2014

\section{Keywords:}

Perceptual deficit

Aging

Shape vision

Monkey

\section{A B S T R A C T}

Visual perception declines with age. Perceptual deficits may originate not only in the optical system serving vision but also in the neural machinery processing visual information. Since homologies between monkey and human vision permit extrapolation from monkeys to humans, data from young, middle aged and old monkeys were analyzed to show age-related changes in the neuronal activity in the inferotemporal cortex, which is critical for object and shape vision. We found an increased neuronal response latency, and a decrease in the stimulus selectivity in the older animals and suggest that these changes may underlie the perceptual uncertainties found frequently in the elderly.

O 2014 Elsevier Inc. All rights reserved.

\section{Introduction}

The cognitive functions, including visual perception, declin with age. This deterioration of the perceptual processes can be attributed not only to optical defects such as cataract, glaucoma or presbyopia, but also to neurological aging and dysfunction in higher visual areas. This may have a major impact on the occurrence of home accidents, automobile driving and the quality of life in general (Carter et al., 1997; Desapriya et al., 2014; Kallstrand-Eriksso et al., 2013). Data from psychophysical studies indicate that older people tend to have decreased visual acuity, contrast sensitivity and contour integration (Hutman and Sekuler, 1980; Roudaia et al., 2008; Sekuler et al., 1980). They often demonstrate impairments in visual motion sensitivity, including the perception of apparen motion, and have poorer orientation-judging capabilities (Bennett et al., 2007; Betts et al., 2007; Roudaia et al., 2010) (for a review, see Andersen, 2012). It has been reported that aging also has an impact on form perception and shape discrimination (Habak et al. 2009; McKendrick et al., 2010; Weymouth and McKendrick, 2012) and figure-background separation (Chee et al., 2006), but it is not clear what changes accompany these impairments.

\footnotetext{
* Corresponding author. Tel.: +36 62 545372; fax: + 3662545842 . E-mail addresses: cseteg@gmail.com(G. Csete), annab89@gmail.com (A. Bognár . csibripeter@gmail.com (P. Csibri), kaposvari.peter@med.u-szeged.hu (P. Kaposvári),
} sary.gyula@med.u-szeged.hu (Gy. Sáry).

http://dx,doi.org/10.1016/j.brainresbull.2014.11.00

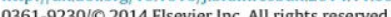

A number of papers have addressed the connection between aging and the decline of visual functions (Liang et al., 2010; Schmolesky et al., 2000; Spear, 1993; Wang et al., 2006; Wang 2001; Yang et al., 2008, 2009a; Yu et al., 2006), but we are not aware of any electrophysiological report concerning the effects of aging on the neuronal activity underlying object and shape vision in a high-level visual area such as the monkey inferotemporal cortex (IT)

The IT is the last unimodal area in the ventral visual stream (Ungerleider and Mishkin, 1982), which receives the visual input mostly from areas V4 and the TEO (Merigan, 1996; Morel and Bullier, 1990; Baleydier and Morel 1992). Cells in the IT best respond to complex, colorful stimuli (Desimone et al., 1984). They might be selective to shapes, i.e., they respond to, or rather respond more strongly to some shapes than to others (Tanaka et al., 1991; Desimone et al., 1984; Gross et al., 1972). More importantly, many of the IT cells keep their selectivity toward the stimuli despite the changes in the retinal position or size (Ito et al., 1995; Logothetis and Pauls, 1995; Rolls and Baylis, 1986; Schwartz et al., 1983; Sato et al., 1980) or the defining visual cue: a particular shape can be defined by differences in luminance, texture, or relative motion without affecting the shape selectivity of the cells (Sary et al., 1993). Thus, these neurons seem to be excellent candidates to process invariant objects and shapes; a mechanism suggested essential for object and shape recognition. The IT is closely associated to perception. Micro-stimulation of the area influences the face non-face categorisation (Afraz et al., 2006), and human perception models 
show similar cell firing patterns as IT neurons (Allred et al., 2005). Furthermore, the use of the binocular rivalry paradigm (Leopold and Logothetis, 1996; Sheinberg and Logothetis, 1997) proves that visual perception and IT activity are closely related. For a recent review of the crucial role of this area and of the ventral pathway in visual perception, see (Tompa and Sary, 2010; Kravitz et al., 2013).

The macaque monkey has been a useful animal model to study human visual perception. Several papers have compared the visual systems of monkeys and humans (Denys et al., 2004; Orban et al., 2004; Tanaka, 1997). If we accept that a possible criterion for homologies in different species is the similarity in function and in retinotopy in the particular visual areas, then it is clear that the ventral cortical pathway that serves object vision is conserved across primates into humans (DeYoe et al.. 1996; Fize et al., 2003; Kourtzi et al., 2003; Kravitz et al., 2013). A homology for IT in humans is probably the posterior part of the inferotemporal cortex or the lateral occipital complex (for reviews, see: Orban et al., 2004; Kravitz et al., 2013), and the investigation of changes in the shape representation of the monkey IT may therefore facilitate an understanding of age-related changes in the human visual system.

The aging of monkeys is similar in many respects to human aging. A 30- to 35-year-old monkey is similar to a human aged 90-95 years (Tigges et al., 1988). On this basis, three human years may be assumed to correspond to one monkey year. Nonhuman primates are ideal for examining central nerve system aging since they share the same neuromorphological pattern as in humans during normal aging (Gallagher and Rapp, 1997; Lowenstine, 2003). Interestingly, the refractory power of the eye tends to decrease, which is appropriately compensated in monkeys (Fernandes et al., 2003; Ooj and Grosvenor, 1995). This supports the assumption that ocular pathology is not critically relevant in the changes in visual function. We hypothesized that changes in the neuronal coding of objects may partially explain the perceptual deficit of aging monkeys and humans.

In the study reported here, we compared data obtained from single-cell recordings from monkeys in two age groups: 7 years and 27-33 years (corresponding to 21 and 81-99 years, respectively, in humans) to determine how the neuronal coding of visual stimuli changes with age in IT.

\section{Methods}

All the participating animals (Macaca mulatta) were engaged in earlier studies in our laboratory. The animals performed a simple fixation task and were exposed to the same set of images at a certain stage during their training (for an example of the stimulus set, see Sary et al., 2006). To compensate for implicit learning, we took our sample from a later phase of the experiments, when the animals could be regarded as experienced or even overtrained. Our behavioral threshold was set to $87 \%$, but at the time of the recording all the animals performed at $>90 \%$ correct level.

Monkeys were examined when they were anaesthetized during the headpost implantation procedure by an ophthalmologist and the same investigation was performed as reported in other papers e.g., Yang et al., 2009a). No major ophthalmoscopical deviations or refractive errors were detected in the lens, vitreous body, vasculature or macula. In addition, monkeys performed at $>90 \%$ correctness in the fixation task that used a few pixel sized fixation point and fixation window of $<1^{\circ}$, which is hardly possible with blurred vision, thus the presence of refractory errors and their corrections were not considered (Fernandes et al., 2003; Ooj and Grosvenor, 1995) It is important to note at this point, that the old animals were purchased and operated on at an old age, thus, it is unlikely that their vision deteriorated rapidly between the surgery and the start of the recordings ( $\sim 2$ months)
The four monkeys that participated in the study weighted between 6 and $9 \mathrm{~kg}$ at the time of the experiments. In the young group, the two monkeys were males and the old animals were 2 females (see Section 4). Prior to their training, the animals underwent two session of aseptic surgery. All surgical procedures were carried out under full anesthesia, induced with an i.m. injection of ketamine (Calypsol; $15 \mathrm{mg} / \mathrm{kg}$ ) and atropine $(0.05 \mathrm{mg} / \mathrm{kg}$ ). An endotracheal tube was inserted into the trachea and anesthesia was maintained with Halothane (1-1.2\%), given in a mixture of $\mathrm{N}_{2} \mathrm{O}$ and $\mathrm{O}_{2}$ in a ratio of $2: 1$, or $0.5 \mathrm{mg} / \mathrm{kg}$ i.v. midazolam (Dormicum). An i.v. line was inserted for continuous access and physiological saline was given to compensate for fluid loss. Before the surgical procedure, a preventive dose $(250 \mathrm{mg}$ ) of the antibiotic ceftriaxone (Rocephin) was given. The incision was infiltrated with local anesthetic (Procaine). Nalbuphin and nonsteroidal anti-inflammatory drugs were administered to the animals postoperatively. The arterial oxygen saturation, expired $\mathrm{CO}_{2}$ level, heart rate and alveolar concentration of the inhalation narcotic were monitored continuously throughout the surgery. A stainless steel headpost was fixed to the head to keep the animal's head stable, and a search coil was implanted under the conjunctiva (Judge et al., 1980) to enable recording of the eye position.

Before the implantation of the registration chamber, all animals underwent T1 and T2 weighted MR acquisitions. Stereotaxic coordinates and individual MR images were used during the surgical procedure to optimize the position of the registration chamber (attitude: 23; latitude: 17). After the surgery, X-ray images were made with the lowered electrode to test the proper location of the electrodes.

During recording, the alternation of white and gray matter during advancing of the electrode, the selectivity for complex colo stimuli, the typical response latency values (Schmolesky et al., 1998; Sáry et al., 2006) are a hint for being in the right location. Finally, after the examination, the brains were removed and frozen sections were made to locate the electrode tracts, which pointed to area TE, the lower bank of STS and the lateral part of TE.

The animals performed a fixation task. First, a red fixation point was presented on the monitor (distance: $57 \mathrm{~cm}$ ) for $500 \mathrm{~ms}$, followed by a gray background $(500 \mathrm{~ms})$ and then by the stimulus $(500 \mathrm{~ms})$. For each cell, 20 stimuli were used, showing colo reproductions of real world 3D objects or geometric shapes (Sary et al., 2004). For each of the stimuli at least 10 successful trials (and recordings) were collected in a semi-random fashion. Trials were considered successful if the animal did not break fixation all through the trial (fixation window $\sim 0.5^{\circ}$ ). Trials were machine paced, the inter-trial interval was set to $1 \mathrm{~s}$. This way, collecting all the required responses lasted for about 7-8 min, depending on the number of unfinished trials. Only correct trials were included in the off-line analysis. Once a responsive cell had been isolated, a set of effective and non-effective stimuli were chosen and the neurona responses were recorded with the use of these stimuli. This allowed us to construct stimulus-preference tuning curves, a feature characteristic of IT cells. The neuronal activity from the IT was recorded by using standard electrophysiological methods.

Cellular activity was analyzed off-line by means of Statistica (Statsoft) and MATLAB (Math Works). All procedures relating to the surgery and training of the animals conformed fully to the NIH standards and had been approved in advance by the Ethical Council of the University of Szeged.

To characterize the recorded neuronal responses, the following parameters were used:

- Baseline: the mean activity in a time window of $400 \mathrm{~ms}$ preceding stimulus onset.

- The response latency time: the time from stimulus onset to the start of the neuronal activity. We used the Poisson spike train 
analysis method, which has previously proved to be reliable in different experimental paradigms (Hanes et al., 1995: Sary et ab. 2006; Thompson et al., 1996).

- The net firing rate: the mean baseline activity subtracted from the mean overall activity in a 400-ms time window starting $100 \mathrm{~m}$ after stimulus onset. Baseline activity does not change immediately after stimulus presentation, thus, if net responses are considered, like in this study, a certain time after stimulus onset might be omitted. The mean response latency time in IT being $100 \mathrm{~ms}$, it is a general convention to leave out the first $100 \mathrm{~ms}$ or so starting at stimulus onset (e.g., Sáry et al., 1993; Missal et al. 1999; Op de Beeck et al., 2001; Tamura and Tanaka, 2001). This procedure does not have influence on the results, in contrary, removes some noise from the data.

- The evoked-to-spontaneous ratio: a value calculated by dividing the net firing rate by the baseline activity. This shows how much the stimulus-related activity differs from the background activity.

- Fano factor: the standard deviation of probability distribution of the spike counts. This is one of the most widely used statistical parameters to describe the variability of the spike trains (Churchland et al., 2010).

The following factors are considered special to neurons which play roles in the sensory perception and discrimination of, fo example, the speed and direction of movement or shapes.

- The selectivity index ( $\mathrm{SI}$ ): this is used to describe the stimulus preference of cells.

$$
\mathrm{SI}=\frac{R_{\max }-R_{\min }}{R_{\max }+R_{\min }}
$$

where $R_{\max }$ is the maximal and $R_{\min }$ is the minimum response to a particular stimulus set. The closer the value is to 1 , the better the cell discriminates between a preferred and a non-preferred stimulus.

- The depth of selectivity index (DSI). Long-term recordings have indicated that the cellular responses can best be characterized by a variable derived from the median of the firing rate (Bonda et al., 2009). In the optimal case, i.e. if the cell shows a stimulus preference, the value is 1 or close to it. It is a normalized variable, with the greatest response taken as 1 and the median of the responses being subtracted from it:

$\mathrm{DSI}=\frac{n-\sum_{i=1, n} R_{i} / R_{\max }}{n-1}$,

where $R_{i}$ is the $i$ th response to the $n$ stimuli, while $R_{\max }$ is the max imum response. The value ranges from 0 to 1 , where the highest value indicates that the examined cell reacts to only one stimulus. - The sparseness index (SP): the ratio between the used and effective stimuli, i.e. the "tail" of the distribution of the cellula responses to the stimulus set (Rolls and Tovee, 1995).

$\mathrm{SP}=\frac{\left(\sum_{i=1, n} R_{i} / n\right)^{2}}{\sum_{i=1, n} R_{i}^{2} / n}$,

where $R_{i}$ is the $i$ th response from the responses to a stimulus se containing $n$ stimuli. The value can range between 0 and 1 . A small SP value means that there are only a few stimuli in the set which can evoke large responses (i.e. there is a stimulus preference) while a large SP values means that most of the stimuli can trigger cellular responses which do not differ too much.

\subsection{Statistics}

Nonparametric tests were used throughout the analysis. To verify the differences between groups, we performed a Mann-Whitney $U$ test. Results $(U)$ were compared with the corresponding value of the $\chi^{2}$-table at the same degree of freedom (df). Results were taken as significant if the type I error was less than $5 \%(p<0.05)$. All values are presented with the median and the interquartile range in the text and in the table. Correction for multiple comparisons was applied according to the Bonferroni method. In some cases, however, we present both corrected ( $p_{\text {corr }}$ ) and uncorrected values, as we consider that the Bonferroni correction is too conservative and could mask relevant results. For this reason a nonparametric permutation test was implemented (number of permutations $=5000$ Cluster analysis (Ward method) was used to reveal the relationship between possible groups among the variables.

\section{Results}

We recorded a total of 288 neurons from $4 \mathrm{M}$. mulatta monkeys to investigate the functional consequences of neuronal aging Two groups of data were formed for comparison: a young group ("Young", 2 monkeys, aged 7 years, number of cells: 221) and an old group ("Old", 2 monkeys aged 27 and 33 years, respectively, number of cells: 67). We present two representative histograms in Fig. 1, and the statistically relevant data in Table 1.

First to have an initial analysis of our data set we ran a cluster analysis to determine whether our data form groups according to the age or not. Baseline activity, as the raw data with no modulation was chosen to explore the internal pattern of the groups. Second, latency data were also used, as our hypothesis was that this is a primary measure of cell aging (Kuba et al., 2012; Wang et al., 2005). The cluster analysis revealed two main clusters, one comprising the data on the 2 Old animals and the other the data on the Young ones.

In the next step, we compared the data on all the registered cells by means of the Mann-Whitney $U$ test. The baseline of firing rate did not significantly differ in the 2 groups: $6.00(2.81-9.69)$ spikes/s in the Young, and $5.23(2.50-10.27)$ spikes/s in the Old animals, respectively.

The evoked-to-spontaneous ratio was $3.63(2.19-6.10)$ in the Young and $3.26(2.34-6.75)$ in the Old group. Once again, there was no significant difference between the groups.

The Fano factor did not indicate differences between the groups either: $1.26(0.76-1.85)$ and $1.31(0.84-2.00)$ for the Young and Old groups, respectively.

The net firing rates were likewise not different: 15.67 (8.33-25.33) spikes/s vs. 14.33 (8.33-24.66) spikes/s, respectively, in the Young-Old comparison.

On the other hand, the latency revealed a difference between the Young and Old groups (Table 1): $128.73(96.88-161.86) \mathrm{ms}$ and 147.20 (124.54-179.83) ms, respectively; $U=4101.00 ; p_{\text {corr }}<0.05$.

SI too demonstrated a difference $\left(U=4810.50 ; p_{\text {corr }}<0.001\right)$ in the Young vs. Old comparison: $0.89(0.69-0.97)$ and 0.76 (0.47-0.90), respectively.

The SP values for the Young-Old groups (Table 1) were 0.47 $(0.28-0.73)$ and $0.75(0.56-0.90)$, respectively, this difference was significant, according to the Mann-Whitney test $(U=4245.50$; $p_{\text {corr }}<0.001$ ).

DSI similarly pointed to a group difference in the Young-Old comparison: $0.60(0.44-0.79)$ vs. $0.46(0.31-0.68)$, respectively: $U=5197.00 ; p_{\text {corr }}<0.01$.

To reduce the biasing effects of unequal samples, we repeated the analysis on 22 randomly chosen cells from each animal, i.e., 44 

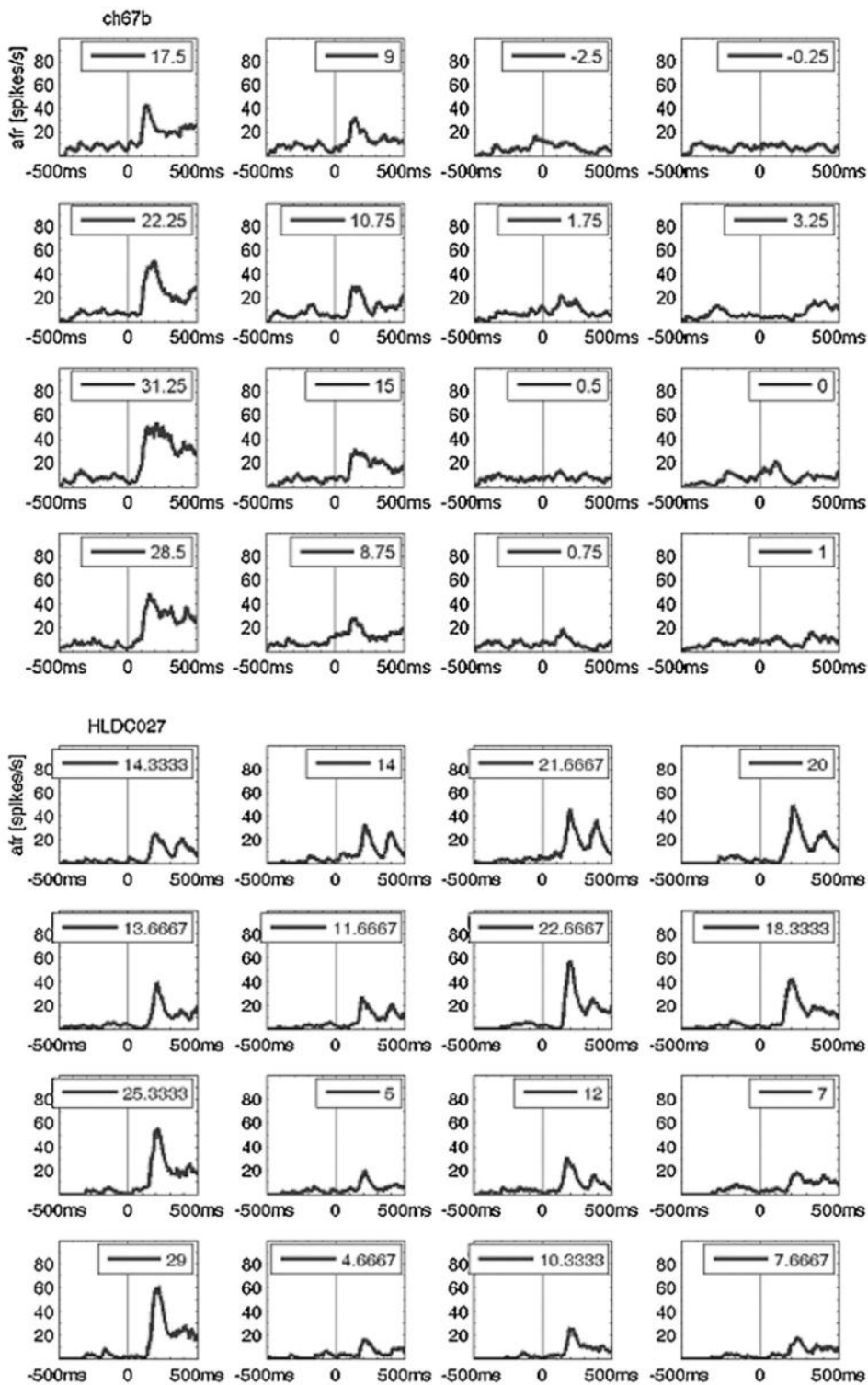

Fig. 1. Peristimulus time histograms of two cells recorded from $\Pi$. Upper panel: Young animal, bottom panel: Old animal. Each window contains the responses (minimum 10 the given to one of the stimull of cells from each age group. Cells were selected from that stage of the experiments when the animals were considered overtrained. During this phase, the animals worked on a daily basis, achieving a $>90 \%$ performance rate in the task, and the effect of attentional

As in the analysis involving all the cells, the response latency values of the Young and Old data were statistically different: 124.85 $(97.00-161.74) \mathrm{ms}$ and $154.51(129.33-178.64) \mathrm{ms}$, respectively $\left(U=567.00 ; p_{\text {corr }}<0.01\right)$ (Fig. 2). Also, the SI values pointed to a significant difference between the groups $(U=713.50 ; p<0.034)$ 
Table 1

Statistical values for the Young vs. Old comparison (median + interquartile range).

\begin{tabular}{lccc}
\hline & Young & Old & $p$-Value \\
\hline Latency (ms) & $124.85(97.00-161.74)$ & $154.51(129.33-178.64)$ & $<0.01$ \\
Selectivity index & $0.90(0.69-0.97)$ & $0.81(2.51-0.92)$ & $<0.027$ \\
Sparseness index & $0.46(0.29-0.73)$ & $0.75(0.51-0.88)$ & $<0.05$ \\
Depth of selectivity index & $0.61(0.46-0.80)$ & $0.51(0.37-0.71)$ & $<0.02$ \\
\hline
\end{tabular}
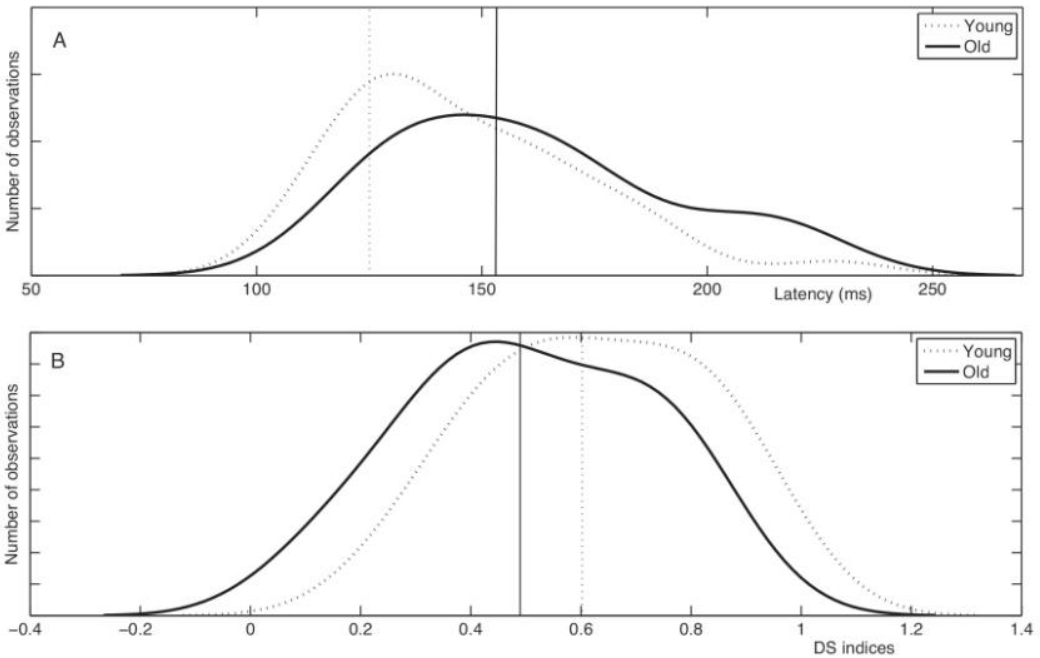

Fig. 2. Graphs showing the distribution of latency data (panel A) and the DSI values (panel B). Dotted lines on both graphs show data and median values of the Young. continuous line the data and median values of the Old group, respectively.

before the Bonferroni correction, which disappeared after it ( $\left.p_{\text {corr }}<0.27\right)$. Since in our opinion, this difference is essential for understanding neuronal aging and the decrease in perceptual performance, we rerun this comparison with the Monte-Carlo method in addition to the Bonferroni correction ( $p_{\text {permutation }}<0.027$ ). The neuronal selectivity values in the two groups were: 0.90 $(0.69-0.97)$ vs. $0.81(0.51-0.92)$. As for the DSI values, the same procedure masked the result $\left(U=670.00 ; p<0.013, p_{\text {corr }}<0.10\right)$. However, after randomization, the differences were significant $(<0.02)$ (Fig. 2).

The SP parameters, were significantly different $(U=587.00$ $\left.p_{\text {corr }}<0.05\right)$ : $0.46(0.29-0.73)$ vs. $0.75(0.51-0.88)$, in the Young and Old groups, respectively.

\section{Discussion}

Naturally, the best way to study age-related differences would be therefore long-term monitoring (>10years) of the same subjects, but that is technically not feasible. We compared results obtained from awake, behaving monkeys belonging in two appreciably differing age groups, young and old, while the animals performed the same task, with the identical stimulus set. Differences emerged between the different age groups in the response latencies and selectivity indices.

The gender combination may cast some doubt on the results: however, we might be able to provide a satisfactory explanation, why gender is not an issue. The baseline activity data that reflected the resting state of the neurons were chosen to demonstrate that data form internal groups (cluster analysis and Ward method); the same procedure was repeated with the latency values. Both results show that data is separated by age and not gender. There is no doubt that sexual dimorphism exists in monkeys like in the neuroendocrine system or in higher cognitive performances, and it is also known that females are less affected e.g. by ischemic lesions but there is no data concerning the basic sensory processing. We did an extensive search in the literature regarding electrophysiological analysis of the visual system in the monkeys, and did not find any relevant proof for gender related differences. Most importantly, our older female monkeys did not show any sign of cyclic ovarian activity during their stay in our laboratory.

\subsection{Baseline activity}

The baseline activity reported here is in the normal range (e.g. Mirpour and Esteky, 2009). Since response levels and selectivity values might depend on the stimulus set used, it does not make sense to compare them with data available in the literature.

The baseline activity did not appear to change with aging in these animals, whereas others have reported an elevation in baseline activity in older animals (Hua et al., 2006; Schmolesky et al., 2000; Yang et al., 2008). Juarez-Salinas et al. (2010) also reported an increase of baseline activity in the auditory cortex. This, an other possible differences between our and other studies, may be explained by the methodological differences: our animals were awake and performed a fixation task, while the recordings in othe studies were made on anesthetized animals (cats, Hua et al., 2006, 
monkeys, Yang et al., 2008). We compared activity levels of the IT, while other studies compared activity in the striate cortex (Schmolesky et al., 2000) or in area MT (Yang et al., 2008). Our animals performed a simple fixation task with biologically irrelevant stimuli. The task was machine-paced, which puts some load an attention, thus small individual differences in baseline activity might disappear. Although a stimulus preference of neurons and tolerance against identity preserving changes (e.g. stimulus size and position) have been observed in anesthetized animals (Hung et al., 2005), it is well known that recording in awake animals has various benefits. It is not necessary to consider the occasionally undulating level of anesthesia; while the cellular response rate is generally higher (this may improve the signal to noise ratio), more explicit (Tamura and Tanaka, 2001; Tanaka et al.., 1991).

\subsection{Neuronal responsivity}

Previous papers have reported an age-related changes in the neuronal response levels in V1, V2 and middle-temporal complex (MT) in monkeys (Leventhal et al., 2003; Schmolesky et al., 2000; Wang et al., 2005; Yang et al., 2009b), which also affected the signal to noise ratio of the responses. We did not observe any significant change in the responsivity of the neurons between the Young and Old groups, and the evoked-to-spontaneous ratio was not affected either. Since we know of no study in which the age-related activity changes in V1, V2, MT and IT were compared in the same animal, we cannot explain this controversy. It may possible be caused by the differences between awake and anesthetized animals.

A number of neuroimaging studies have reported age-related changes in cerebral activity in human subjects that resemble those observed in our study. An object-identifying PET study demonstrated a decreased inferior temporal regional cerebral blood flow (rCBF) level (Schiavetto et al., 2002). Similarly, an age-related attenuation in blood-oxygen-level dependent (BOLD) signal was detected in the temporal lobe during object recognition (Ramsoy et al., 2012). Other reports have shown that a possible impairment in areas needed for the performance of a task may be compensated by an increased activity in the basal ganglia (Madden et al.. 2004) or frontal cortical regions (Grady et al., 1994. Gutchess et al., 2005 , 2007). However, as the coupling of the BOLD signal to neuronal activity has been reported to change with age, care must be taken when young and old subjects are compared in imaging studies (D'Esposito et al., 1999).

\subsection{Increased latency}

The typical latency values in the IT are a bit longer than $100 \mathrm{~ms}$, depending on the method (Schmolesky et al., 1998; Sáry et al., 2006) but values around $150 \mathrm{~ms}$ have also been reported (Tamura and Tanaka, 2001). In this paper we used the method based on the Poisson spike train analysis (Hanes et al., 1995) which can reliably detect small differences in the neuronal response latency time(Sáry et al., 2006).

It is not possible to explain the origin of the delayed latency values from the data we report here. Some authors argue for a generalized slowing in processing speed during aging (Salthouse, 1996) and many papers describe age-related increase in response latencies. Increased response latency was recently observed in rats (Wang et al., 2006). Evoked potential studies revealed an increase in the peak latency time of $\mathrm{P} 3$ in human subjects during both passive and active tasks, which indicates a slowing-down of cognitive tasks at older ages (Morgan and Murphy, 2010). Similarly, the response latency differences between good and poor performers increased with advancing in age (Riis et al., 2008), which indicates the agedependent factors in visual processing during normal aging. In a
PET experiment, a face-matching task demonstrated an increase in reaction times (Grady et al, 1994). The underlying mechanism is not clear as no decrease was found in the number of retinal ganglion cells (Kim et al., 1996) or lateral geniculate body cells (dLGB) (Ahmad and Spear, 1993) during aging. On the other hand, changes were discovered in layer 3 of V1, but they did not affect the electrophysiological properties (Luebke et al., 2013), and no significant decreases were found in the total numbers of V1 neurons and glias (Giannaris and Rosene, 2012). This might direct attention to age-related functional changes. Recent results suggest that myelin breakdown can affect the processing speed (Lu et al. 2011, 2013), and we hypothesize that this might be the reason for the observed increased response latencies. This idea is supported by the fact that there is a strong correlation between age-related myelin breakdown and cognitive impairment in monkeys (Peters and Sethares, 2002). Moreover, the axonal conduction velocity is decreased in aged cats (Xi et al., 1999). The electrophysiological properties change together with the age-associated deterioration of the myelin in the cortex (Luebke et al., 2010). This slowingdown could be a general phenomenon in the nervous system, but it certainly affects the corresponding visual cortical areas as well (Peters et al., 2000). We must note that there is also data available that presents decreased latency in older monkeys in the auditory cortex (Engle and Recanzone, 2013). However, the latency values reported in the literature are difficult to compare because of the methodological differences (species, recording site, anaesthetized vs., awake, passive vs, task performing, etc.) not to speak of the many existing methods to define them (Schmolesky et al.., 1998; Sáry et al., 2006).

\subsection{Change in stimulus selectivity}

In general, IT cells react well to complex visual stimuli, but to a different degree. The differentiation among stimuli is reflected in the different response levels (stimulus preference), which is considered to be the basic property of IT cells. A common model for coding stimuli in IT consists of a limited number of cells, showing differen responses to different stimuli (Tanaka et al., 1991; Desimone et al. 1984: Gross et al., 1972). Thus, objects are coded by the activity pattern of many neurons, in a population code, where some cells respond and some do to a less degree or not to the stimuli in a given stimulus set. A cell, showing vigorous responses to certain stimuli but not responding to others sharpens the stimulus preference.

The key to the perception of different aspects of the visual stimulus thus is probably a set of neurons with stimulus selectivity (or more precisely, stimulus preference) for a particular parameter (speed, direction, orientation, shape, color, etc.), and thus an altered preference or influencing the firing pattern of these neurons will have an impact on the perception of the particula item (Salzman and Newsome, 1994). Minor functional age-related changes have been observed in the dLGB (Spear et al., 1994), and in the higher stages of the visual hierarchy the changes are more expressed (Schmolesky et al., 2000). It is suggested, for instance, that V2 is more affected than V1 (Yu et al., 2006), similarly to higher levels (Liang et al., 2010). Indeed, there is a clear distinction between the aging of simple and complex cells in V1 (Liang et al., 2012). Changes in speed representation and increased response variability in the MT, might well explain the altered speed perception in aging humans; such findings were interpreted by supposing a decreased number of glycinergic neurons (Yang et al., 2009a,b) but a decreased number of interneurons in the cortex has also been reported (Coleman and Flood, 1987). The shape selectivity in IT receptive fields is formed by GABAergic neurons (Wang et al., 2002). It might be that the number of these neurons decreases with age, and that this leads to an altered shape preference. Further, microstructural changes including a deterioration of the 
myelin sheets (Inano et al., 2011) and changes in the white matter (fractional anisotropy in the occipital region) (Van Impe et al., 2012) were postulated to be possible predictors of sensory degeneration. Others have reported an accumulation of microglia and oligodendrocytes in the primary auditory and visual cortices (Tremblay et al., 2012). A possible explanation was suggested for the impairment of effectivity in sensory-perceptual processing following the finding of decrease in occipital gray matter and activity (Kalpouzos et al., 2012). A reduced gray matter volume and decreased discrimination capability reflecting aging of the brain have likewise been reported (Carp et al., 2010). Human experiments clearly demonstrated age-related changes in shape perception (McKendrick et al., 2010; Weymouth and McKendrick, 2012) and visuospatial memory (Carp et al., 2010), though the results appear to depend strongly on the experimental methods used (Habak et al., 2009).

It seems, that from middle age on, the changes in neuronal activity that result in perceptual deficits become increasingly explicit. This was recently illustrated by Riis et al. (2008). Unfortunately. we had only one animal in this age group, so these data are not presented here.

Regarding age-related deficits, several of the above papers sug gested a structural deterioration of the neuronal mechanisms supporting visual perception. On the other hand, many finding lend support to the hypothesis that perceptual impairments in aging are due to functional rather than morphological changes, which affect the communication between neurons (Hua et al., 2006: Leventhal et al., 2003; Mendelson and Wells, 2002; Schmolesky et al., 2000; Wang et al., 2006; Yang et al., 2008). Unfortunately our findings do not allow a decision between the mechanisms mentioned above; we have merely demonstrated a phenomenon that has previously not been described.

In summary, we have reported an increase in response latency and a worsening of the stimulus selectivity in the monkey homolog of the human LOC, which might explain, at least partially the uncertainties in visual perception observed in the elderly.

\section{Conflict of interest}

There are no actual or potential conflicts of interest.

\section{Acknowledgements}

This research was supported by Országos Tudományos Kutatási Alap (OTKA) K83671 awarded to Gy. Sáry. The authors thank P. Liszli, G. Dosai and L Rácz for technical support and for taking care of the animals; J. Navracsics and D. Durham for proof-reading the manuscript. This research was supported by the European Unio and the State of Hungary, co-financed by the European Social Fund in the framework of TÁMOP-4.2.4.A/2-11/1-2012-0001 'National Excellence Program'

\section{References}

Afraz, S.R., Kiani, R., Esteky. H., 2006. Microstimulation of inferotemporal cortex influences face categorization. Nature 442, 692-695.

Ahmad, A., Spear, P.D., 1993. Effects of aging on the size, density, and number of rhesus monkey lateral geniculate neurons. J. Comp. Neurol. 334, 631-643.

Allred, S., Liu, Y.., Jagadeesh, B., 2005. Selectivity of inferior temporal neurons for
realistic pictures predicted by al gorithms for image database navigation. J. Neurealistic pictures predicte
rophysiol. $94,4068-4081$

Andersen, G.J., 2012. Aging and vision: changes in function and performance from Andersen, G.J., 2012. Aging and vision: changes in function and perfor
optics to perception. Wiley Interdiscip. Rev. Cogn. Sci. 3, 403-410. leydier to Mereption. Wiley interdiscip. Ralmocortical pas etal and inferotemporal cortex in macaque monkey. Vis. Neurosci. 8, 391-405. Bennett, P.J., Sekuler, R., Sekuler, A.B., 2007. The effects of aging on motion detectic and direction identification. Vis, Res. 47, 799-809,

setts, LR., Sekuler, A.B., Bennett, P.J., 2007. The effects of aging on orientation discrimination. Vis. Res, 47, 1769-1780.
Bondar, I.V., Leopold, D.A., Richmond, B.J., Victor, J.D., Logothetis, N.K., 2009. Longerm stability of visual pattern selective responses of monkey temporal lobe neurons. PLOS ONE 4, e8222.

Carp. J., Gmeindl, L, Reuter-Lorenz, P.A., 2010. Age differences in the neural representation of working memory revealed by multi-voxel pattern analysis. Front Hum. Neurosci. 4, 217

Carter, S.E., Campbell, E.M., Sanson-Fisher, R.W., Redman, S., Gillespie, W.J., 1997. Environmental hazards in the homes of older people. Age Ageing 26, 195-202,
Chee A., Leshikar. E., Park, D., 2006. Age-related changes in object processing and contextual binding revealed using fMR adaptation. J. Cogn. Neurosci. 18, 495-507. Churchland, M.M. Y. Yu, B.M.., Cunningham, J.P., Sugrue, L. ., Cohen, M.R., Corrado, G.S Newsome, W.T., Clark, A.M., Hosseini, P.., Scott, B.B., Bradley. D.C., Smith, M.A. Kohn, A., Movshon, J.A., Armstrong, K.M., Moore, T., Chang, S.W., Snyder, LH. Lisberger, S.G., Priebe, N.J., Finn, I.M., Ferster, D., Ryu, S.I., Santhanam, G., Sahani, M., Shenoy, K.V.,2010. Stimulus onset quenches ne,

com aging and Alzheimer's disease. Neurobiol, Aging 8, 521-545,

enys, K. Vanduffel, W., Fize, D., Nelissen, K. Peuskens, H. Van Essen, D. Orban, G.A. 2004. The processing of visual shape in the cerebral cortex of human and nonhuman primates: a functional magnetic resonance imaging study. J. Neurosci. 24, 2551-2565.

Desapriya, E., Harjee, R., Brubacher, J., Chan, H., Hewapathirane, D.S., Subzwari, S Pike, L., 2014. Vision screening of older drivers for preventing road trafficinjuric of inferior temporal neurons in the macaque. J. Neurosci. 4, 2051-2062.
Dsposito, M..Zarahn, E.., Aguirre, G.K., Rypma, B., 1999. The effect of normal aging o the coupling of neural activity to the bold hemodynamic response. Neuroimage
the $10,6-14$.

DeYoe, E.A., Carman, G.J., Bandettini, P., Glickman, S.. Wieser, J., Cox, R., Miller, D. Neitz. J., 1996. Mapping striate and extrastriate vis

Engle, J R Recanzone $\mathrm{CH}$ 2013. Charaterizing spatial hening functions of neurons in the auditory cortex of young and aged monkeys: a new perspective on old data. Front. Aging Neurosci. 4, 36.

Fernandes, A., Bradley, D.V., Tigges, M., Tigges, J., Herndon, J.G., 2003. Ocular measurements throughout the adult life span of rhesus monkeys, Invest. Ophthatmol. Vis. Sci. 44, 2373-2380.

Fize, D., Vanduffel, W., Nelissen, K., Denys, K., Chef d'Hotel, C., Faugeras, O., Orban. .A., 2003. The retinotopic organization of primate dorsal V4 and surround Neurosci. 23, 7395-7406

Gallagher, M., Rapp, P.R., 1997. The use of animal models to study the effects of aging on cognition. Annu. Rev. Psychol. 48, 339-370.

Giannaris, E.L., Rosene, D.L., 2012. A stereological study of the numbers of neurons and glia in the primary visual cortex across the lifespan of male and female rhesus monkeys. J. Comp. Neurol. 520, 3492-3508.

Grady, C.L., Maisog. J.M., Horwitz, B., Ungerleider, LG., Mentis, M.J., Salerno, J.A Pietrini, P.., Wagner, E., Haxby. J.V... 1994. Age-related changes in cortical bloo flow activation

Gross, C.G., Rocha-Miranda, C.E., Bender, D.B., 1972. Visual properties of neurons in inferotemporal cortex of the macaque. J. Neurophysiol. 35, 96-11

utchess, A.H., Welsh, R.C., Hedden, T., Bangert, A., Minear, M., Liu, L.., Park, D.C 005. Aging and the neural correlates of successful picture encoding: frontal actiations compenste for decresed mediletemporat activity. J Cogn Neurost.

Gutchess, A.H., leuji, Y., Federmeier, K.D.. 2007. Event-related potentials reveal age differences in

Habak, C., Wilkinson, F., Wilson, H.R., 2009. Preservation of shape discrimination in aging. J. Vis. 9 (1), 1-8.

anes, D.P.. Thompson, K.G..S Schall, J.D., 1995. Relationship of presaccadic activity in frontal eye field and supplementary eye field to saccade initiation in macaque: Poisson spike train analysis, Exp. Brain Res. 103, 85-96.

Hua, T., Li, X., He, L., Zhou, Y.. Wang, Y., Leventhal, A.G., 2006. Functional degradation of visual cortical cells in old cats. Neurobiol. Aging 27, 155-162.

Hung, C.P., Kreima. Poggio, T., DiCarlo,J..., 2005. Fast readout of object identity from macaque inferior temporal cortex. Science 310, 863-866. tol, $35,700-706$.
.

Inano, S., Takao, H., Hayashi, N.. Abe, O., Ohtomo, K., 2011. Effects of age and gende on white matter integrity. AJNR Am. J. Neuroradiol, 32, 2103-2109.

M. Tamura, H., Fujita, L., Tanaka, K., 1995. Size and position invariance of neuronal responses in monkey inferotemporal cortex. J. Neurophysiol. 73

. serial processing in the spatial auditory cortical pathway is degraded by natura aging. J. Neurosci. 30, 14795-14804

Judge, S.J., Richmond, B..., Chu, F.C., 1980. Implantation of magnetic search coils fo measurement of eye position: an improved method. Vis. Res. 20, 535-538. Kallstrand-Eriksson. J., Baigi. A., Buer, N., Hildingh, C., 2013. Perceived vision-relate quality of life and risk of 
Kalpouzos, G., Persson, J., Nyberg, L., 2012. Local brain atrophy accounts for functional activity differences in normal aging. Neurobiol. Aging 33, 623e1-623e13. Kim, C.B., Tom, B.W., Spear, P.D.. 1996. Effects of aging on the densities, numbers, and sizes of retinal ganglion cells in rhesus monkey. Neurebiol. Aging $17,431-438$.
(n) ourtzi, Z. Tolias, A.S., Altmann, C.F., Augath, M., Logothetis, N.K., 2003. Integration
of local features into global shapes: monkey and human FMRI studies. Neuron

Kravitz.J.D., Saleem, K.S., Baker, C.I., Ungerleider, L.G., Mishkin, M., 2013. The ventral visual pathway: an expanded neural framework for the processing of object n. Sci. $17,26-49$.

Kuba, M., Kremlacek, J.. Langrova, J., Kubova, Z, Szanyi, J., Vit, F., 2012. Aging effect in pattern, motion and cognitive visual evoked potentials. Vis. Res. 62, 9-16. Leopold, D.A., Logothetis, N.K., 1996. Activity changes in early visual cortex reflect monkeys percepts during binocular rivalry. Nature 379, 549-553.

Leventhal, A.C., Wang, Y., Pu, M.., Zhou, Y., Ma, Y., 2003. GABA and its agonists improved visual $Y$. affects the direction selectivity of MT Yeells in rhesus monkeys, Neurobiol. Aging $31,863-873$.

Liang, Z., Li, H., Yang, Y., Li, G.. Tang, Y., Bao, P., Zhou, Y., 2012. Selective effects of aging on simple and complex cells in primary visual cortex of rhesus monkeys.

Brain Res. 1470, 17-23.
Logothetis, N.K., Pauls, J., 1995. Psychophysical and physiological evidence for viewer-centered object representations in the primate. Cereb. Cortex 5 ,

owenstine, LJ., 2003. A primer of primate pathology: lesions and nonlesions, Toxicol. Pathol. 31 (Suppl.), 92-102.

Lu, P.H., Lee, G. Raven, E.P. Tingus, K., Khoo, T., Thompson, P.M., Bartzokis, G. 2011. Age-related slowing in cognitive processing speed is associated with myelin integrity in a very healthy elderly sample. J. Clin. Exp. Neuropsychol.

L, P.H., Lee, G.J., Tishler, T.A., Meghpara, M., Thompson, P.M., Bartzokis, G., 2013. Myelin breakdown mediates age-related slowing in cognitive processing speed

uebke, J.. Barbas, H., Peters, A., 2010. Effects of normal aging on prefrontal area 46

in the rhesus monkey. Brain Res. Rev. 62, 212-232.
. P.R., Peters, A., 2013. Age-related changes to layer 3 pyramidal cells in the rhesus

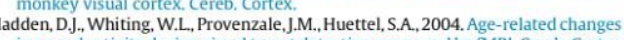
in neural activity during visual target detection measured by fMRI. Cereb. Cortex

McKendrick, A.M., Weymouth, A.E., Battista, J.. 2010. The effect of normal aging on closed contour shape discrimination. J. Vis, 10 (1), 1-9.

Mendelson, J.R. Wells, E.F., 2002. Age-related changes in the visual cortex. Vis. Res, 42, 695-703.

Merigan, W.H., 1996. Basic visual capacities and shape discrimination after lesions of extrastriate area V4 in macaques. Vis. Neurosci. 13, 51-60.

Mirpour, K.. Esteky. H.. 2009. State-dependent effects of stimulus presentation duration on the temporal dynamics of neural responses in the inferotemporal cortex of macaque monkeys. Neurophysiol, 102, 1790-1800,

inferio tem Morel, A., Bullier, J., 1990. Anatomical segregation of two cortical visual pathways in the macaque monkey. Vis. Neurosci. 4, 555-578,

Morgan, C.D., Murphy, C., 2010. Differential effects of active attention and age on event-related potentials to visual and olfactory stimuli. Int. J. Psychophysiol. 78 ,

Ooj, C.S., Grosvenor, T., 1995. Mechanisms of Emmetropization in the Aging Eye. Optometry H. Wasemins. J Vog

sent low-dimensional configuration, R., 2001. Inferotemporal neurons repre$4,1244-1252$.

Orban, G.A., Van Essen, D., Vanduffel, W., 2004. Comparative mapping of higher

visual areas in monkeys and humans. Trends Cogn. Sci. 8, 315-324.
Peters, A., Sethares, C., 2002. Aging and the myelinated fibers in prefrontal cortex and corpus callosum of the monkey. J. Comp. Neurol. 442, 277-291.

eters, A., Moss, M.B., Sethares, C., 2000. Effects of aging on myelinated nerve fibers in monkey primary visual cortex. J. Comp. Neurol. 419, 364-376. Baare, W.., Paulson, O.B., Jernigan, T.L., Siebner, H.R., 2012. Healthy aging attenuates task-related specialization in the human medial temporal lobe. Neurobiol. Aging 33, 1874-1889.

Riis, J.L, Chong, H., Ryan, K.K., Wolk, D.A., Rentz, D.M., Holcomb, P.J., Daffner, K.R., 2008. Compensatory neural activity distinguishes different patterns of norma

Rolls, E.T., Baylis, G.C., 1986. Size and contrast have only small effects on the responses to faces of neurons in the

Rolls, E.T., Tovee, M.J., 1995. Sparseness of the neuronal representation of stimuli in the primate temporal visual cortex. J. Neurophysiol. $73,713-726$.

Roudaia, E., Bennett, P.J., Sekuler, A.B., 2008. The effect of aging on contour integration. Vis. Res. 48, 2767-2774. udaia, E., Bennett, P.J., Sekuler, A.B., Pilz, K.S.,. 2010. Spatiotemporal properties of
apparent motion perception and aging.J. Vis. 10, 5. athouse, T.A., 1996. The processing-speed theory of adult age differences in cogition. Psychol. Rev. 103, 403-428. zman, C.D., Newsome, W. 1994.

decision. Science 264, 231-237.

Sary, G., Vogels, R., Orban, G.A., 1993. Cue-invariant shape selectivity of macaque inferior temporal neurons. Science 260, 995-9.97.

ry, G., Chadaide, Z., Tompa, T., Kovacs, G., Koteles, K., Boda, K., Raduly. L, Benedek, . 2004. Relationship between stimulus complexity and neuronal activity in the $1-12$

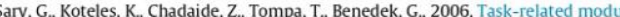
lation in the monkey inferotemporal cortex. Brain Res. 1121, 76-82,

Sato, T., Kawamura, T., I wai, E., 1980, Responsiveness of inferotemporal single units o visual pattern stimuli in monkeys performing discrimination. Exp. Brain Res.

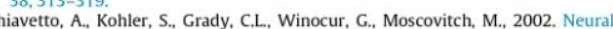
correlates of memory for object identity and object location: effects of aging . Leventhal, A.G. 1998. Signal timing across the macaque visual system. I. Neurophysiol, 79, 3272-3278

schmolesky, M.T., Wang, Y., Pu, M., Leventhal, A.G., 2000. Degradation of stimulus selectivity of visual cortical cells in senescent rhesus monkeys. Nat. Neurosci. 3

Schwartz, E.L., Desimone, R., Albright, T.D., Gross, C.G., 1983. Shape recognition and inferior temporal neurons. Proc, Natl. Acad, Sci. U SA 80, 5776-577, 209, 1255-12:56,

einberg, D.L, Logothetis, N.K., 1997. The role of temporal cortical areas in perceptual organization. Proc. Nati. Acad. Sci. U S A 94, 3408-3413.

Spear, P.D., 1993. Neural bases of visual deficits during aging. Vision Res. 3:3,

pear, P.D., Moore, R.J., Kim, C.B., Xue, J.T., Tumosa, N.. 1994. Effects of aging on the primate visual system: spatial and temporal processing by lateral geniculate

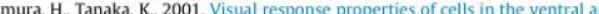
dorsal parts of the macaque inferotemporal cortex. Cereb. Cortex 11, 384-399. naka, K., Saito, H., Fukada, Y., Moriya, M., 1991. Coding visual images of objects in the inferotemporal cortex of the macaque monkey. J. Neurophysiol, 66, 170-189 Tanaka, K., 1997. Mechanisms of visual object recognition: monkey and human studies. Curr. Oplin. Nom Pobio, $7,523-529$

pson, K.G., Hanes, D.P., Bichot, N.P., Schall, J.D., 1996. Perceptual and motor

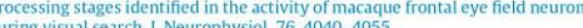
ges, J., Gordon, T.P., Mcclure, H.M. Hall, E.C., Peters, A., 1988, Survival Rate and Life-Span of Rhesus-Monkeys at the Yerkes-Regional-Primate-Research-Center: Am. J. Primatol. 15, 263-273.

Tompa, T., Sary, G., 2010. A review on the inferior temporal cortex of the macaque. Brain Res. Rev. 62, 165-182

emblay, M.E., Zettel, M.L., Ison, J.R., Allen, P.D., Majewska, A.K., 2012. Effects of ging and sensory loss on glial cells in mouse visual and auditory cortices. Gli

Van Impe, A., Coxon, J.P., Goble, D.J., Doumas, M., Swinnen, S.P., 2012. White matter fractional anisotropy predicts balance performance in older adults. Neurobiol. Aging 33, 1900-1912

Ungerleider, L.G., Mishkin, M.., 1982. Two cortical visual systems. In: Ingle, David, J. Goodale, Melvyn, A., Mansfield, Richard,J.W. (Eds.), Analysis of Visual Behaviour. The MIT Press, Cambridge, MA, pp. 549-580

Wang, H., Xie, X., Li, X., Chen, B., Zhou, Y., 2006. Functional degradation of visual

X

(ition to receptive field structures of monkey infentribution of CABAergic inhiCortex 12, 62-74.

Wang, Y.C., Zhou, Y.F., Ma, Y.Y., Leventhal, A.G.., 2005. Degradation of signal timing in cortical areas $\mathrm{M}$ and Wang, Y.Z., 2001. Effects of aging on shape discrimination. Optom Vis. Sci. 78

447-454.
Weymouth, A.E., McKendrick, A.M., 2012. Shape perception is altered by normal Winkler, A.M., Ridgway, G.R., Webster, M.A., Smith, S.M., Nichols, T.E., 2014, Permutation inference for the general linear model. Neuroimage 92, 381-397.

Xi, M.C., Liu, R.H., Engelhardt, J.K., Morales, F.R., Chase, M.H., 1999. Changes in the axonal conduction velocity of pyramidal tract neurons in the aged cat. Neuro-

Yang, Y., Liang, Z, Li, G., Wang, Y., Zhou, Y., Leventhal, A.G., 2008. Aging affects conrast response functions and adaptation of middle temporal visual area neurons Y of V1 and MT neurons in rhesus monkeys. Brain Res. 1274, 21-27.

Yang, Y..Z Zhang, J., Liang, Z., Li, G.., Wang, Y., Ma, Y.., Zhou, Y.., Leventhal, A.G., 2009 Aging affects the neural representation of speed in Macaque area MT. Cereb.

Yu, S., Wang, Y., Li, X., Zhou, Y., Leventhal, A.G., 2006. Functional degradation of extrastriate visual cortex in senescent rhesus monkeys. Neuroscience 140
$1023-1029$. 
III. 
Research Report

\title{
An investigation of the white matter microstructure in motion detection using diffusion MRI
}

\author{
Gergö Csete $e^{a, 1}$, Nikoletta Szabóa, ${ }^{a, b, 1}$, Alice Rokszinc, Eszter Tóth ${ }^{a}$ \\ Gábor Braunitzer', György Benedek ${ }^{c}$, László Vécsei ${ }^{a, d}$, \\ Zsigmond Tamás Kincses ${ }^{a, b, *}$ \\ aDepartment of Neurology, Faculty of Medicine, Albert Szent-Györgyi Clinical Centre, \\ University of Szeged, Szeged, Hungary \\ bInternational Clinical Research Centre, St. Anne's University Hospital, Brno, Czech Republic \\ ${ }^{\mathrm{C} D e p a r t m e n t}$ of Physiology, Faculty of Medicine, University of Szeged, Szeged, Hungary \\ ${ }^{\mathrm{d}}$ MTA-SZTE Neuroscience Research Group, Semmelweis u. 6, H-6725 Szeged, Hungary
}

\section{A R T I C L E I N F O}

Article history:

Accepted 4 May 2014

Available online 14 May 2014

\section{Keywords:}

Motion detection

Vision

White matter microstructure

DTI

MRI

\begin{abstract}
A B S T R A C T
One of the most widely investigated functions of the brain is vision. Whereas special attention is often paid to motion detection and its modulation by attention, comparatively still little is known about the structural background of this function. We therefore, examined the white matter microstructural background of coherent motion detection. A random-dot kinematogram paradigm was used to measure the sensitivity of healthy individuals' to movement coherence. The potential correlation was investigated between the motion detection threshold and the white matter microstructure as measured by high angular resolution diffusion MRI. The Track Based Spatial Statistics method was used to address this correlation and probabilistic tractography to reveal the connection between identified regions. A significant positive correlation was found between the behavioural data and the local fractional anisotropy in the posterior part of the right superior frontal gyrus, the right juxta-cortical superior parietal lobule, the left parietal white matter, the left superior temporal gyrus and the left optic radiation. Probabilistic tractography identified pathways that are highly similar to the segregated attention networks, which have a crucial role in the paradigm. This study draws attention to the structural determinant of a behavioural function.
\end{abstract}

"Correspondence to: Neuroimaging Research Group, Department of Neurology, Albert Szent-Györgyi Clinical Centre, University of Szeged, Semmelweis u. 6, H-6725 Szeged, Hungary.

E-mail address: kincses.zsigmond.tamas@med.u-szeged.hu (Z.T. Kincses).

URL: http://www.nepsy.szote.u-szeged.hu/ kincsesz (Z.T. Kincses).

${ }^{1}$ These authors contributed equally.

http://dx.doi.org/10.1016/j.brainres.2014.05.006

0006-8993/o 2014 Elsevier B.V. All rights reserved. 


\section{Introduction}

The neural substrates of motion sensitivity are located at different levels of the visual system. Electrophysiological investigations have revealed that in the retina subgroups of ganglion cells react differently to moving lights some responding with activation and others with decreased firing (Sivyer et al., 2010) Similarly, the lateral geniculate nucleus (LGN) (Stanley et al. 2012), the striate cortex (Beckett et al., 2012) and some peri- and parastriate areas (Sary et al., 1995; Larsson et al., 2010) respond to a dot moving across the receptive fields of the neurons. The higher levels of perception, such as global motion recognition seems to be located in the middle temporal area (MT), media superior temporal cortex (MST), and the fundus of the superio temporal cortex (FST), that are similar in monkeys and humans (Morrone et al., 2000), and was suggested to make up a complex: V5/MT+ (Boussaoud et al., 1990; Morrone et al., 2000). This area receives direct input from the primary visual cortex (V1) (Maunsell and van Essen, 1983; Felleman and Van Essen, 1991), LGN (Sincich et al., 2004), and also extrastriate regions, such as V2 (Lewis and Van Essen, 2000). These neurons with a broadly binocular representation and a relatively large receptive field $(\sim 15-20)$, (Angelucci et al., 2002), have a principal role in motion and directional sensitivity (Chawla et al., 1998).

Motion transparency (e.g. two motions superimposed on each other) as applied in the form of a random-dot kinematogram is frequently used to identify motion-sensitive cortical regions in functional imaging (Friston et al., 1997) and electrophysiological experiments (Antal et al., 2005; Braunitzer et al. 2012). It affords an opportunity for the analysis of integrated action vision in close-to-real situations. Moreover, through modulation of the attention to the various components of the task the mechanism by which attentional networks interacts with motion detection areas can be revealed (Buchel et al. 1998; Kellermann et al., 2012).

Although functional imaging experiments offer a unique opportunity for the examination of functional activation in various behavioural conditions, it was recently suggested that behavioural performance is also strongly influenced by the underlying brain structure. It was shown that the correlation between the individual structural variability and the behavioura performance can identify the neuroanatomical structures involved (Maguire et al., 2000). Moreover, if white matter structure-related parameters are investigated, this approach provides a unique opportunity for the identification of the white matter tracts associated with the certain functions (Floel et al.
2009). Such parameters can be defined by diffusion tensor imaging that may address the integrity of the white matter microstructure. The relationship between the locally measured diffusion parameters and the behavioural data is capable of revealing the coupling of structure and function (Johansen-Berg, 2010). Human studies have implied that short-term visuo-motor learning enhances the fractional anisotropy in the cortico-spinal tract (Landi et al., 2011). Plastic brain changes were found in a sequence-learning task as a correlation between the local FA and steeper leaming curves (Tomassini et al., 2011). Recent combined imaging and histological studies demonstrated that spatial learning and motor learning result in locally enhanced myelination (Blumenfeld-Katzir et al., 2011; Sampaio-Baptista et al., 2013), which is reflected in enhanced FA in the diffusion MRI

Most of the available information on the V5/MT+ stems from monkey experiments, human fMRI and positron emission tomography (PET) studies. Such knowledge is mostly functional in nature, but the structural background of motion detection is not sufficiently well known, especially that regarding the white matter pathways. In the current study, we set out to identify the white matter structural background of motion detection. We investigated the correlation between the white matter diffusion parameters and the intersubject variability for the threshold of detection for coherent motion in a random-dot kinematogram paradigm.

\section{Results}

2.1. Correlation of the white matter integrity and the motion detection threshold

The mean motion detection threshold was $18.87 \pm 5.79 \%$ coherent motions. A significant positive correlation was found between the motion detection threshold and the local FA in the right frontal cortex in the posterior part of the right superior frontal gyrus $(p<0.0032$, non-corrected), the right juxta-cortical superior parietal lobule $(p<0.0032$, non-corrected), the left parietal white matter $(p<0.001$, non-corrected), the left superior temporal gyrus $(p<0.0026$, non-corrected) and the left optic radiation $(p<0.0036$, non-corrected) (Table 1, Figs. 2 and 3$)$.

\subsection{Connectivity of the white matter region having} correlated microstructure with behavioural data

The right frontal cluster showed strong connections to the identical region in the left hemisphere and to the right

\section{Table 1 - Regions correlating with the white matter structural integrity.}

\begin{tabular}{|c|c|c|c|c|c|c|}
\hline Anatomical structure & Side & $\begin{array}{l}\text { Size } \\
\text { (voxel) }\end{array}$ & $\begin{array}{l}\text { Peak } p \\
\text { value }\end{array}$ & $\begin{array}{l}x \\
(\mathrm{~mm})\end{array}$ & $\begin{array}{l}y \\
(\mathrm{~mm})\end{array}$ & $\begin{array}{l}\mathrm{z} \\
(\mathrm{mm})\end{array}$ \\
\hline $\begin{array}{l}\text { Deep intraparietal white matter under the parieto-occipito-temporal } \\
\text { junction }\end{array}$ & Left & 21 & 0.001 & -42 & -48 & 29 \\
\hline Juxtacortical white matter in the superior temporal gyrus & Left & 16 & 0.0026 & -51 & -33 & 8 \\
\hline Juxtacortical superior parietal lobule & Right & 6 & 0.0032 & 36 & -64 & 32 \\
\hline $\begin{array}{l}\text { Juxtacorical white matter under the posterior part of the right superior } \\
\text { frontal gyrus }\end{array}$ & Right & 4 & 0.0032 & 16 & -15 & 58 \\
\hline White matter in the occipital pole & Left & 4 & 0.0036 & -18 & -86 & 12 \\
\hline
\end{tabular}


cortico-spinal tract, and a smaller pathway leading to the right parietal lobe (Figs. 2 and 4). The right superior parietal cluster exhibited the strongest connectivity to the right superior temporal gyrus. The cluster in the left parietal white matter had strong connections to the angular gyrus, the frontal white matter (through the putative superior longitudinal fascicle) and the occipito-temporal junction. The cluster in the superior temporal gyrus had connections to

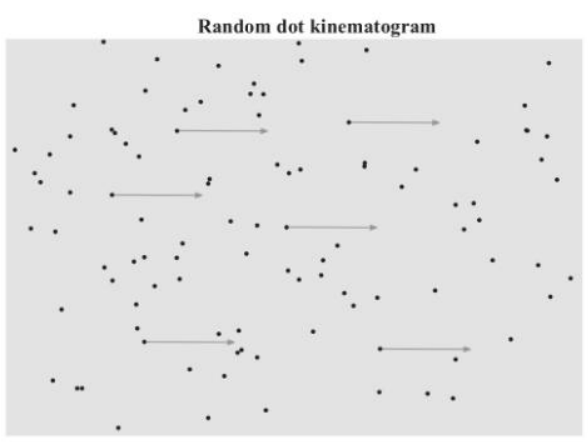

Fig. 1 - Random dot kinetogram. A static illustration of the stimulus. In each trial a given percentage of the dots moved coherently to the right or to the left (targets indicated by the arrows), while the rest moved randomly (noise). Here a $6 \%$ condition is shown with a total of 100 dots, 6 out of which belong to the coherently moving group (arrows). the angular gyrus, which led further to the frontal cortex through the arcuate fascicle, and a smaller fibre tract connected the cluster to the parietal white matter under the precuneus (not shown)

\section{Discussion}

This study has revealed local correlations between the white matter microstructure measured by diffusion MRI and the coherent motion detection performance, in several regions that can be linked to motion detection and attention functions. Our findings demonstrated that there is a substantial correlation between the white matter microstructure and function.

Earlier electrophysiological (Dubner and Zeki, 1971), lesion (Zihl et al., 1983) and imaging studies (Zeki et al., 1991: Tootel et al., 1995) identified motion-sensitive visual areas along the occipital-temporal-parietal axis, which is also known as the dorsal visual stream. Within these, probably the most important is the V5/MT+ complex, which has been shown to express robust activation in tasks involving moving stimuli. These results led us to presume the possibility of covariation between the motion detection threshold and the local microstructure along the white matter pathways connecting the motion-sensitive visual areas. Surprisingly, but not inexplicably, none of the white matter regions identified in our analysis were along the pathway running from the primary visual areas to V5/MT+. This might be due to a highly-tuned visual processing system.

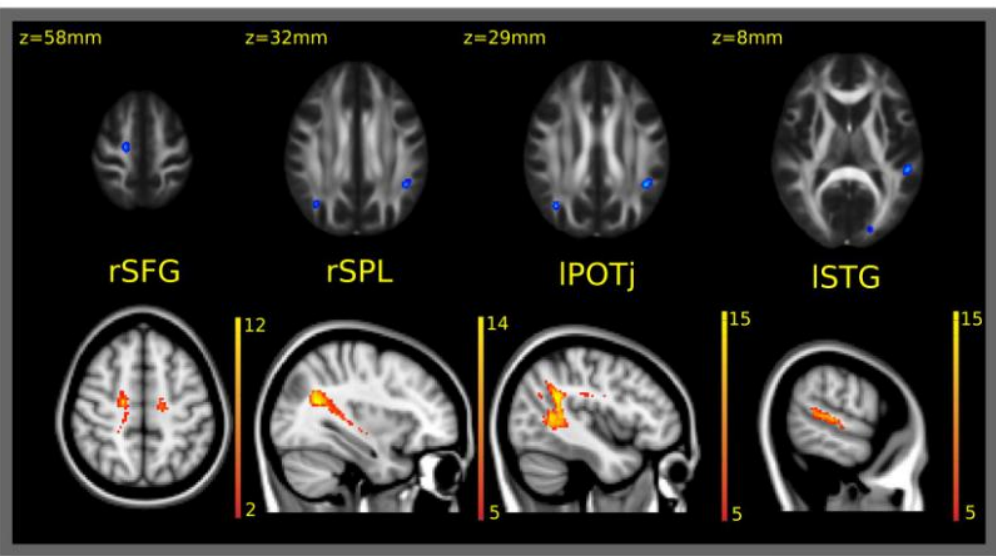

Fig. 2 - Correlation of the white matter microstructure with the motion detection threshold and the connectivity of regions. The upper row shows regions of significant correlation between voxel-wise fractional anisotropy and the motion detection threshold ( $p<0.01$, uncorrected; clusters are thickened for better visualisation). Statistical images are overlaid on the FMRIB58 standard brain. The lower row indicates the connectivity of the regions in the upper row. Labels (rSFG: right superior frontal gyrus; rSPL: right superior parietal lobe; IPOTj: left parieto-occipito-temporal junction; 1STG: left superior temporal gyrus) indicate the seed regions of the tractographies depicted in the lower row. Individual probabilistic tractography is thresholded at 500 particles, binarised and summed across subjects in MNI space. Various thresholds are chosen for each tractography results for better visualisation. 

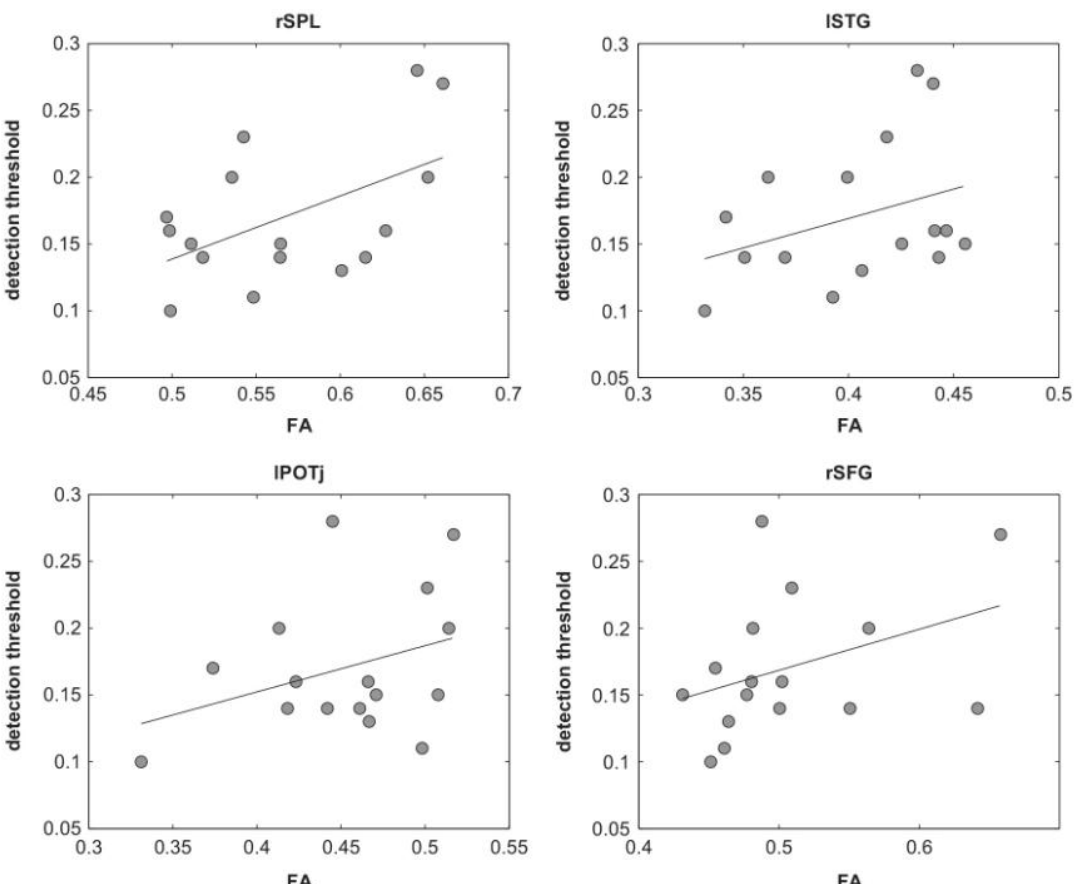

Fig. 3 - Correlation of the cluster mean fractional anisotropy and the coherent motion detection threshold. rSFG: right superior frontal gyrus; rSPL: right superior parietal lobe; IPOTj: left parieto-occipito-temporal junction; and lSTG: left superior temporal gyrus.

However, there was a strong correlation between the motion detection threshold and the microstructure of the white matter pathways connected to regions apparently identical to those with showing attention-related activity. Such a correlation seems feasible, since the detection of a coherent motion in a random noise is a highly attentiondemanding task, and hence a better performance can be expected in individuals with stronger attentional modulation Extrastriate areas (and mainly V5/MT+) have substantial attentional modulation from attention networks (Buche et al., 1998; Kellermann et al., 2012). Fundamental components of these networks are located in the frontal and parietal lobes. In a seminal investigation in which a similar randomdot kinematogram was used, areas related to attention to visual motion were identified (Buchel et al., 1998). The identified areas in the frontal eye field, the premotor cortex and the posterior parietal cortex demonstrated a high level of correspondence with the cortical regions to which the white matter regions projected in our analysis. A framework was earlier proposed for two segregated attentional systems: the dorsal attention network, responsible for goal-directed (top-down) attentional modulation, which includes parts of the dorsal parietal cortex (the superior parietal lobule and the intraparietal sulcus) and the superior frontal cortex (near or at the frontal eye field - FEF) and the ventral network driven by salient stimuli (bottom-up modulation), including the temporo-parietal junction and the inferior frontal cortex (Corbetta and Shulman, 2002). A recent investigation revealed structural correlates of such a separated attention system (Yin et al., 2012). The bottom-up orienting function proved to be negatively correlated with the cortical thickness in the angular gyrus and the superior parietal lobule and the mean diffusivity under the inferior parietal lobe. In contrast, the alerting, putatively top-down attention function was correlated with the FA in the superior frontal region (the white matter under the frontal-eye field, similarly as found in our analysis). Interestingly, our analysis revealed a correlation with motion detection threshold in white matter tracts that overlap with both the dorsal and ventral attention systems. This is not surprising, since continuous attention is directed to the moving dot pattern during the experiment and the ventral salience system might also be activated when the coherent motion pattern is finally detected. Moreover, segregated branches of the superior longitudinal fascicle have been reported to be involved in the two attention networks (Thiebaut de Schotten et al., 2011). The cortical projection of the most dorsal branch overlaps with the dorsal attention network, and the projections of the most ventral part overlap 


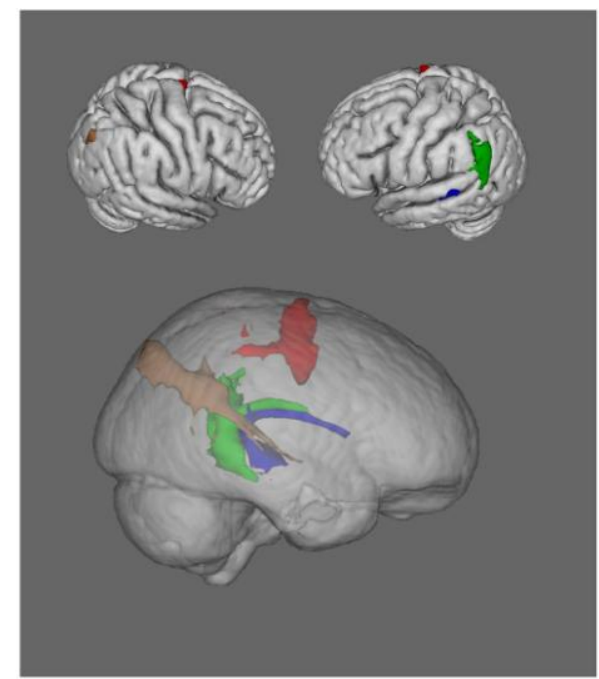

Fig. 4 - Pathways running through the white matter regions showing a significant correlation between microstructure and behavioural measures. The lower image indicates the pathways; the upper two images (right and left views) show the cortical projections. The inferior and medial branches of the superior longitudinal fascicle on the left are indicated in blue and green. The superior branch of the right superior longitudinal fascicle and part of the cortico-spinal tract originating from the putative frontal-eye field are indicated in red. The connection on the right, between the superior parietal lobule and the superior temporal gyrus, is indicated in brown.

with the ventral attention network. In contrast, the projection of a third branch of the superior longitudinal fascicle partially overlaps with the dorsal and the ventral attention network. This structural link might suggest a communication between the two systems, with redirection of the goal-directed attention mediated by the dorsal network to the events identified as salient by the ventral network (Corbetta and Shulman, 2002; Thiebaut de Schotten et al., 2011). Recent findings (Gruber et al., 2010) and an integrative theory of mind (AbuAkel and Shamay-Tsoory, 2011) suggest the importance of the interaction in both systems, where the temporo-parietal junction plays an important role of connection and shifting of mental states and orientation. It was recently hypothesied (Caclin et al., 2012) that the V5/MT+ response depends on the dorsal/ventral interactions. Further evidence indicated the involvement of the temporo-parietal junction in both attentional networks (Chica et al., 2011).

Previous imaging and electrophysiological studies found that the activity of V5/MT + is strongly modulated by attention (Treue and Maunsell, 1996; O'Craven et al., 1997; Buchel et al., 1998). Functional and effective connectivity analyses suggested that the attentional modulation arises from modulation of the connectivity strength within a network containing visual, parietal and frontal regions (Buchel and Friston, 1997; Marreiros et al., 2008). Functional connectivity studies have considered the possible site of attentional modulation (Buchel and Friston, 1997; Friston et al., 1997; Penny et al., 2004). A two-state dynamic causal modelling analysis suggested that modulation of the forward connections from V1 to V5 are crucial in attention to visual motion (Marreiros et al., 2008). Our results seem somewhat contradictory. The performance in the attention-demanding motion detection task correlated with the integrity of the white matter tracts within the attention networks rather than with those pathways connecting the attention networks to the visual regions or connecting various visual areas. This could suggest that the bottom line of the performance in the motion detection task is the "structural integrity" of the attentional networks.

Functional imaging is the classical approach through which to identify the structures behind certain brain functions. Thanks to refined imaging and analysis methods, it recently became possible to correlate variations in brain structure with variations in behavioural measures (Johansen-Berg, 2009, 2010). The relevance of white matter diffusion properties in certain behavioural performances is not yet clear, but nerve diameter and myelination, which relate to the microstructure measurable by DTI, can relate to the conduction velocity and the dispersion of the neuronal signal (Hodgkin and Huxley, 1952). Along these lines, it was earlier shown that a greater BOLD response in the visua cortex correlated with higher FA in the optic radiation (Toosy et al., 2004). The better the bimanual coordination, the higher FA detectable in the corpus callosum (Johansen-Berg et al., 2007). A training-induced FA increase has been detected in the intraparietal sulcus (Scholz et al., 2009).

Our study is certainly not without limitations. The correlation of the white matter structural connectivity and the functional and effective connectivity needs further investigation. Additionally, modulation of the attentional component of the motion detection task may reveal further characteristics of the network. Furthermore, the TBSS approach restricts the analysis to the core of the white matter fibre bundles, in order to eliminate the errors arising from mis sregistration, but neglects the periphery of the tracts (Smith et al., 2006).

We hope that our results may facilitate the understanding of visual perception and the mechanism of motion detection, and that this will help to place the pathomechanism of neglect and other attention-related disorders into perspective.

\section{Experimental procedures}

\subsection{Subjects}

Sixteen healthy volunteer subjects (average age was 26.5 (range: $21-40$ years); 9 female, 11 right-handed, mean body mass index $=22.05 \pm 4.22$ ) participated in the study. None of them suffered from any neurological or psychiatric diseases. All subjects had normal or corrected-to-normal (20/20) visual acuity. 


\subsection{Ethics}

All study participants provided their written informed consent in accordance with the Declaration of Helsinki; the study was approved by the ethics committee at the University of Szeged (Ref. no.: 87/2009).

\subsection{Psychophysical test}

The motion detection threshold was measured with random-dot kinematogram (Fig. 1). Stimuli were generated with Psychophysics Toolbox Version 3 (http://psychtoolbox. org/), under MatLab (MathWorks, Inc.) on a PC, and presented on a 24-in. LCD monitor at a resolution of 1920 by 1200 pixels and at a $60 \mathrm{~Hz}$ refresh rate. The stimuli were 100 moving black dots in random positions with variable coherence rates. Stimuli were presented on a neutral grey background in a rectangular stimulation field occupying $60 \%$ of the whole screen. Subjects were seated with the face at $0.5 \mathrm{~m}$ from the screen and the stimulation field subtended an area of 35.74 by 22.34 visual angles. The diameter of each dot was 10 pixels $(\sim 3 \mathrm{~mm})$. In each trial, a given percentage of the dots moved coherently to the right or to the left, while the remainder moved in random directions. After each trial, movementstarting points were regenerated. One trial lasted approximately $0.8 \mathrm{~s}$ ( 50 consecutive frames), during which each dot travelled $38.4 \mathrm{~mm}$. (For more details see: (Braunitzer et al. 2012)). The task of the subjects was to indicate whether the coherently moving dots moved to the left or to the right by pressing the appropriate cursor button on the keyboard. The absolute coherence threshold was determined by the QUEST adaptive threshold-seeking algorithm (Watson and Pelli, 1983).

\subsection{Data acquisition}

Neuroimaging data acquisitions were carried out on a 1.5 GE Signa Excite HDxt MR Scanner (GE Healthcare, Chalfont St. Giles, UK). Three-dimensional spoiled gradient echo images (FSPGR: echo time [TE]: $4.1 \mathrm{~ms}$; repetition time [TR]: $10.276 \mathrm{~ms}$; matrix: $256 \times 256$; field of view [FOV]: $25 \mathrm{~cm} \times 25 \mathrm{~cm}$; flip angle: 15 ; in-plane resolution: $1 \mathrm{~mm} \times 1 \mathrm{~mm}$; slice thickness: $1 \mathrm{~mm})$ and 60-direction diffusion-weighted images with 6 non-diffusion-weighted reference volumes (TE: $93.8 \mathrm{~ms}$; TR: $16.000 \mathrm{~ms}$; matrix: $96 \times 96$; FOV: $23 \mathrm{~cm} \times 23 \mathrm{~cm}$; flip angle: $90^{\circ}$; in-plane resolution: $2.4 \mathrm{~mm} \times 2.4 \mathrm{~mm}$; slice thickness: $2.4 \mathrm{~mm} ; b: 1000 \mathrm{~s} / \mathrm{mm}^{2}$; number of excitations [NEX]: 2; array spatial sensitivity encoding technique [ASSET]) were acquired for all subjects.

\subsection{Data analysis}

4.5.1. Correlation of diffusion parameters with behavioural measures

Diffusion data were corrected for eddy currents and movement artefacts by 12 degree of freedom affine linear registration to the first non-diffusion-weighted reference image. An algorithm included in the FMRIB Diffusion Toolbox (FDT) of FSL (v.4.0) fitted diffusion tensors at each voxel (Smith et al., 2004). The FA was computed for the whole brain. In order to reduce the possible errors arising from misalignment of the images, we used the Tract Based Spatial Statistics (TBSS) method (Smith et al., 2007). The FA images for all subjects were aligned into a common space with the non-linear registration tool FNIRT, which uses a b-spline representation of the registration warp field. A mean FA image was created and the threshold was set at $F A=0.2$, yielding a mean $F A$ skeleton at the centres of all tracts common to the group. The aligned FA data for each subject were then projected onto this skeleton and the resulting data were fed into voxel-wise cross-subject statistics. Modelling and inference by using the standard general linear model (GLM) design set-up was accomplished by using permutation-based cluster analysis $(n=5000)$ as implemented in the FSL software package (Nichols and Holmes, 2002). The design was encoded for the motion detection threshold value. Statistical thresholding was carried out with a novel method of Threshold Free Cluster Enhancing (Smith and Nichols, 2009). Since a correlation was expected only in well-circumscribed regions of the visual and attention networks, it would have been overconservative to correct for multiple correlations based on the total number of voxels in the skeleton. Hence, we used the non-corrected statistics thresholded at the $1 \%$ significance level. Only clusters larger than 4 voxels were considered for further analysis and discussion.

\subsubsection{Structural connectivity}

Connectivity of the regions showing a significant correlation with the motion detection threshold was defined by probabilistic tractography (FDT, part of FSL: http://www.fmrib.ox. ac.uk/fsl/fdt). A multifibre diffusion model was fitted that estimates the probability distribution in the direction of 1 or more fibre populations at each voxel (Behrens et al., 2007). Probabilistic tractography was then performed from any brain voxel by tracing streamline samples through these probabilistic distributions in the fibre direction. For tractography, we generated 5000 streamline samples from each seed voxel to build up a connectivity distribution. The number of these samples passing through each brain voxel was interpreted as proportional to the probability of connection to the seed voxel. By fitting a multifibre model to our diffusion data, we were able to trace pathways through regions of fibre crossing (Behrens et al., 2007). The seed masks were the binary masks of the suprathreshold clusters of the TBSS analysis. The result of the tractography was registered to standard space, binarised and summed over subjects for visualisation.

\section{Acknowledgments}

The study was supported by the Neuroscience Research Group of the Hungarian Academy of Sciences and University of Szeged, Project FNUSA-ICRC (No. CZ.1.05/1.1.00/02.0123) from the European Regional Development Fund, an OTKA Grant (PD 104715), the Hungarian Brain Research Program (Grant no. KTIA_13_NAP-A-II/20). The publication was supported by the European Union and co-funded by the European Social Fund, (Project title: "Telemedicine-focused research activities in the field of Mathematics, Informatics 
and Medical Sciences" Project number: TÁMOP-4.2.2.A-11/1/ KONV-2012-0073). This research was supported by the European Union and the State of Hungary, co-financed by the European Social Fund in the framework of TÁMOP 4.2.4. A/2-11-1-2012-0001 'National Excellence Program'.

R E F E R E N C E S

Abu-Akel, A., Shamay-Tsoory, S., 2011. Neuroanatomical and neurochemical bases of theory of mind. Neuropsychologia 49, 2971-2984.

Angelucci, A., Levitt, J.B., Walton, E.J., Hupe, J.M., Bullier, J., Lund, J.S., 2002. Circuits for local and global signal integration in primary visual cortex. J. Neurosci. 22, 8633-8646.

Antal, A., Temme, J., Nitsche, M.A., Varga, E.T., Lang, N., Paulus, W., 2005. Altered motion perception in migraineurs: evidence for interictal cortical hyperexcitability. Cephalalgia: Int. J Headache 25, 788-794.

Beckett, A., Peirce, J.W., Sanchez-Panchuelo, R.M., Francis, S. Schluppeck, D., 2012. Contribution of large scale biases in decoding of direction-of-motion from high-resolution fMRI data in human early visual cortex. NeuroImage 63, 1623-1632.

Behrens, T.E., Berg, H.J., Jbabdi, S., Rushworth, M.F.

Woolrich, M.W., 2007. Probabilistic diffusion tractography with multiple fibre orientations: what can we gain?. Neurolmage 34, 144-155.

Blumenfeld-Katzir, T., Pasternak, O., Dagan, M., Assaf, Y., 2011. Diffusion MRI of structural brain plasticity induced by learning and memory task. PloS One 6, e20678.

Boussaoud, D., Ungerleider, L.G., Desimone, R., 1990. Pathways for motion analysis: cortical connections of the medial superior temporal and fundus of the superior temporal visual areas in the macaque. J. Comp. Neurol. 296, 462-495.

Braunitzer, G., Rokszin, A., Kobor, J., Benedek, G., Nagy, A. Kincses, Z.T., 2012. Delayed development of visual motion processing in childhood migraine. Cephalalgia 32, 492-496.

Buchel, C. Friston, KJ., 1997. Modulation of connectivity in visual pathways by attention: cortical interactions evaluated with structural equation modelling and fMRI. Cereb. Cortex (New York, NY) 7, 768-778.

Buchel, C., Josephs, O., Rees, G., Turner, R., Frith, C.D., Friston, K.J., 1998. The functional anatomy of attention to visual motion. A functional MRI study. Brain: J. Neurol. 121 (Pt 7), 1281-1294.

Caclin, A., Paradis, A.L., Lamirel, C., Thirion, B., Artiges, E., Poline, J.B., Lorenceau, J., 2012. Perceptual alternations between unbound moving contours and bound shape motion engage a ventral/dorsal interplay. J. Vis. 12.

Chawla, D., Phillips, J., Buechel, C., Edwards, R., Friston, K.J., 1998. Speed-dependent motion-sensitive responses in V5: an fMRI study. NeuroImage 7, 86-96.

Chica, A.B., Bartolomeo, P., Valero-Cabre, A., 2011. Dorsal and ventral parietal contributions to spatial orienting in the human brain. J. Neurosci.: Off. J. Soc. Neurosci. 31, 8143-8149.

Corbetta, M., Shulman, G.L., 2002. Control of goal-directed and stimulus-driven attention in the brain. Nat. Rev. Neurosci. 3, 201-215.

Dubner, R., Zeki, S.M., 1971. Response properties and receptive fields of cells in an anatomically defined region of the superior temporal sulcus in the monkey. Brain Res. 35, 528-532.

Felleman, D.J., Van Essen, D.C., 1991. Distributed hierarchica processing in the primate cerebral cortex. Cereb. Cortex 1 , 1-47.

Floel, A., de Vries, M.H., Scholz, J., Breitenstein, C., Johansen-Berg, H., 2009. White matter integrity in the vicinity of Broca's area predicts grammar learning success. Neurolmage 47 , predicts gram
Friston, K.J., Buechel, C., Fink, G.R., Morris, J., Rolls, E., Dolan, R.J., 1997. Psychophysiological and modulatory interactions in neuroimaging. Neurolmage 6, 218-229.

Gruber, O., Diekhof, E.K., Kirchenbauer, L., Goschke, T., 2010. A neural system for evaluating the behavioural relevance of salient events outside the current focus of attention. Brain Res. 1351, 212-221.

Hodgkin, A.L., Huxley, A.F., 1952. Propagation of electrical signals along giant nerve fibers. Proc. R. Soc. Lond. B Biol. Sci. 140, 177-183

Johansen-Berg, H., 2009. Imaging the relationship between structure, function and behaviour in the human brain. Brain Struct. Funct. 213, 499-500.

Johansen-Berg, H., 2010. Behavioural relevance of variation in white matter microstructure. Curr. Opin. Neurol. 23, 351-358.

ohansen-Berg, H., Della-Maggiore, V., Behrens, T.E., Smith, S.M. Paus, T., 2007. Integrity of white matter in the corpus callosum correlates with bimanual co-ordination skills. Neurolmage 36 (Suppl 2), S16-S21.

Kellermann, T., Regenbogen, C., De Vos, M., Mossnang, C., Finkelmeyer, A., Habel, U., 2012. Effective connectivity of the human cerebellum during visual attention. J. Neurosci. 32. 11453-11460.

Landi, S.M., Baguear, F., Della-Maggiore, V., 2011. One week of motor adaptation induces structural changes in primary motor cortex that predict long-term memory one year later. J. Neurosci. 31, 11808-11813.

Larsson, J., Heeger, D.J., Landy, M.S., 2010. Orientation selectivity of motion-boundary responses in human visual cortex. J. Neurophysiol. 104, 2940-2950.

Lewis, J.W., Van Essen, D.C., 2000. Corticocortical connections of visual, sensorimotor, and multimodal processing areas in the parietal lobe of the macaque monkey. J. Comp. Neurol. 428 , 112-137.

Maguire, E.A., Gadian, D.G., Johnsrude, I.S., Good, C.D., Ashburner, J., Frackowiak, R.S., Frith, C.D., 2000. Navigation-related structural change in the hippocampi of taxi drivers. Proc. Natl. Acad. Sci. USA 97, 4398-4403.

Marreiros, A.C., Kiebel, S.J., Friston, K.J., 2008. Dynamic causal modelling for fMRI: a two-state model. Neurolmage 39, 269-278. Maunsell, J.H., van Essen, D.C., 1983. The connections of the middle temporal visual area (MT) and their relationship to a cortical hierarchy in the macaque monkey. I. Neurosci. 3, 2563-2586.

Morrone, M.C., Tosetti, M., Montanaro, D., Fiorentini, A., Cioni, G. Burr, D.C., 2000. A cortical area that responds specifically to optic flow, revealed by fMRI. Nat. Neurosci. 3, 1322-1328.

Nichols, T.E., Holmes, A.P., 2002. Nonparametric permutation tests for functional neuroimaging: a primer with examples. Hum. Brain Mapp. 15, 1-25.

O'Craven, K.M., Rosen, B.R., Kwong, K.K., Treisman, A., Savoy, R.L., 1997. Voluntary attention modulates fMRI activity in human MT-MST. Neuron 18, 591-598.

Penny, W.D., Stephan, K.E., Mechelli, A., Friston, K.J., 2004 Comparing dynamic causal models. Neurolmage 22. 1157-1172

Sampaio-Baptista, C., Khrapitchev, A.A., Foxley, S., Schlagheck, T. Scholz, J., Jbabdi, S., DeLuca, G.C., Miller, K.L., Taylor, A., Thomas, N., Kleim, J., Sibson, N.R., Bannerman, D., JohansenBerg, H., 2013. Motor skill learning induces changes in white matter microstructure and myelination. J. Neurosci.: Off. J. Soc. Neurosci. 33, 19499-19503.

Sary, G., Vogels, R., Kovacs, G., Orban, G.A., 1995. Responses of monkey inferior temporal neurons to luminance-, motion-, and texture-defined gratings. J. Neurophysiol. 73, 1341-1354.

Scholz, J., Klein, M.C., Behrens, T.E., Johansen-Berg, H., 2009. Training induces changes in white-matter architecture. Nat. Neurosci. 12, 1370-1371. 
Sincich, L.C., Park, K.F., Wohlgemuth, M.J., Horton, J.C., 2004 Bypassing V1: a direct geniculate input to area MT. Nat. Neurosci. 7, 1123-1128

Sivyer, B., van Wyk, M., Vaney, D.I., Taylor, W.R., 2010. Synaptic inputs and timing underlying the velocity tuning of directionselective ganglion cells in rabbit retina. J. Physiol. 588 , 3243-3253.

Smith, S.M., Jenkinson, M., Johansen-Berg, H., Rueckert, D. Nichols, T.E., Mackay, C.E., Watkins, K.E., Ciccarelli, O., Cader, M.Z., Matthews, P.M., Behrens, T.E., 2006. Tract-based spatial statistics: voxelwise analysis of multi-subject diffusion data. Neurolmage $31,1487-1505$

Smith, S.M., Jenkinson, M., Woolrich, M.W., Beckmann, C.F. Behrens, T.E., Johansen-Berg, H., Bannister, P.R., De Luca, M. Drobnjak, L., Flitney, D. ., Niazy, R.K Saunders, J. Vickers, J. Zhang, Y. De Stefano, N. Brady, J.M., Matthews, P. 2004 Chang, Y, De Stefano, N., Brady, JM, Matthews, PM, 2004. Advances in functional and structural MR image analysis and implementation as FSL. Neurolmage 23 (Suppl 1), S208-S219.

Smith, S.M., Johansen-Berg, H., Jenkinson, M., Rueckert, D., Nichols, T.E., Miller, K.L., Robson, M.D., Jones, D.K., Klein, J.C. Bartsch, A.J., Behrens, T.E., 2007. Acquisition and voxelwise analysis of multi-subject diffusion data with tract-based spatial statistics. Nat. Protoc. 2, 499-503.

Smith, S.M., Nichols, T.E., 2009. Threshold-free cluster enhancement addressing problems of smoothing, threshold dependence and localisation in cluster inference. NeuroImage 44, 83-98.

Stanley, G.B., Jin, J., Wang, Y., Desbordes, G., Wang, Q., Black, M.J. Alonso, J.M., 2012. Visual orientation and directional selectivity through thalamic synchrony. J. Neurosci. 32 , 9073-9088.

Thiebaut de Schotten, M., Dell'Acqua, F., Forkel, S.J., Simmons, A. Vergani, F., Murphy, D.G., Catani, M., 2011. A lateralized brain network for visuospatial attention. Nat. Neurosci. 14, 1245-1246.

Tomassini, V., Jbabdi, S., Kincses, Z.T., Bosnell, R., Douaud, G. Pozzilli, C., Matthews, P.M., Johansen-Berg, H., 2011. Structural and functional bases for individual differences in motor learning. Hum. Brain Mapp. 32, 494-508.

Toosy, A.T., Ciccarelli, O., Parker, G.J., Wheeler-Kingshott, C.A., Miller, D.H., Thompson, A.J., 2004. Characterizing functionstructure relationships in the human visual system with functional MRI and diffusion tensor imaging. NeuroImage 21, 1452-1463.

Tootell, R.B., Reppas, J.B., Dale, A.M., Look, R.B., Sereno, M.I., Malach, R., Brady, T.., Rosen, B.R., 1995. Visual motion aftereffect in human cortical area MT revealed by functional magnetic resonance imaging. Nature 375, 139-141.

Treue, S., Maunsell, J.H., 1996. Attentional modulation of visual motion processing in cortical areas MT and MST. Nature 382 , 539-541.

Watson, A.B., Pelli, D.G., 1983. QUEST: a Bayesian adaptive psychometric method. Percept. Psychophys. 33, 113-120.

Yin, X., Zhao, L., Xu, J., Evans, A.C., Fan, L., Ge, H., Tang, Y., Khundrakpam, B., Wang, J., Liu, S., 2012. Anatomical substrates of the alerting, orienting and executive control components of attention: focus on the posterior parietal lobe. Plos One 7, e50590.

Zeki, S., Watson, J.D., Lueck, C.J., Friston, K.J., Kennard, C. Frackowiak, R.S., 1991. A direct demonstration of functional specialization in human visual cortex. J. Neurosci. 11, 641-649.

Zihl, J., von Cramon, D., Mai, N., 1983. Selective disturbance of movement vision after bilateral brain damage. Brain: J. Neurol. 106 (Pt 2), 313-340. 